\title{
Coherent Control in the PRESENCE OF Disorder
}


Samenstelling promotiecommissie:

Prof. dr. J.L. Herek

Prof. dr. L. Kuipers

Prof. dr. H.J. Bakker

Prof. dr. K.-J. Boller

Dr. R. van Gastel

Prof. dr. W. Pfeiffer

Prof. dr. W.L. Vos
Universiteit Twente, Enschede, Nederland

Universiteit Twente, Enschede, Nederland

FOM Instituut Amolf, Amsterdam, Nederland

Universiteit Twente, Enschede, Nederland

Universiteit Twente, Enschede, Nederland

Universitat Bielefeld, Bielefeld, Duitsland

Universiteit Twente, Enschede, Nederland

Copyright (c) 2010 by P. van der Walle

The work described in this thesis was performed at the FOM-Institute for Atomic and Molecular Physics (AMOLF), Amsterdam, The Netherlands, and at the MESA+ Institute for Nanotechnology, University of Twente, Enschede, The Netherlands. The work is part of the research programme of the Stichting Fundamenteel Onderzoek der Materie (FOM), which is financially supported by the Nederlandse Organisatie voor Wetenschappelijk Onderzoek (NWO).

All rights reserved. No part of the material protected by this copyright notice may be reproduced or utilized in any form or by any means, electronic or mechanical, including photocopying, recording or by any information storage and retrieval system, without the prior permission of the author.

ISBN 978-90-365-2991-4 


\title{
COHERENT CONTROL IN THE PRESENCE OF DISORDER
}

\author{
PROEFSCHRIFT
}

ter verkrijging van

de graad van doctor aan de Universiteit Twente, op gezag van de rector magnificus, prof. dr. H. Brinksma

volgens besluit van het College voor Promoties

in het openbaar te verdedigen

op woensdag 17 maart 2010 om 16.45 uur

door

Peter van der Walle

geboren op 3 oktober 1979

te Haifa, Israel 
Dit proefschrift is goedgekeurd door de promotoren:

Prof. dr. J.L. Herek

Prof. dr. L. Kuipers 


\section{CONTENTS}

1 Introduction $\quad 7$

1.1 Coherent control . . . . . . . . . . . . 8

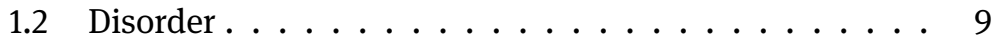

2 Experimental 13

2.1 Open and closed loop coherent control . . . . . . . . . 14

2.2 Laser system . . . . . . . . . . . . . . . . . . . . . . . . . . . . . . .

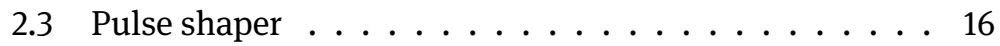

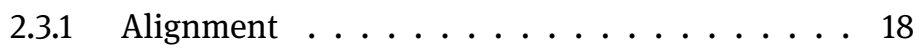

2.3.2 Calibration ............... 19

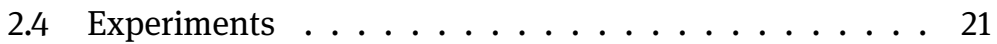

2.4 .1 Samples .................. 21

2.4 .2 Microscope ............... 23

2.4 .3 Pump-probe ................ 24

2.5 Covariance Matrix Adaptation algorithm . . . . . . . 25

3 Phase shaped Second-Harmonic Generation 29

3.1 Introduction ................ 30

3.2 Optimization of second harmonic generation . . . . 31

3.3 Resonant second harmonic generation ........ 33

3.3.1 Interferometric autocorrelation . . . . . . . 35

3.3.2 Chirp scans ............... 36

3.3.3 Homogeneous and Inhomogeneous line widths . 39

3.4 Shaping the SHG fitness landscape . . . . . . . . . 42

3.4.1 Constructing a basis set . . . . . . . . . 44

3.4 .2 Conclusion ............... 46

4 Simulation of Light on Planar Metal Nanostructures 47

4.1 Planar metal nanostructures . . . . . . . . . 48

4.2 Simulation method . . . . . . . . . . . 48

4.3 Small metal particles . . . . . . . . . . . . 50

4.4 Percolation structures . . . . . . . . . . . . . . . . 52

4.4.1 Spatial phase correlation ........ 52 
4.4.2 chirp scan on a percolation structure . . . . . 55

5 Phase Control over Local Field Intensities 57

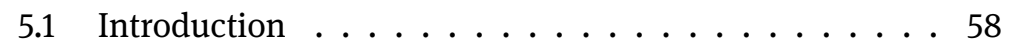

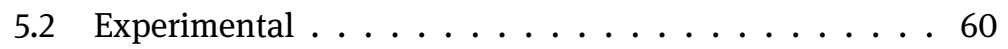

5.3 Results .................. 62

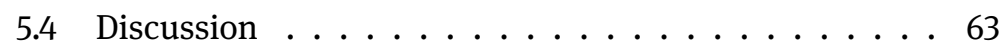

6 Second-Harmonic Generation on Disordered Hole Arrays 67

6.1 Introduction ................. 68

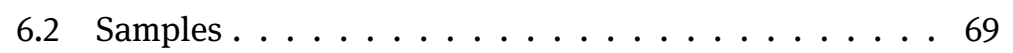

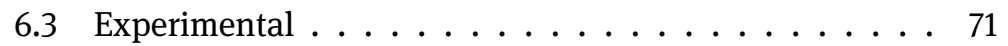

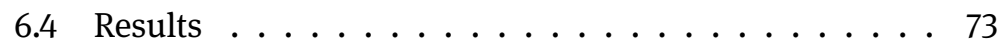

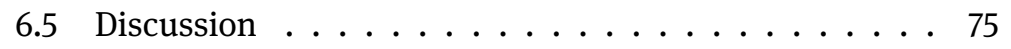

7 Quantum Control Spectroscopy of Solvent-induced Decoherence $\quad 77$

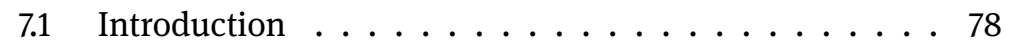

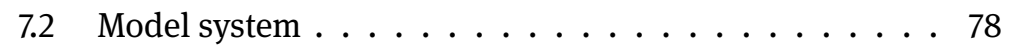

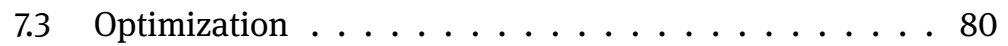

7.4 Results .................... 82

7.4.1 Potential mechanism . . . . . . . . 82

7.4 .2 Solvent dependence . . . . . . . . . . . . 86

7.5 Conclusion ...................... 90

$\begin{array}{ll}\text { Bibliography } & 93\end{array}$

$\begin{array}{ll}\text { Summary } & 103\end{array}$

$\begin{array}{ll}\text { Samenvatting } & 105\end{array}$

$\begin{array}{ll}\text { Acknowledgements } & 107\end{array}$ 


\section{Chapter 1 \\ INTRODUCTION}

In this Chapter the concepts of coherent control and disorder are briefly introduced. We illustrate how these concepts are related to the work described in this Thesis. Finally, an outline of the Thesis is given. 


\subsection{Coherent control}

Coherent control is the control of a system by light, making use of a coherent process. In a coherent process, there is a well defined phase relation between the input and output. When multiple pathways exist, the efficiency of the process is determined by the relative phase of the pathways. If all the pathways are in phase, the process is enhanced.

The simplest form of coherent control uses just two laser beams ${ }^{1}$, one at frequency $\omega_{0}$ and one at $3 \omega_{0}$. An atomic or molecular transition can be excited via two pathways, by absorbing 3 photons from the first laser and by absorbing one photon from the second laser. The transition can be excited selectively by choosing the relative phase between the laser beams. If the lasers are in phase, the transition is excited, and with the lasers out of phase, the transition is not excited. For reasons of symmetry, this scheme does not work for a laser at $2 \omega_{0}$, as this laser can't excite to the same state as the laser at $\omega_{0}$.

Larger molecules have a densely packed vibrational manifold. To cover all these vibrational states, light at many frequencies is required. For the control of such molecules, pulsed lasers with a large bandwidth are often used. The relative phases of the frequencies are set using a pulse shaper. The inner workings of a pulse shaper are described in Chapter 2. Here, we will focus on the concept of a shaped pulse.

A short laser pulse has a large spectral bandwidth. Initially the relative phases of the frequency components are all zero, producing the shortest possible pulse, the transform-limited (TL) pulse (Figure 1.1 left). The frequencies in the laser spectrum all arrive at the same time in a TL pulse, similar to striking all the keys on a piano simultaneously. A straightforward modification to the pulse shape is analogous to the musical scale sweep, in which the instanteneous frequency changes linearly with time (Figure 1.1 center). A pulse with this shape is called a chirped pulse.

Chirped pulses play an important role in this thesis. Theory of chirp scans is developed in Chapter 3 where we derive how resonant properties of samples can be extracted by measuring a non-linear response with pulses that have a range of different chirps. Such scans of the chirp are performed in the experiments of Chapters 5 and 6. Finally, in Chapter 7 a non-linearly chirped pulse is found to be optimal in the control of a dye in solution.

Many systems can be studied by performing open-loop scans with simple pulse shapes, but some control problems require a more 


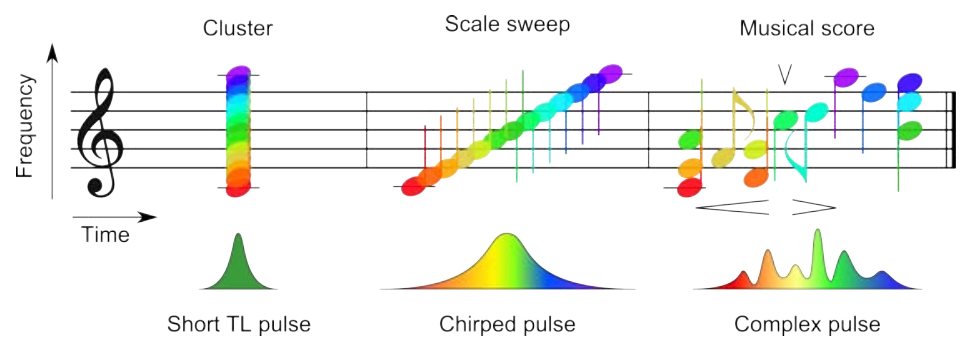

Figure 1.1: Pulse shaping is the optical analog of composing music. The available 'notes' are the frequency components in the spectrum of the laser. These components can be arbitrarily spread in time by adjusting the settings on the pulse shaper. One of the simplest shaped pulses is a chirped pulse in which the instantaneous frequency is swept in time.

sophisticated pulse shape. An example of such a complex pulse is the optical melody in Figure 1.1 on the right. In this pulse shape, the spectral phase is a complicated function of the laser frequency. For most problems that require such a complex pulse shape, the solution is not known in advance. Finding the solution is a difficult task that requires a trail-and-error approach. This approach is often implemented in a closed feedback loop, in which a learning algorithm iteratively improves the pulse shape ${ }^{2,3}$. The details of this approach can be found in Chapter 2 .

\subsection{Disorder}

Any material is built-up from atoms. The relative positions of the atoms determine the structure of the material. When the atoms are neatly stacked in rows, columns and layers, a crystal structure is formed. Many properties of a material are caused by such an arrangement, for example the formation of electron bands ${ }^{4}$, distinct spots in an X-ray diffraction pattern ${ }^{4}$ or a polarization dependent refractive index for light ${ }^{5}$.

Crystals can also be made by arranging larger building blocks, such as large molecules, spheres or even holes, in an ordered structure. These crystals are sometimes referred to as meta-materials, because much larger building blocks are treated like atoms. The size of the building blocks should be matched to the physics that one wishes to influence with the material. Electrons and X-ray radiation with a short wavelength can interact with atoms, while visible light has a 


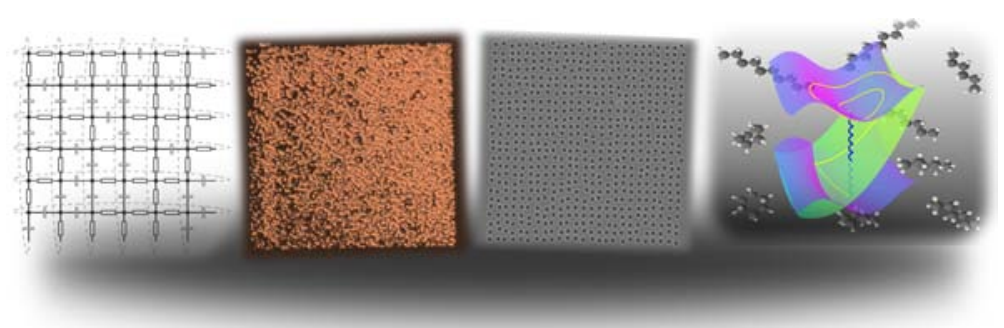

Figure 1.2: The disordered samples that are described in this thesis. From left to right, a random resistor/capacitor network, a film of randomly positioned gold islands, a thick gold film with square holes on a lattice with disorder and a dye molecule in solution that has constantly changing energy levels due to interactions with the solvent.

wavelength much larger than an atom and therefore does not 'feel' individual atoms. Thus, a crystal for visible light requires larger building blocks and can for example be formed with small glass spheres with a size on the order of the wavelength of the light, a so-called opal.

Disorder is introduced when a perfect crystal structure is disturbed. The structure can be disturbed by small random changes in the positions, orientations or sizes of the building blocks. This disorder weakens or even eliminates the effects of the crystal structure. In the extreme case, disorder can also introduce new effects such as Anderson localization ${ }^{6}$. This effect causes electrons or photons to become localized in loops that keep an electron or photon trapped by scattering.

As disorder is caused by random changes to the large number of atoms in a crystal, the crystal can be disturbed in countless different ways. It would be very tedious to calculate the properties of a disordered structure by including the exact positions of all the atoms. Luckily, to capture the important physics the exact realization of the disorder is not relevant, only the average relative positions of the atoms are required as input. These positions can be described with the structure function or more commonly a correlation function that is its Fourier transform ${ }^{7}$. An ensemble averaged correlation function that describes a crystal structure takes the form:

$$
C_{n n}\left(x_{1}, x_{2}\right)=\left\langle n\left(x_{1}\right) n\left(x_{2}\right)\right\rangle,
$$

where the function $n(x)$ is a delta-function whenever the position $x$ is the position of a building block of the material. 
We applied pulse shaping to a few different disordered systems (Figure 1.2): an electrical network of randomly placed resistors and capacitors, two types of gold nanostructures and to a laser dye in solution. The presence of disorder is easily visible for the electrical network and the two nanostructure samples. The network serves as a model system for a film of small islands of gold randomly positioned on a glass slide (Chapter 5). The islands are completely disordered, as the correlation function decays within a distance the size of a single island. The disorder in the random gold islands makes to local field as a response to light different at each position on the surface (Chapter 4). We can use the difference is local response by tailoring the illuminating light to the response at a specific position and selectively excite it.

The other nanostructure structure is a smooth gold film with very small holes milled through the film. The holes were initially placed in an ordered array, but we added random displacements to the positions of each individual hole. The resulting structures are similar to a melting crystal (see Figure 6.2). The light transmission through a hole array relies on the coupling between the holes. In an ordered array, the coupling is very efficient for some specific wavelengths. We have introduced disorder in the positions of the holes to disturb this coupling and see how the light transmission is effected (Chapter 6).

In Chapter 7 we describe the study of a laser dye in solution. The disorder in this sample is in the solvent surrounding the dye. At roomtemperature energy is present in the low-frequency modes of the solvent molecules. This energy causes the molecules to vibrate. The vibrations surrounding the dye molecule cause the transition energy, required to excite the dye to its excited state, to vary with time. These fluctuations of energy, or transition frequency, are often described with a frequency-correlation function ${ }^{8}$.

The variation of the energy levels by the solvent, disturbs the coherence in the system required for coherent control. By repeating a control experiment in different solvents we show that the amount in which the coherence is lost can be varied and has direct consequences for the ability to coherently control the dye $\mathrm{e}^{9,10}$. 


\section{Chapter 2 \\ EXPERIMENTAL}

This chapter is a collection of the technical details behind the experiments described in the rest of this thesis. We will follow a feedback loop that is often employed in coherent control experiments and describe its components. The pulse shaper including the laser system, the samples and methods of fabrication, the measurement techniques and the details of the used evolutionary algorithm are discussed. 


\subsection{Open and closed loop coherent control}

In coherent control there is a distinction between open-loop and closed-loop control. In an open-loop experiment one or a set of pulse shapes is tried to control a system and the results are interpreted by the experimenter. In this way, one can quickly gain new insights into a system. The disadvantage is that already something has to be know about the system to be able to choose pulse shapes that will show an effect. For many complex system this knowledge is not available, leaving only the route of closed-loop control. In this method an algorithm is used that iteratively improves the pulse shape to reach a desired goal. While the closed-loop approach result in a considerable degree of control with many systems, interpreting the optimal pulse shape and deriving the mechanism of control are difficult.

A closed loop learning experiment begins at the algorithm, where a set of random pulse shapes is generated (Figure 2.1). These pulse shapes are tested one by one with the pulse shaper. Then an experiment is performed with each of the pulse shapes and a value that we wish to optimize is measured. These values, called the fitness, are fed back to the algorithm, which modifies the set of pulse shapes based on their measured fitnesses. The loop continues until it converges on the optimal pulse shape that maximizes the fitness.

In our closed-loop experiments we have used an advanced evolutionary learning algorithm, the Covariance Matrix Adaptation (CMA) ${ }^{11}$. This algorithm is able to detect covariances in parameters that it uses, which can greatly speed up an optimization. Another important aspect is noise. The algorithm uses a measured fitness that has an uncertainty associated with it. We found that when certain settings are used, the CMA is very robust to this uncertainty ${ }^{12}$. The detail of the algorithm can be found in Section 2.5.

The main difference between open and closed-loop experiments is the application of an algorithm. Therefore, we introduce the experimental details of both types of experiments by following the loop of a closed-loop optimization in the remainder of this chapter.

\subsection{Laser system}

We start following the loop in Figure 2.1 at the pulse shaper. Before we can shape a pulse, we first have to generate one. A complicated laser system generates short pulses with a high intensity. The center 


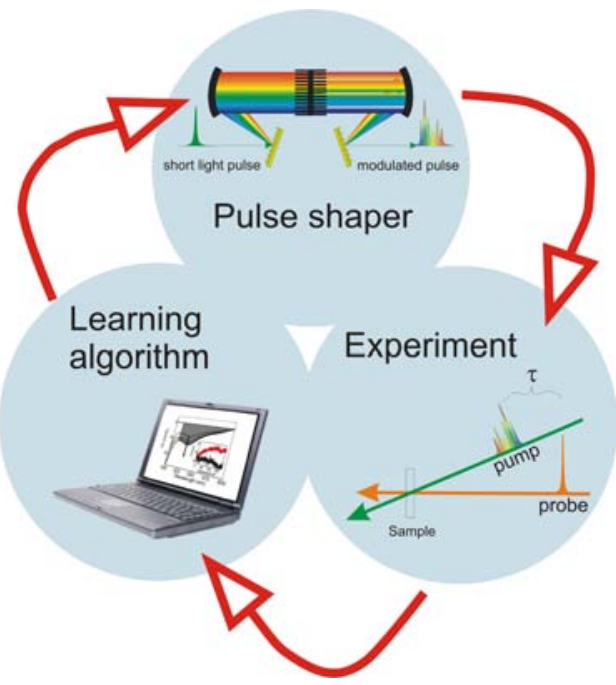

Figure 2.1: A closed-loop optimization incorporating a search algorithm that adjusts the settings on a pulse shaper, based on feedback received from an experiment. By iteratively going through the loop, the best settings (pulse shape) are found that guide the experimental system to a desired state.

wavelength is tuned in a non-linear optical process.

The experiments in Chapter 5 and 7 were performed using the same laser system. The fundamental laser source is a Titanium:Sapphire regenerative amplifier (Clark-MXR, CPA-2001). This amplifier takes seed pulses from a fiber laser that are frequency doubled and stretched by chirping, before being feed into the amplification cavity. The amplifier is pumped by a Q-switched and frequency doubled Nd:YAG laser. The YAG is pumped by a flash-lamp that is immersed in cooling water. The pumping by a flash-lamp imposes some limits on the system regarding stability, but because the amplifier is driven into saturation, pulse to pulse energy fluctuation of $.5 \%$ are typically achieved. After amplification, the pulse is passed through a grating compressor, which results in a $150 \mathrm{fs}$ long pulse containing $850 \mu \mathrm{J}$ of energy. These pulses are referred to as the 'fundamental' laser pulses.

The fundamental pulses of $150 \mathrm{fs}$ are still relatively long for ultrafast spectroscopy and pulse shaping. A non-collinear optical parametric amplifier (NOPA) is used to broaden the spectrum and thus shorten the potential pulse length ${ }^{13}$. The NOPA is pumped with a portion of the fundamental light $(250 \mu \mathrm{J})$. Of this pump beam a small part is split off and focussed into a sapphire plate. The high intensity focus in the 
sapphire produces a white light continuum, which is used as a seed to start the amplification process.

The remainder of the fundamental in the NOPA is doubled and used to pump the parametric amplification process. The white-light seed and doubled fundamental are overlapped in a BBO crystal. By tuning the delay between the two beams and the angle of the crystal, the output frequency is determined. The tuning range of the NOPA starts from $475 \mathrm{~nm}$ (limited by absorption of the idler in the BBO crystals) to $1200 \mathrm{~nm}$ (limited by the extent of the white-light continuum). The NOPA typically produces an output spectrum of $30 \mathrm{~nm}$ wide, which results in sub $20 \mathrm{fs}$ pulses in the green to around $30 \mathrm{fs}$ pulses when using the red part of the spectrum. The parametric process is more efficient in the green, but pulse energies of 10 to $20 \mu \mathrm{J}$ with a stability of $1 \%$ can routinely be achieved across the tuning range.

In the experiment described in Chapter 5, only the NOPA beam was used, whereas the experiment in Chapter 7 required a pump-probe configuration ${ }^{14}$. In this experiment, the short NOPA pulses were used as the pump and the probe was a white-light continuum generated from some of the remaining fundamental pulse.

In the experiment described in Chapter 6 we used a much simpler laser system. Only a Titanium:Sapphire oscillator was used (Coherent Micra). This laser generates short pulses ( $\sim 20 \mathrm{fs}$ ) with a center wavelength around $800 \mathrm{~nm}$. The repetition frequency is $80 \mathrm{Mhz}$, but the pulse energy is much lower than with the amplified system (average power $\sim 450 \mathrm{~mW}$ ).

\subsection{Pulse shaper}

The generated femtosecond laser pulses are too short to be effectively modulated in the time domain. However, the short pulse duration is associated with a broad spectrum, such that the pulses can be shaped to high resolution in the frequency domain ${ }^{15}$. The individual frequency components of a pulse are modulated with a specific amplitude and phase to tailor the shape of the output pulse in the time domain. A short input pulse can be shaped into a much longer pulse, only limited by the resolution of the modulation in the frequency domain. The resolution is mainly determined by the number of pixels in the used spatial light modulator (SLM). In our experiments, we used an SLM with 640 pixels across the spectrum, resulting in a shaping window of about 6 ps. This shaping window is much longer than the dephasing time of 


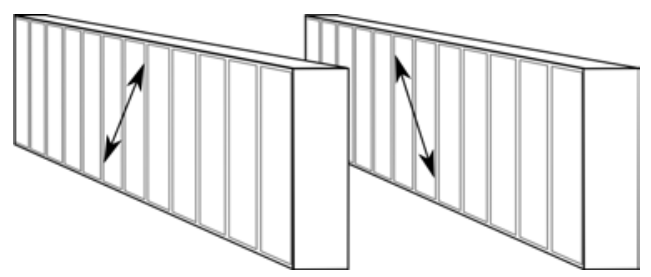

Figure 2.2: The SLM contains two masks that have the liquid crystals aligned perpendicular to each other, both at $45^{\circ}$ with the polarization of the incoming light. This orientation allows the phase and amplitude of the transmitted light to be controlled independently.

the processes we are interested in (molecular electronic dephasing and plasmon dephasing are at most a few hundreds of femtoseconds long), thus the SLM gives more than sufficient resolution.

The SLM contains two liquid crystal masks, called Master and Slave, that allow independent control of the phase and amplitude of the transmitted light (Figure 2.2). The input and output polarization of the shaper should be parallel to the plane of the table, which is ensured by placing linear polarizers on both sides of the mask. The two masks have the liquid crystals aligned at $\pm 45^{\circ}$ to the input polarization. Because of this alignment, we can consider the transmission through the masks as two independent channels (one for $-45^{\circ}$ and one for $+45^{\circ}$ ) with adjustable phase delay. The input field is divided over the channels, such that each carries half the intensity. The two channels are added together and interfere at the exit polarizer (see equation (2.1)).

$$
\begin{aligned}
E_{\text {out }}= & \frac{E_{\text {in }}}{2}\left(\mathrm{e}^{i \phi_{1}}+\mathrm{e}^{i \phi_{2}}\right) \\
= & \frac{E_{\text {in }}}{2}\left[\left(\cos \left(\phi_{1}\right)+\cos \left(\phi_{2}\right)\right)+i\left(\sin \left(\phi_{1}\right)+\sin \left(\phi_{2}\right)\right)\right] \\
= & E_{\text {in }}\left[\cos \left(\frac{\phi_{1}+\phi_{2}}{2}\right) \cos \left(\frac{\phi_{1}-\phi_{2}}{2}\right)+\right. \\
& \left.+i \sin \left(\frac{\phi_{1}+\phi_{2}}{2}\right) \cos \left(\frac{\phi_{1}-\phi_{2}}{2}\right)\right] \\
= & E_{\text {in }} \cos \left(\frac{\phi_{1}-\phi_{2}}{2}\right) \mathrm{e}^{i \frac{\phi_{1}+\phi_{2}}{2}}
\end{aligned}
$$




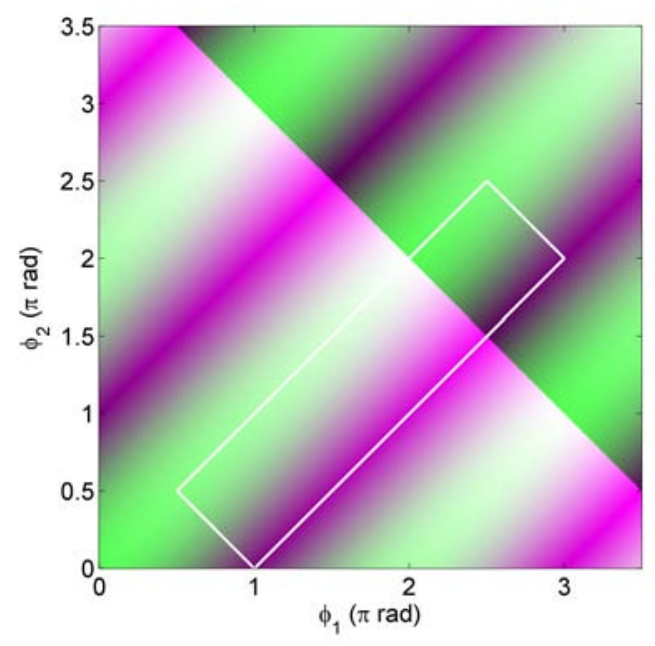

Figure 2.3: The transmission amplitude (green $0-1$ ) and phase (magenta $0-2 \pi$ ) of the output electric field as a result of different settings for $\phi_{1}$ and $\phi_{2}$. The white rectangle indicates the area containing all possible combinations of amplitude and phase that was used in the experiments.

The amplitude of the transmitted field is controlled via the difference of the phases set on each mask and the relative phase is set by the sum of the phases. To set a certain amplitude and phase on the shaper, the two phase settings for the masks have to be chosen. Figure 2.3 shows the accessible parameter space. In the experiments only a subset of this space was used, indicated by the white rectangle.

\subsubsection{Alignment}

The shaper has to be carefully aligned to ensure that all frequency components overlap at the sample position. The alignment starts by adjusting the entrance beam to be horizontal. The grating is than put in a perfect vertical position by tuning the reflected beam (0-order) to be horizontal as well. Now the grating is rotated around the normal to the surface to make the grooves vertical. The height of all diffraction orders should be the same. The spectrum is centered by placing a wire in the middle of the shaper and looking at the output spectrum with a fibre spectrometer. The grating angle is tuned until the dip caused by the wire is in the center of the spectrum. The recombination at the second grating has to be checked. When the response of the eye is uniform 
Fourier plane

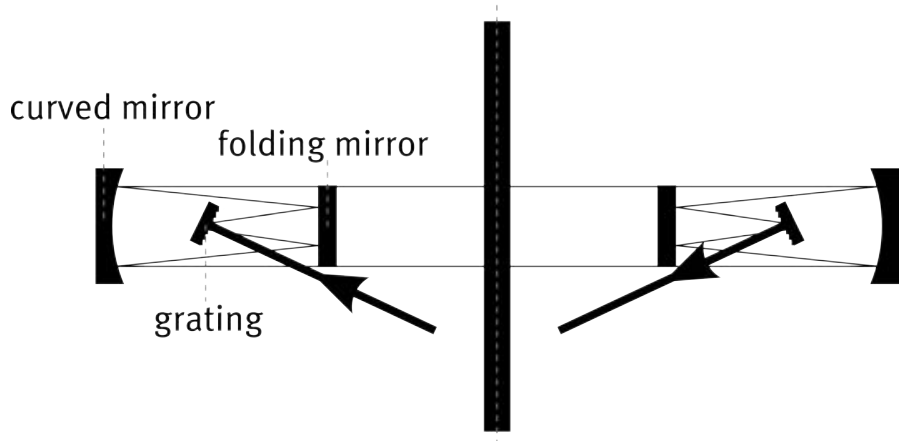

Figure 2.4: Folded arrangement of the 4-f pulse shaper. A liquid crystal mask in the Fourier-plane is used to set the phase and amplitude of individual frequency components.

across the spectrum this can be done directly. When using red to infrared wavelengths, the grating has to be imaged on a camera to get a better view of the relative intensities in the beam. An increasing part of the spectrum is blocked by sliding a card in the Fourier plane. The recombined beam should only dim and not change shape when the card is inserted. Horizontal misalignment is corrected by moving the folding mirrors to put the gratings at the focal points of the curved mirrors, while vertical misalignment is corrected by rotating the curved mirrors around the normal. Finally, recombination at a position far from the shaper is corrected by tuning the angles of the second grating. When large corrections are made in this step, it is advisable to go back to the previous step and do another iteration of adjustments.

\subsubsection{Calibration}

The calibration of the masks is done one by one. First, the master is calibrated while the effect of the slave is eliminated by writing 4095 (corresponding to $10 \mathrm{~V}$ ) to all the pixels. At this high voltage the extraordinary axis of the liquid crystals is rotated parallel to the laser beam so that the light experiences no birefringence from the mask. The transmitted light is detected on a spectrometer for spatial resolution of the response of the SLM. By writing amplitude dips at different positions on the masks and detecting where they end up on the spectrometer, the mapping from spectrometer pixels to mask pixels is determined. The 


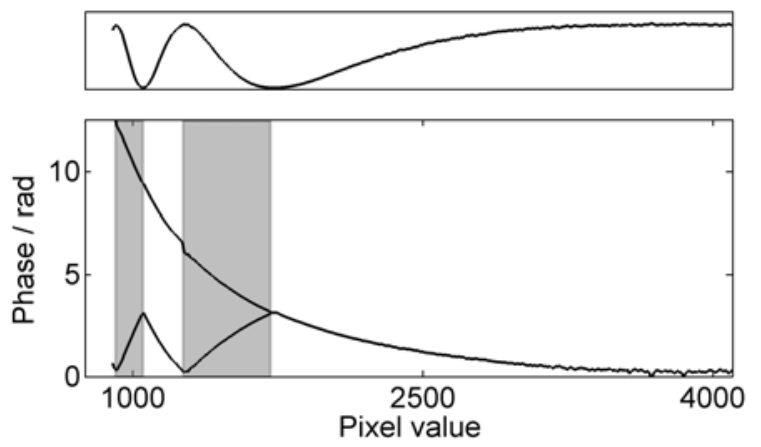

Figure 2.5: Measured transmitted intensity as function of pixel value (top) and the unwrapped trace for calibration (bottom). The pixel value corresponds to the voltage across the liquid crystals, $0-4095 \equiv 0-10 \mathrm{~V}$

voltage on the mask to be calibrated is scanned from 0 to $10 \mathrm{~V}$ and the transmission is recorded. For each pixel this scan results in a trace as shown in Figure 2.5. The shape of the trace is described by:

$$
I=I_{0} \cos ^{2}\left(\frac{\phi(V)}{2}\right)
$$

Where $\phi(V)$ is the phase retardation of the slow axis with respect to the fast axis, the birefringence, as a function of voltage. The measured trace has to be inverted to find this phase function. Because only a range of $3 \pi$ is required for full phase and amplitude shaping, we calibrate and use only the slower varying high voltage part of the liquid crystal response. The trace is inverted and unwrapped as in the bottom graph in Figure 2.5. Finally we fit the curve with a stretched exponential. The same procedure is follow to calibrate the Slave.

$$
\phi(V)=A \mathrm{e}^{-\left(\frac{V}{\tau}\right)^{\beta}}
$$

This function describes the data very well. It contains only three fitting parameters and it can be inverted analytically, so that a simple formula gives the voltage setting required for a desired phase value.

In this calibration procedure only the birefringence (i.e. the phase difference between the fast and the slow axis of the liquid crystal 
for each voltage setting) is calibrated. Variations in the path length through the mask due to a varying thickness or refractive index do not directly enter the calibration curves. These variations need to be compensated by applying a background phase function on top of the desired phase function. This background is determined by optimizing second harmonic generation of the transmitted pulse, using the CMA algorithm at the end of this chapter.

\subsection{Experiments}

Now that we are able to create shaped laser pulses, we continue the loop with an experiment. The experiment requires a sample to study and a method to see the effect of the shaped pulses on this sample is needed. The sample is either placed on a microscope to look at it directly or a probe beam is sent through the sample to measure its absorption as an effect of the shaped pulse.

\subsubsection{Samples}

Chapter 7 describes the study of a dye in solution. This dye was dissolved in its respective solvent and put into a cuvette. This cuvette was placed at the crossing point of the pump and probe beams (see Section 2.4.3).

The experiments in Chapters 5 and 6 concern gold nanostructures. The preparation of these structures requires work in a clean-room environment with nano-lithography techniques. These techniques are described below.

\section{Electron beam lithography}

To create nanostructured metal films with the shapes and sizes required, we used electron beam lithography (EBL). In this technique, an electron beam is used to write the structure in a sensitive layer. Charging of the substrate has to be prevented, as it increases the focus size of the beam and reduces resolution. Therefore, we used substrates coated with a conducting ITO layer to prevent the charging and additionally allowing for easy characterization of the samples in a scanning electron microscope after fabrication.

A bi-layer of PMMA was spin-coated on the substrate. In the area exposed to the electron beam, the long polymer chains are broken up 

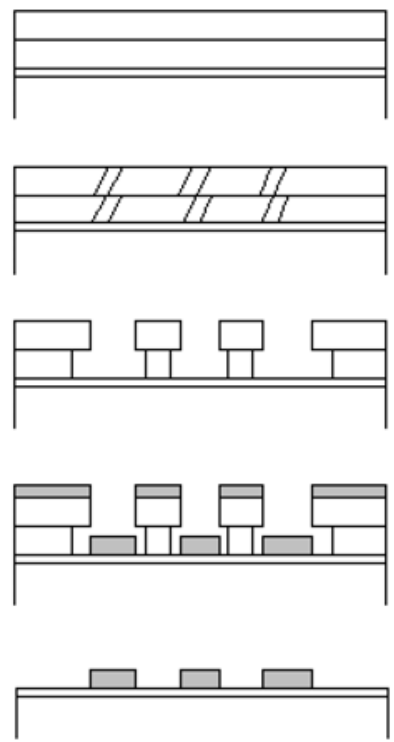

Figure 2.6: Schematic of the processing steps for electron-beam lithography. First two layers of PMMA are spin-coated on the substrate (495 KDa and 950 KDa average molecular weight). The desired structure is written by an electron beam, after which the exposed area is dissolved in a developer (MIBK). The gold is deposited on the sample and the remaining PMMA is dissolved.

into shorter chains. These short chains are soluble in the developing solution whereas long chains are not. In the developer, the exposed areas are dissolved, leaving the bare substrate.

The metal that forms the nanostructures was subsequently evaporated on to the substrate. By using a bi-layer in which the top layer consists of longer polymer chains, we prevent the nanostructures to be attached to the metal layer on top. After deposition of the metal, the residual PMMA was dissolved in a more aggressive solvent and the top metal layer pealed off, leaving only the nanostructures on the substrate.

Finally, the nanostructures were coated with quantum dots to be able to visualize the local fields on the surface ${ }^{16}$. This coating was done by dipping the substrate into a dilute solution of quantum dots in toluene and letting the solvent dry off. 


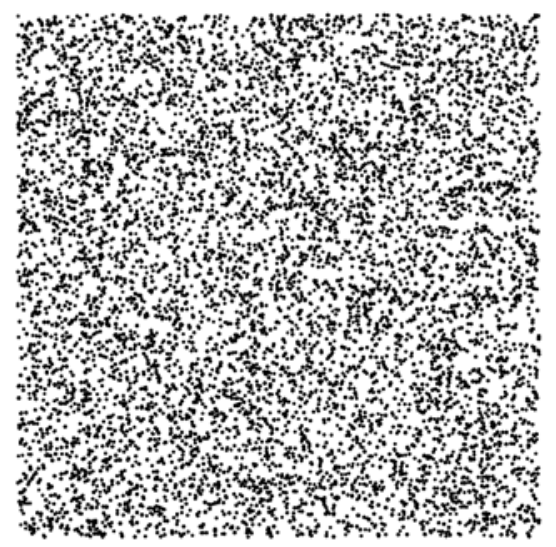

Figure 2.7: Designed random structure of gold islands.

\section{Focussed Ion-Beam Lithography}

A focused ion-beam is similar to an electron beam. Gallium ions are accelerated and focussed to a few nanometer diameter spot, which is scanned across the sample. The impact of the gallium ion is destructive to the sample. Some material is sputtered away at the focus of the beam.

Hole array samples were produced by milling the pattern into an optically thick layer of gold $(200 \mathrm{~nm})$. The controller for the ion-beam takes a stream-file that contains a list of positions and a dwell time for each position. These stream-files were directly created by a MatLab script to have full control over the milling order. A potential problem with ion-beam milling is redeposition of material. To avoid this problem the holes were created starting from the center and spiralling outwards. At the border of the holes the dose was increased in order to have a sharp definition of the edges.

\subsubsection{Microscope}

The experiment on the random gold islands was performed on a microscope. The shaped pulses illuminated the sample from the top. Here a lens with 3-axis adjustment was placed to position the beam and create a weak focus. A zero-order half-wave plate in the laser beam was used to control the polarization of the light. The angular position of this 


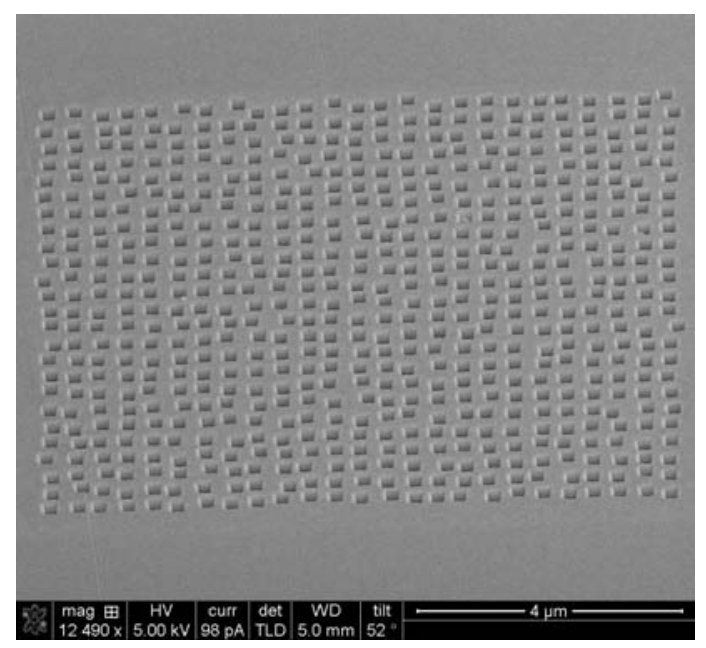

Figure 2.8: Hole array with disorder produced by Focussed Ion Beam Milling

plate was calibrated by placing a polarizer on the microscope table. The angles of the wave-plate resulting in a polarization parallel to the table-axis were determined. The fluorescence from the samples was imaged through a 1.4 NA 100x objective. An objective with this high NA allows for efficient collection of fluorescence by capturing a large solid angle of emission. After the objective filters were placed that block the transmitted laser light. Finally, an image of the fluorescence from the sample was produced on a sensitive electron-multiplying camera (EMCCD). A problem with CCDs at low light levels is the read-out noise that is added on top of the signal from each pixel. The EMCCD circumvents this problem by first amplifying the counts in each pixels, before converting the counts to a number. In this way the read-out noise relative to the signal level is reduced ${ }^{17}$.

\subsubsection{Pump-probe}

The experiments studying the effects of solvation on the control of a molecule, as described in Chapter 7, made use of a pump-probe setup $^{14}$. In this setup the sample was first excited with a shaped pump pulse and at some time later the effect of the pump pulse was probed by measuring the change in transmission with a white light probe pulse. The probe light was generated by focussing a part of the light from the CPA in a sapphire window resulting in a white light 


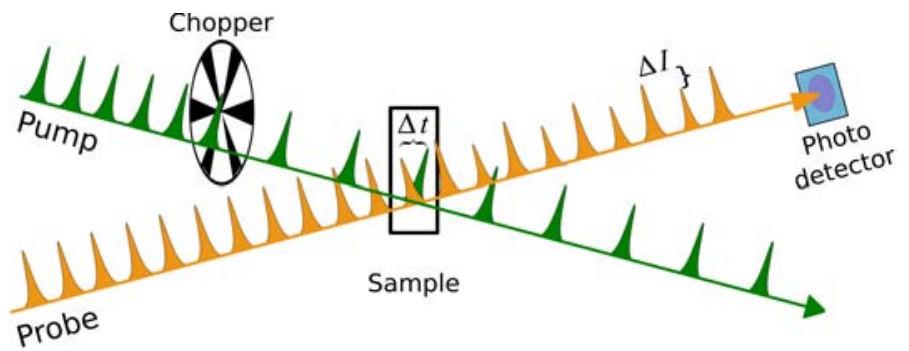

Figure 2.9: The effect of the pump pulse on the sample is measured by alternatingly measuring the transmission of the probe with the pump on and off. The dynamics of the induced absorption are determined by scanning the difference in arrival time between the two pulses.

continuum. Before the white light was generated, the CPA pulses were passed through an adjustable delay stage to be able to record the time evolution of the signal.

The transmitted probe-pulses were measured by a spectrometer with a home-built camera (DIABLO). This camera allows data acquisition of spectra at the laser repetition rate to be able to separate the spectra belonging to pump on and pump off (Figure 2.9).

\subsection{Covariance Matrix Adaptation algorithm}

The final component that closes the loop (Figure 2.1) is a search algorithm that drives an optimization. The high resolution of the pulse shaper results in a large amount of pulse shapes that can be synthesized. Especially when a problem is not well understood, finding the best pulse shape to solve it is a difficult task. We have used the Covariance Matrix Adaptation (CMA) algorithm to find the pulse shape for these complex problems ${ }^{11}$.

The CMA is an evolutionary search algorithm (Figure 2.10). Like all such algorithms, it evolves a set of trial solutions (a generation) by selectively reusing the best solutions of the previous generation (higher fitness). The CMA starts each generation from a single parent that is randomly mutated. How this mutation is performed is what defines the CMA.

While running the loop, the CMA learns which direction in the search space is more likely to yield a good solution. This direction is derived by looking at the path of successful mutations and determining the covariance matrix between these mutations. The covariance 


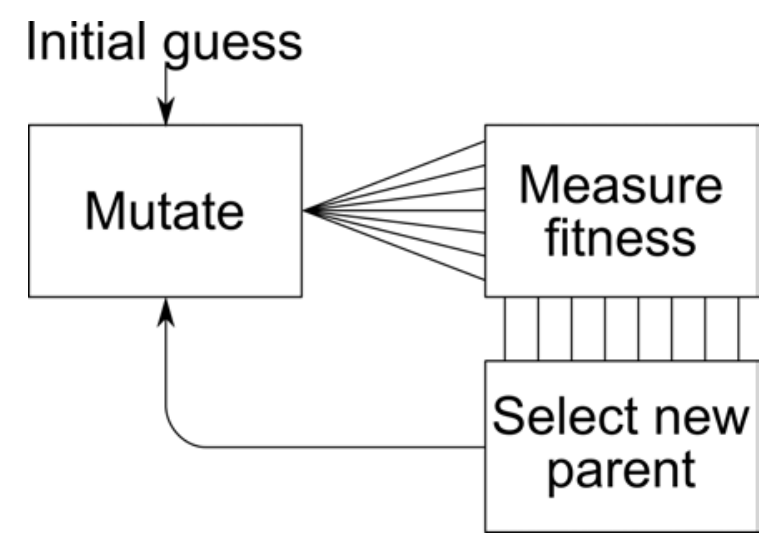

Figure 2.10: In general an evolutionary algorithm iteratively improves the fitness of the solution via selection and mutation. Specific algorithms differ in how mutation and selection are implemented.

matrix is then used to modify the probability distribution of the trial solutions (Figure 2.11).

Another important aspect of the CMA and evolutionary strategies in general is control of the step size. When the step size is too large, any learning is immediately lost. When the algorithm steps on a peak in the landscape, subsequent steps will be too far away to find this peak again and climb it. When the step size is too small, it will take many generations to climb a slope in the landscape. In this case there is learning, but it takes longer than necessary. In addition, due to the small step size, saddle points and local optima in the landscape can become traps for the algorithm.

The criteria for a too small and/or too large step size depend on the fitness landscape and on where in the landscape the algorithm is searching. Therefore, a good evolutionary strategy dynamically changes the step size to be near optimal for every phase of the search process. The CMA adapts the step size by calculating the length of the search path it has taken. In the optimal case, the steps are on average perpendicular (compare with a steepest descend method). When the step size is too small, the steps will be more or less in line and the path will be longer than a path for perpendicular steps. When the step size is too large, consecutive steps will be in opposing directions to compensate. In this case, the search path is shorter than the path with perpendicular steps. By calculating the length of the search path, the CMA is able to detect suboptimal step sizes and adjust accordingly. 


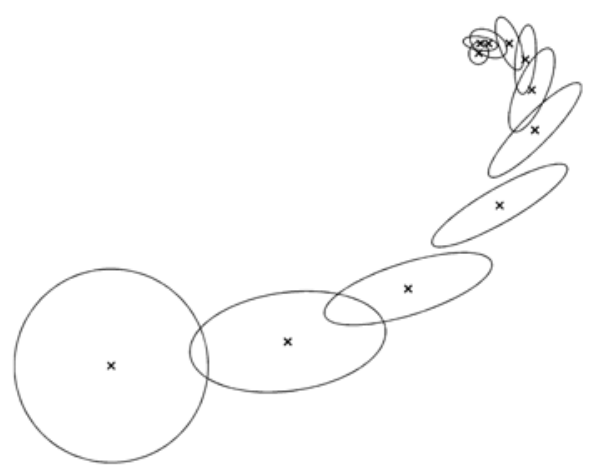

Figure 2.11: A 2-dimensional representation of how the CMA algorithm adapts the probability distribution of the trial solutions based on the direction of previous successful guesses. The ellipses depict the shape of the probability distribution in the next generation.

One caveat with step size control is that there has to be some learning. When there is no learning, the algorithm performs a random walk. The steps in this random walk are on average perpendicular and result in a search path of the same length as the path with an optimal step size. Thus, if the initial step size is chosen in a regime where there is no learning, the algorithm is unable to adjust the step size to the optimal regime. Therefore, some care has to be taken in setting the initial step size. Intuition or previous knowledge on the fitness landscape and signal-to-noise has to be used. 


\section{Chapter 3}

\section{PHASE SHAPED SECOND-HARMONIC GENERATION}

Second-harmonic generation (SHG) is one of the simplest non-linear optical effects: an electromagnetic wave interacts with a wave of the same frequency producing a wave at double the frequency. SHG with shaped pulses can be applied in many ways; the characterization of the pulses, compressing the pulses and the determination of the properties of resonances of samples. In this Chapter, we develop theory of non-resonant and resonant SHG with shaped pulses. The theory for resonant SHG is used to compare two techniques that are sensitive to resonances: the well known interferometric autocorrelation and a newly developed chirp scan. We find that the chirp scan has advantages over the autocorrelation technique that give more information on the properties of resonances.

Finally, we will look at the landscape of the SHG optimization problem when the Taylor series is used as the basis set. By visually changing the landscape into one with convex contour lines, we construct a new basis set that will give a faster and more robust convergence on the maximum of the landscape. 


\subsection{Introduction}

The frequency of optical radiation is too fast to measure directly. A measurement always yields an intensity, averaged over many cycles. The phase information of the optical wave is lost. Phase shaping an optical pulse does not change its total energy and therefore the average intensity measured on a detector is independent of the applied phase. The phase can be measured by interfering the pulse with a reference pulse. This requires the setup to maintain the length of the path of the signal and reference pulses to within a fraction of a wavelength, which is difficult when the paths are separate (as required in a pulse shaping experiment). Furthermore, to measure the absolute phase as a function of frequency, the phase of the reference pulse needs to be known.

When a reference with known phase is unavailable, a non-linear effect has to be used in which the signal is referenced with itself. Secondharmonic generation (SHG) is one of the simplest non-linear effects an electromagnetic wave interacts with a wave of the same frequency producing a wave at double the frequency. The Frequency Resolved Optical Gating (FROG) technique uses SHG with two pulse replicas for a range of delays between two pulses and spectrally resolves the generated SHG light ${ }^{18}$. If this measurement is done with sufficient resolution, the spectral phase of the input pulse can be retrieved.

At the start of our experiments, we are not interested in the exact shape of the spectral phase of the input pulse. We just need to correct the phase with the pulse shaper to be constant across the spectrum. For this purpose we use the property of SHG that it has the maximum signal for a given input spectrum, when the phase of that spectrum is constant over the spectrum. A flat spectral phase results in a (Fourier) transform-limited (TL) pulse. If we find the phase mask on the pulse shaper that produces the maximum SHG, we have found a pulse with a flat spectral phase. We add this phase mask to all the masks used in experiments and call it the background phase.

How easy it is to find the maximum SHG depends on the search landscape. This landscape is the SHG intensity as a function of the search parameters. In Section 3.4 we study how the landscape depends on the basis function used in the search and use this knowledge to construct a better basis set for the optimization of $\mathrm{SHG}^{19}$. 
In Chapter 5 we show how two-photon absorption in quantum dots can be used to probe the local field intensity on a nanostructure. When the two-photon absorption spectrum is sufficiently flat, the same mathematics as for the SHG can be applied here. In Chapter 6 we describe how the SHG from an array of holes in a metal film was studied. In this study we distorted the input pulse with a known spectral phase and measured how this distortion influenced the intensity of SHG. By analysing the data from this measurement, the position and width of a resonance on the structure was found. The theory behind this analysis is developed in this Chapter.

\subsection{Optimization of second harmonic generation}

Before the pulse shaper can be used to synthesize tailored pulse shapes, the background spectral phase of the laser pulse has to be determined and corrected. Optimization of SHG of a shaped pulse allows us to determine this phase up to an ambiguity that leads to a linear term and an offset in the spectral phase. Although our pulses are short ( $<30 \mathrm{fs}$ ), they are still several optical cycles long ( $>10$ cycles). Therefore, the offset between the carrier and the envelope of the pulse caused by the offset is not important. Similarly, a spectral phase that is linear in the frequency just shifts the arrival of the pulse in time. For practical purposes, finding a spectral phase that maximizes the SHG is sufficient to correct for the background phase of the pulse. A mathematical proof of the maximization follows below.

In addition to being applied to find the background spectral phase, the SHG optimization experiment has proven useful as a test case in the

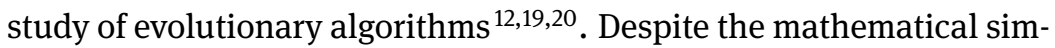
plicity of the solution, the problem of optimizing SHG has been found to be non-trivial ${ }^{21}$ and changing the parameters that an algorithm uses in its search has large effects on the learning speed of the algorithm. In Section 3.4 at the end of this chapter, we look into the optimization landscape of SHG and present parameters that result in a landscape that is easier to search through. The insights gained from these studies have led to better initial parameters for our algorithm and have enabled the optimization of more difficult problems (see Chapter 7). 
We will focus first on non-resonant SHG, which means that the nonlinear medium (e.g. a non-linear crystal) has no resonances near the fundamental and $\mathrm{SH}$ frequency.In this case the instantaneous $\mathrm{SH}$ field is directly proportional to the square of the input field,

$$
E^{(2)}(t) \propto E^{2}(t)
$$

To study the effect of the spectral phase on the SHG process, we transform equation (3.1) into the frequency domain,

$$
E^{(2)}(\omega) \propto \int_{-\infty}^{+\infty} E\left(\omega-\omega^{\prime}\right) E\left(\omega^{\prime}\right) d \omega^{\prime} .
$$

The symmetry of the process is more apparent with the change of variables $\omega^{\prime}=\frac{\omega+\omega^{\prime \prime}}{2}$, which leads to

$$
E^{(2)}(\omega) \propto \frac{1}{2} \int_{-\infty}^{+\infty} E\left(\frac{\omega-\omega^{\prime \prime}}{2}\right) E\left(\frac{\omega+\omega^{\prime \prime}}{2}\right) d \omega^{\prime \prime}
$$

The fundamental field $E(\omega)$ is a complex quantity. We can explicitly introduce the spectral phase by splitting the fields into their amplitudes and phases:

$$
\begin{aligned}
& E^{(2)}(\omega) \propto \frac{1}{2} \int_{-\infty}^{+\infty}\left|E\left(\frac{\omega-\omega^{\prime \prime}}{2}\right)\right| \mathrm{e}^{i \phi\left(\frac{\omega-\omega^{\prime \prime}}{2}\right)} \times \\
&\left|E\left(\frac{\omega+\omega^{\prime \prime}}{2}\right)\right| \mathrm{e}^{i \phi\left(\frac{\omega+\omega^{\prime \prime}}{2}\right)} d \omega^{\prime \prime} .
\end{aligned}
$$

To maximize the SHG intensity at a certain $\omega$, the phase of the SHG due to each frequency pair,

$$
\phi\left(\frac{\omega-\omega^{\prime \prime}}{2}\right)+\phi\left(\frac{\omega+\omega^{\prime \prime}}{2}\right)
$$

should be independent of $\omega^{\prime \prime}$ (see also Figure 3.1). Thus the differential of 3.5 with respect to $\omega^{\prime \prime}$ should yield zero,

$$
-\frac{1}{2} \phi^{\prime}\left(\frac{\omega-\omega^{\prime \prime}}{2}\right)+\frac{1}{2} \phi^{\prime}\left(\frac{\omega+\omega^{\prime \prime}}{2}\right)=0
$$




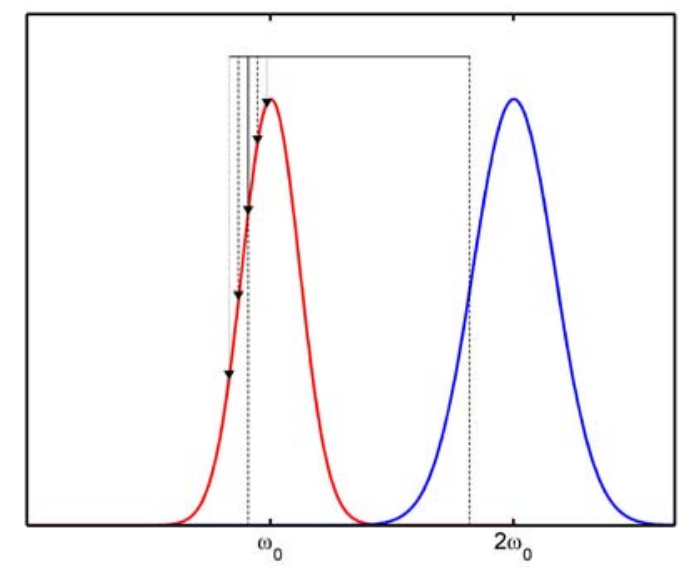

Figure 3.1: The sum frequencies of different frequency pairs in the fundamental spectrum (same line style), contribute to the same frequency in the secondharmonic spectrum. The second harmonic is maximized when all these contributions have the same phase.

When the full SHG spectrum is maximized (for all $\omega$ ), equation (3.6) can only be satisfied when the derivative of the spectral phase $\left(\phi^{\prime}(\omega)\right)$ is a constant. Any spectral phase that consists of a constant and a linear term satisfies this condition and maximizes the intensity of the SHG.

\subsection{Resonant second harmonic generation}

We will focus further on the second harmonic generated in the enhanced field of a plasmon resonance. First, we calculate the effect of the resonance on the SHG process. Then we use the result to simulate the SHG from a set of shaped pulses. Within the set, a single parameter that describes the pulse shape is scanned. Two different pulse shapes are tried, a pulse pair with variable delay and a chirped pulse with variable chirp. We discuss what details of the resonance can be extracted from such scans.

The motion of an electron as a response to the electric field of impinging light can have a resonant response. We describe this motion 


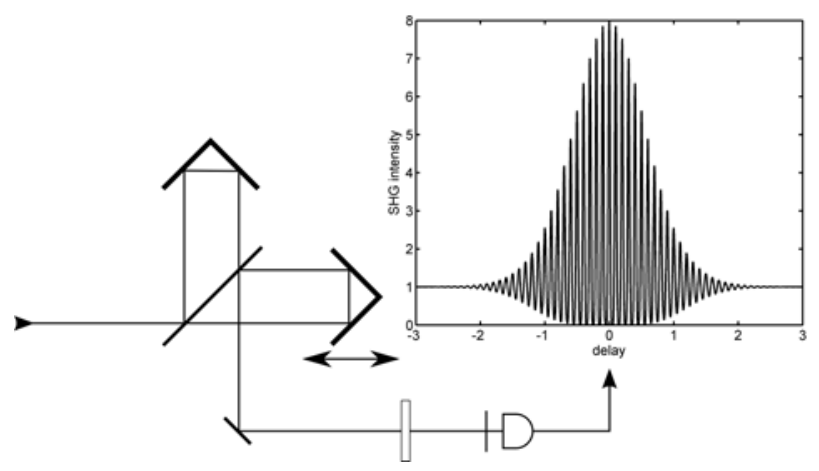

Figure 3.2: A schematic representation of a Michelson interferometer. An interferometric autocorrelation is measured by mixing two pulse replicas in a non-linear crystal. The intensity of the non-linear response is detected as a function of the delay between the two pulse replicas. The width of the trace is determined by the length of the input pulse.

by the coordinate, $x$, which obeys the following equation of motion:

$$
\ddot{x}+2 \Gamma \dot{x}+\Omega^{2} x+a x^{2}=\frac{e}{m} E(t) .
$$

In this equation, $\Omega$ is the undamped resonance frequency, $\Gamma$ is the damping rate and $a$ represents the strength of the non-linearity of the potential. The non-linear term, $a x^{2}$, can be considered a small perturbation on the linear equation. The equation can be solved by first solving the linear part,

$$
x(t)=\int_{-\infty}^{+\infty} x_{0}(\omega) E(\omega) \exp (i \omega t) d \omega
$$

with,

$$
x_{0}(\omega)=\frac{e / m}{\Omega^{2}-\omega^{2}+2 i \omega \Gamma} .
$$

This solution is then inserted into equation 3.7 and it is solved again with $-a x(t)^{2}$ as the driving term. In the following, it is assumed that only the fundamental frequency is near the resonance frequency, $\Omega$, and that the second harmonic is far from any resonance. In this case $x_{0}(\omega)$ can be simply considered as an additional modulation on the (shaped) spectrum of the input pulse, before the second harmonic field 
is generated:

$$
I_{S H G} \propto \int_{-\infty}^{+\infty}\left|\int_{-\infty}^{+\infty} x_{0}(\omega) E(\omega) \exp (i \omega t) d \omega\right|^{4} d t .
$$

Equation 3.10 is used in the following section to simulate the SHG signal for different input pulse. The effect of a resonance on an autocorrelation trace and on a chirp scan trace is calculated.

\subsubsection{Interferometric autocorrelation}

Autocorrelation is a technique to measure the length of an optical pulse $^{22}$. Two replicas of the pulse are created in a Michelson interferometer (Figure 3.2). The two pulses are overlapped inside a non-linear crystal and the amount of generated SHG is measured on a detector, while the delay between the replicas is scanned. The measured SHG intensity peaks at zero delay and the pulse length of the initial pulse is determined from the width of the measured peak. For the measurement of the pulse length, a non-resonant (i.e. instantaneous) non-linearity is required. If the response of the non-linear crystal is not constant across the bandwidth of the pulse due to a resonance in the material of the crystal, the measurement results in a longer pulse length than that of the actual pulse. This effect can be used to characterize resonances in materials by comparing the autocorrelation trace with this material as the non-linear medium with a trace measured on a non-resonant reference medium ${ }^{23}$. The difference in the measured pulse lengths is given by the lifetime of the resonance.

It was long believed that the autocorrelation technique is mostly sensitive to the homogeneous line width of the resonance and not to heterogeneous broadening ${ }^{23,24}$. It was argued that the homogeneous and heterogeneous line width of the resonance could be determined by comparing a linear extinction spectrum and the width of the resonance found with the autocorrelation ${ }^{25}$. These measurements were also supported by simulations.

Unfortunately, these simulation mistakenly included the full second-order response, both SHG and optical rectification (OR), in the calculated signal $^{26}$. In the OR process, the $-\omega$ part of the spectrum interacts with the $+\omega$ part, resulting in radiation with a low frequency. However, detectors used in SHG experiments are not sensitive to this low-frequency radiation and thus this signal is lost. 


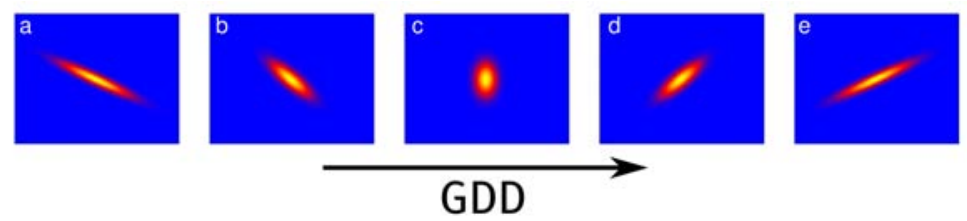

Figure 3.3: Maps of frequency versus time for several pulses with increasing group-delay dispersion (GDD increases from a to e). The GDD stretches a pulse in time, such that the instantaneous frequency in the pulse changes linearly with time. At zero GDD (c) all the frequencies arrive at the same time, resulting in the shortest possible pulse.

In our simulations we have described the laser spectrum only in the $+\omega$ part and left it zero in the negative part, such that an interaction between a positive and negative $\omega$ can not take place. In this description we have implicitly taken the rotating wave approximation (RWA) ${ }^{27}$.

We have formulated our simulation such that only the SHG response is included in the signal and the OR is discarded. Thus, the simulated signal is the same signal that is measured experimentally.

\subsubsection{Chirp scans}

In a chirp scan the group-delay dispersion (GDD) on a pulse is scanned. GDD delays the blue side of the spectrum relative to the red side. The intermediate frequencies arrive at times between these extremes, such that the instantaneous frequency is a linear function of time (Figure 3.3). We will study the SHG response to a chirp scan and the influence of a resonance on the shape of a curve measured in this way. We find that this scan gives more insight into the resonance than an autocorrelation.

First, a practical advantage of a chirp scan over an autocorrelation is the smooth second-order phase profile of a chirp. This profile can be applied to a pulse with a pixelated pulse shaper without significant distortion. To synthesize the pulse pair for an autocorrelation with a pulse shaper a phase and amplitude mask with sharp features is required. A pulse shaper does not produce these features with a high accuracy. Performing an accurate autocorrelation calls for additional equipment, i.e. an interferometer, to be included in the setup that is not necessary for a chirp scan.

Using the same approach as for the autocorrelation, we simulated the SHG signal for a range of chirps on the pulse. We compare the 


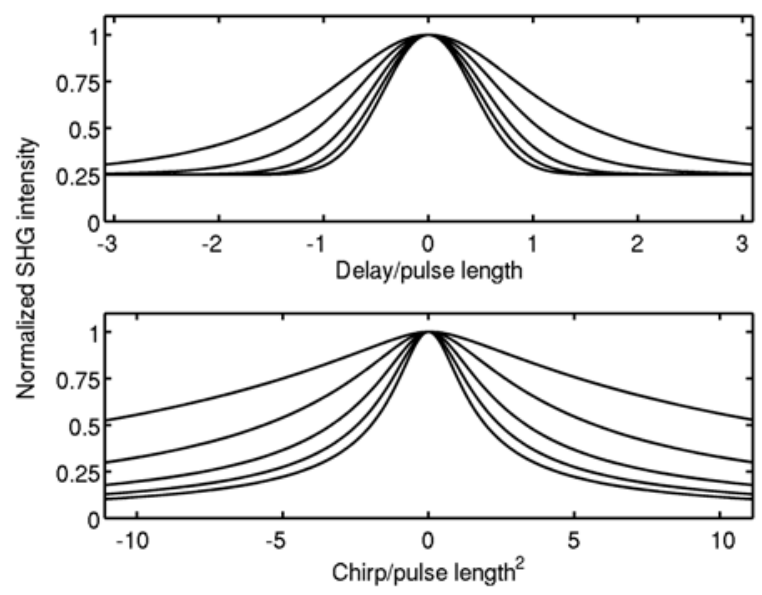

Figure 3.4: Scans of autocorrelation (top) and chirp (bottom) on resonances with lifetimes of 2, 1, 0.5, 0.25 and 0 (non-resonant) times the pulse length. Longer lifetimes correspond to broader peaks. The chirp scans start from a slightly narrower peak for the non-resonant case, but broaden much more compared to the autocorrelation scans as the lifetime is increased.

traces to the envelope, i.e. with the interference removed, of autocorrelation traces on the same resonance.

The results are shown in Figure 3.4, with the autocorrelations on top and the chirp scans in the bottom Figure. The non-resonant case corresponds to the narrowest peak in both Figures. As the lifetime of the resonance is increased, all peaks broaden. The curves corresponding to the chirp scan and autocorrelation are of similar width in the non-resonant case. However, as the resonance lifetime increases the chirp scan traces broaden more than the autocorrelation curves, which shows that the chirp scan is more sensitive to the presence of a resonance.

In the curves in Figure 3.4, the resonance is in the center of the laser spectrum. When the resonance is not centered in the spectrum, the shape of the chirp scans changes. This change is due to the phase profile associated with the resonance. Figure 3.5 shows the amplitude and phase for a resonant lineshape. The phase runs from 0 (following the driving force) for excitation frequencies below the resonant frequency to $\pi$ (lagging half a cycle behind the driving force) for frequencies above the resonance. When the laser spectrum is shifted to a lower or higher frequency from the center of the resonance, the excita- 


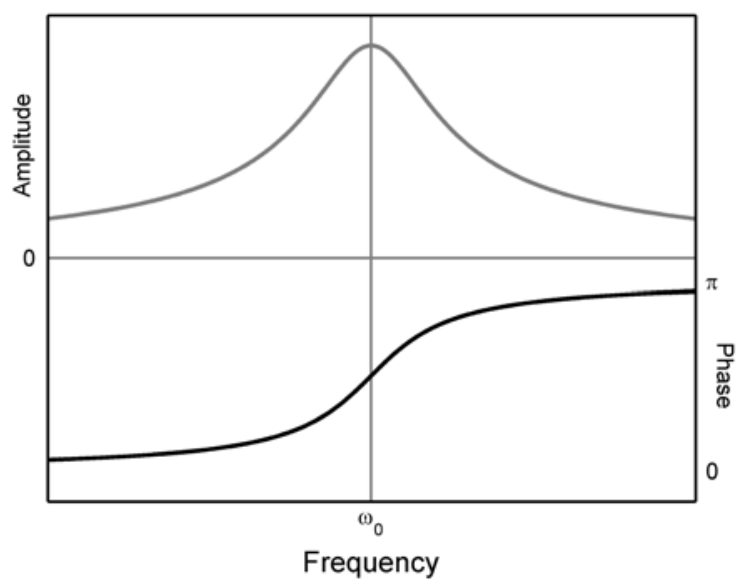

Figure 3.5: A resonant lineshape and its associated phase. The chirp scan is sensitive to the curvature in the phase, which can be exploited to determine the position of the resonance relative to the spectrum of the excitation pulse.

tion field experiences more of the curvature in the phase on one side than on the other side. The maximum of an autocorrelation trace is not affected by a detuned excitation. The trace will only narrow slightly, because the overlap between the resonance and the laser spectrum is reduced also the effect of the resonance on the width of the trace is reduced.

The curvature in the phase response of the resonance is similar to the phase profile used in the chirp scan itself. There is a point in the scan where this curvature is compensated by the chirp introduced in the scan. The chirp scan will produce a maximum in the SHG at this point instead of at zero GDD. For which GDD exactly the chirp scan compensates the phase curvature depends on the width of the resonance and how far off-resonant the excitation is. Figure 3.6 shows the shift of the maximum for a number of widths for the resonance and a range of detunings. For slightly off-resonant excitation, there is a large shift of the maximum in the chirp-scan peak. In this situation, the transform-limited pulse does not give the maximum SHG response. This effect was first seen in the two-photon excitation of Rubidium ${ }^{28}$.

In addition its sensitivity to the lifetime of the resonance, the chirp scan technique also gives information on the position of the resonance relative to laser spectrum. 


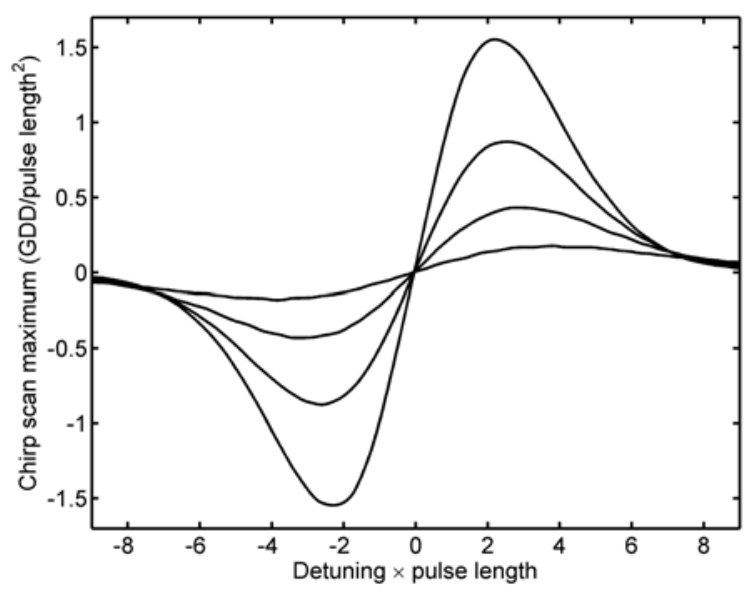

Figure 3.6: The shift off the maximum of a chirp scan as a function of detuning. The resonances have lifetimes of 2 (maximum shift), 1, 0.5 and 0.25 times the pulse length.

\subsubsection{Homogeneous and Inhomogeneous line widths}

Distinguishing the homogeneous and inhomogeneous broadening of a resonant line width is the goal of many spectroscopic techniques ${ }^{8}$. The homogeneous line width is the width of a single oscillator in the sample, which is inversely related to the lifetime of the oscillation. The inhomogeneous broadening is the amount a resonant line is increased due to the presence of oscillators with differing frequencies resulting from inhomogeneities in the sample. With a measurement that can separate these effects we can learn about the properties of the individual oscillators and how they are affected by their local environment.

To measure the purely homogeneous line width a non-linear technique is required, in which the inhomogeneous broadening is cancelled out. A non-linear technique in which a difference frequency is generated, i.e. mathematically a frequency at $-\omega$ interacts with a frequency component at $\omega$, can be used to cancel this broadening ${ }^{8}$. In Section 3.3.1 we mentioned that in the full second-order field there is such a term. Including this term in the simulation of SHG led people to believe that SHG could be used to determine the homogeneous line width. Unfortunately, this term can not be measured with the same detector as the SHG light and therefore the information it provides is lost. 


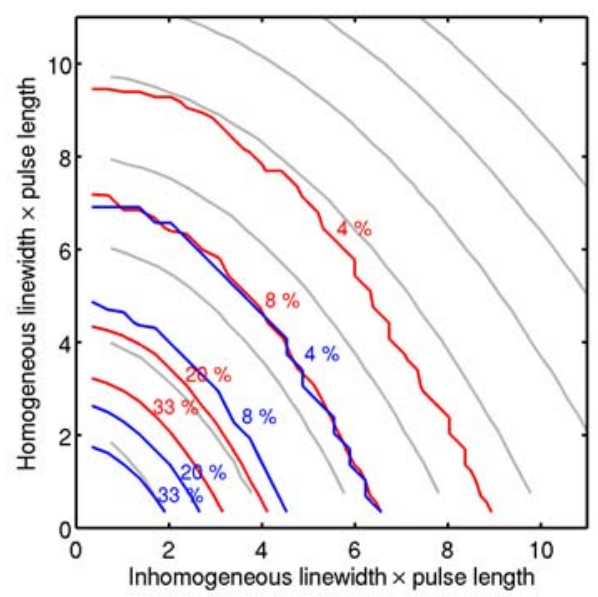

Figure 3.7: Comparison of the peak broadening of an interferometric autocorrelation (blue) and a chirp scan (red). For each technique the contour lines that give rise to a broadening of 4\%, $8 \%, 20 \%$ and $33 \%$ are indicated. The gray lines show combinations of homogeneous and inhomogeneous broadening resulting in the same FWHM in the linear spectrum. These lines are plotted on the the scale of a $21 \mathrm{fs}$ long input pulse in comparison with the non-linear measurements.

In the following we will do the simulation in accordance to what is measured in the experiment and show that it is not possible to distinguish the two contributions to the width of the resonant line. Both the autocorrelation and the chirp scan methods will be tried. We will make a contour map of the width of the peak calculated for both techniques for different combinations of homogeneous and heterogeneous broadening, similar to the work of Vartanyan et al. ${ }^{29}$.

We modify the spectral response of the resonance in equation 3.9 by including a distribution of center frequencies:

$$
x_{0}(\omega)=\frac{1}{\Delta \sqrt{\pi}} \int_{-\infty}^{+\infty} \frac{e / m}{\Omega^{2}-\omega^{2}+2 i \omega \Gamma} \exp -\frac{\left(\Omega-\Omega_{0}\right)^{2}}{\Delta^{2}} d \Omega,
$$

in which $\Gamma$ is the homogeneous line width, $\Delta$ is inhomogeneous width and $\Omega_{0}$ is the center frequency of the distribution. We calculated the autocorrelation and chirp scan traces for a range of combinations of $\Gamma$ and $\Delta$. In Figure 3.7 a contour plot of the width of these traces is shown. The axis is this plot are the dimensionless parameters, lifetime $\times$ pulse 
length. Any point on a contour line will give the same width in the SHG scan. It is not possible to determine which combination of $\Gamma$ and $\Delta$ led to this width. There is no difference between the shapes of the contours for the autocorrelation and the chirp scan. The only difference is that, because the chirp scan is more sensitive to the resonance, a larger increase in the width of the trace compared to the non-resonant width is observed for the same resonant line shape.

It was also suggested that a combination of a second-order measurement and a linear measurement could determine the homogeneous and inhomogeneous line width ${ }^{25}$. To check this suggestion, we have included the line width that would be measured by a linear technique in gray. The non-linear data are plotted for dimensionless parameters that depend on the pulse length, while the linear data do not depend on the pulse length. Therefore we have included the linear data on a scale that corresponds to an experimentally reasonable pulse length of $21 \mathrm{fs}$. The shapes of the linear and non-linear curves are nearly identical. Combining two measurements to determine the combination of $\Gamma$ and $\Delta$ requires one to find the intersection between a contour line of a linear measurement and a non-linear measurement, i.e. two nearly parallel lines. Two nearly parallel lines with some uncertainty on their positions will result in a large error on the position of their intersection. Thus, the combined measurement will not give an accurate measure of the line widths underlying the resonance. 


\subsection{Shaping the SHG fitness landscape}

In the previous sections we have only worked with a linear chirp on the pulses. For the optimization of some physical systems the linear chirp may be too limited. Non-linear chirps, in which the phase as a function of frequency scales with a cubic or higher power, can of course also be applied in phase shaping. In fact, according to the Taylor theorem, any analytic spectral phase function can be expressed via a series of constants, times the frequency raised to different powers.

$\phi(\omega)=\phi\left(\omega_{0}\right)+\frac{\phi^{(1)}\left(\omega_{0}\right)}{1 !}\left(\omega-\omega_{0}\right)+\frac{\phi^{(2)}\left(\omega_{0}\right)}{2 !}\left(\omega-\omega_{0}\right)^{2}+\frac{\phi^{(3)}\left(\omega_{0}\right)}{3 !}\left(\omega-\omega_{0}\right)^{3}+\cdots$

In this equation, the linear chirp is the $\phi^{(2)}(\omega)$ term. The two lower order terms have no influence on the pulse shape for a pulse that is relatively long compared to the center wavelength. The $\phi^{(1)}(\omega)$ term changes the arrival time of the pulse and the phase offset, $\phi\left(\omega_{0}\right)$, determines the offset between the field oscillations and the envelope of the pulse. These two terms do not influence the intensity of SHG, as was derived in Section 3.2.

In the optimization of a phase shaping experiment, the Taylor coefficients are often used as the parameters that are tuned to reach the optimum. In this section we will study the landscape of the SHG optimization problem when this basis set is used. Furthermore, we will present a modified basis set that results in a fitness landscape with improved properties from the point of view of an optimization.

The fitness landscape is the intensity of non-resonant SHG as a function of the parameters used in an optimization. When the Taylor series is used as the parametrization, the Taylor coefficients are the parameters. We will denote these parameters by $\phi_{2}, \phi_{3}, \phi_{4}$. A fitness landscape has as many dimensions as the number of parameters. We will try to gain insight into the landscape by looking at 2-dimensional projections of just one parameter versus another, while all other parameters are zero.

Figure 3.8 shows the 2-dimensional fitness landscapes for combinations of parameters for phase functions with opposite parity (even versus odd). The range ( -1 to 1 ) of the parameter scan is chosen to correspond to the points in the scan were the SHG intensity is half that of the maximum. The landscapes in Figure 3.8 are all mirror-symmetric 


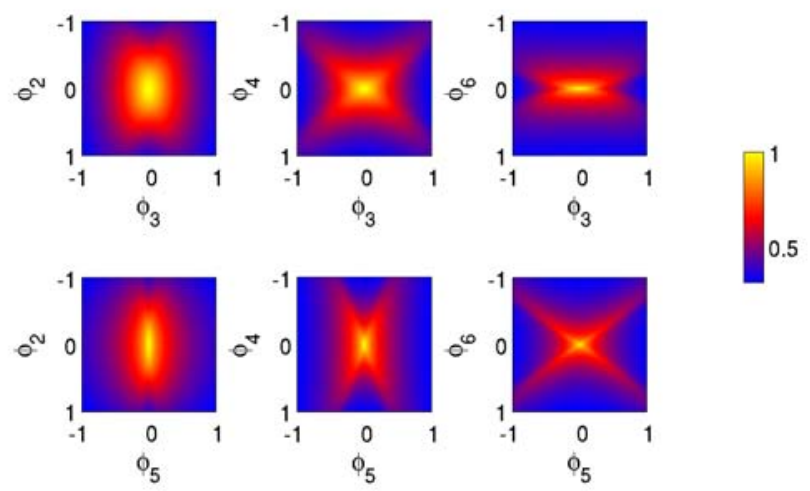

Figure 3.8: 2-dimensional maps of the SHG intensity for combinations of polynomial phase orders with opposite parity. The maps are symmetric around the optimum, such that this optimum can be found by one dimensional scans of the parameters.

around the optimum. Exploiting this symmetry, the optimum can be found by just two independent single parameter scans. For each scan either a single, or two maxima are found. In the case of a single maximum, the value giving rise to this maximum also yields the global maximum. For the case of two maxima, the average of the two values giving these maxima will yield the global maximum. However, a search algorithm may not be aware of the the maxima and could be attracted to just one of them, lengthening the search path. For such an algorithm, a landscape with convex contour lines would give a better result.

Figure 3.9 shows similar fitness landscapes as Figure 3.8, but now the two parameters are for phase functions with the same parity (both even or both odd). In this case, there is no symmetry around the maximum and the maximum can't be found via independent scans of the parameters. Parameters with the same parity can compensate for each other such that a positive value for one combined with a negative value for another yields an SHG intensity close to the maximum. This property of the fitness landscape may give an optimization algorithm difficulty to converge on the optimum, as an error in one parameter can be masked by an other parameter. Furthermore, close inspection of the landscapes reveals that there is a considerable non-linear shear in the landscape. A convex projection with principle axis parallel to the parameter is shear as if two oppositely directed forces are pulling on the 

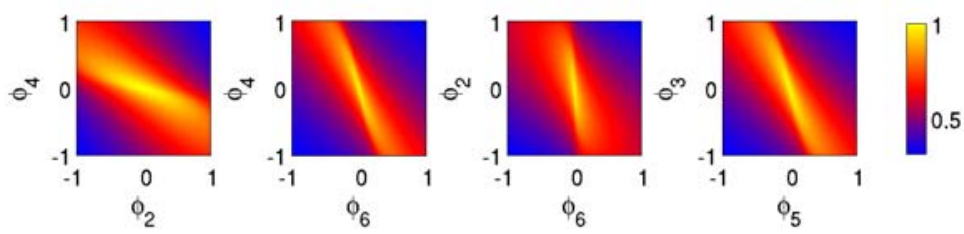

Figure 3.9: 2-dimensional maps of the SHG intensity for combinations of polynomial phase orders with the same parity. The maps are asymmetric around the optimum, such that this optimum cannot be found by one dimensional scans of the parameters. An error in one parameter is compensated by an error in another parameter.

image along opposite sides. In a non-linear shear the displacement of the image is a non-linear function from one side of the image to the other A landscape with non-linear shear will lengthen the search path followed by an algorithm and increase the computation or measurement time. For example a steepest decent algorithm has to follow a curved path on such a landscape. For each linear section along this path the algorithm has to determine the local gradient, a computationally costly procedure.

\subsubsection{Constructing a basis set}

We will choose a landscape with a more convex shape to find the optimum more easily, either via one dimensional scans, or via the use of a search algorithm. We will start by choosing a better $2^{\text {nd }}$ order versus $4^{\text {th }}$ order landscape and then look at the consequences for the other landscapes. First, we correct the non-linear shear via the introduction of a linear shear. Then we will rotate the basis to have the principle axis of the landscape parallel to the basis functions. Figures 3.10a-e show the effect of changing the $4^{\text {th }}$ order basis function by subtracting increasing amounts of the $2^{\text {nd }}$ order basis function. At figure 3.10e the amount of twisted contours in the landscape is greatly reduced and a landscape with minimum non-linear shear is found. The shape of the maximum is still at an angle with the basis functions. We correct this tilt by another linear shear; we change the $2^{\text {nd }}$ order basis function by subtracting the $4^{\text {th }}$ order function. The result of this rotation is shown in Figure 3.10f. Finally we rescale the new $4^{\text {th }}$ order basis function by a 

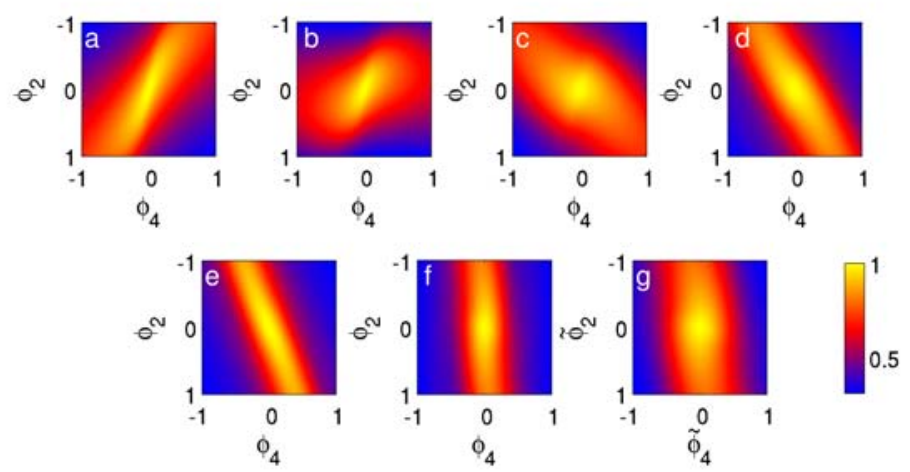

Figure 3.10: A new $2^{\text {nd }}$ order versus $4^{\text {th }}$ order landscape is created by using a combination of the two basis functions. First, an increasing amount of $2^{\text {nd }}$ order is subtracted from the $4^{\text {th }}$ order (a-e), until a landscape with minimal non-linear shear is obtained for $\omega^{4}-0.56 \omega^{2}$. This landscape is then rotated by subtracting the $4^{\text {th }}$ order from the $2^{\text {nd }}$ order $\left(\omega^{2}-4.96 \omega^{4}\right.$, Figure 3.10f). Finally, the $4^{\text {th }}$ order basis function is scaled by a factor of $0.6(\mathrm{~g})$.

factor of 0.6 to have a similar steepness along both axis.

By creating the new $2^{\text {nd }}$ order versus $4^{\text {th }}$ order landscape, we have changed the shapes of other 2-dimensional landscapes that include these two functions. In Figure 3.11 we compare the original landscapes (top) with the result of the new basis set (bottom) for these landscapes. The result of the modified basis also turns out favorably for the $4^{\text {th }}$ order versus $3^{\text {rd }}$ order landscape, because a shape with convex contour lines is produced. The $2^{\text {nd }}$ order versus $3^{\text {rd }}$ order landscape has qualitatively not changed as a result of the correction. Overall the properties of the landscape have improved in convexity, orthogonality and a reduced non-linear shear. The procedure can be repeated to include higher order terms if required. 


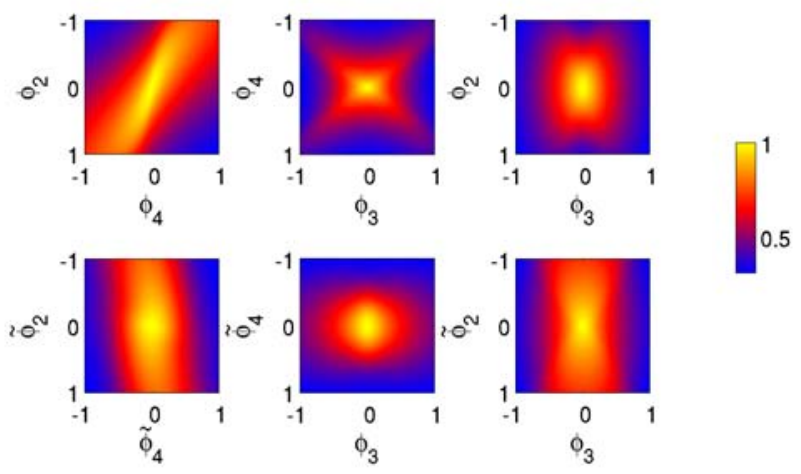

Figure 3.11: The original landscapes (top) and the landscapes as a result of the correction of the $2^{\text {nd }}$ order versus $4^{\text {th }}$ order landscape (bottom). In addition to the landscape that was worked on, also the $4^{\text {th }}$ order versus $3^{\text {rd }}$ order landscape was improved, while the $2^{\text {nd }}$ order versus $3^{\text {rd }}$ order landscape has similar properties as the original landscape.

\subsubsection{Conclusion}

A pseudo-orthogonal basis set has been determined for the optimization of the SHG. Even though the underlying process is non-linear and has no usable linear operator approximation ${ }^{19}$, a basis set exists that produces a landscape with convex contour lines and independent parameters around the optimum. Using this basis set in an optimization will result in a faster and more reliable convergence on the global optimum. 


\section{Chapter 4 \\ SiMULATION OF LIGHT ON Planar Metal NANOSTRUCTURES}

In this chapter, a simulation method for planar metal nanostructures is introduced. We test this method and apply it to random metal nanostructures. The fill ratio of the metal is varied and it is found that at the percolation threshold the structure generates the best response to have selective local excitation. Finally, the chirp scan method developed in the previous chapter is applied to a percolation structure. 


\subsection{Planar metal nanostructures}

Metal nanostructures strongly interact with optical radiation ${ }^{30}$. We will study this interaction on ultrafast time-scales ${ }^{31}$ and its dependence on the shape of the metal structure. The possible shapes of a structure are infinite. For practical applications two constraints have to be taken into account. First, it has to be possible to create the structure and second, one has to be able to perform an experiment on the structure. Mainly for the first reason pseudo-two-dimensional structures are popular. Borrowing techniques from the semiconductor industry, structures in a plane can be easily created with a high resolution. In our experiments we also use structure fabricated via these techniques (electron-beam or focused ion-beam lithography). To simulate the electric field on these planar metal nanostructures, we consider them as two-dimensional electrical networks ${ }^{32-34}$. The simulation method is described in detail in the following section.

\subsection{Simulation method}

We have simulated the interaction of light with metal nanostructures in the quasi-static limit. In this limit the retardation effects of optical waves are neglected, assuming the structure is smaller than a wavelength ${ }^{35}$. We have modeled the structure as an electrical network on a square lattice. The metal was mimicked by elements with complex conductivity derived from a Drude fit to the response of the bulk metal. As these lossy elements have mostly resistive properties, they are represented by resistors in Figure 4.1. The gaps in the metal structure were modeled with capacitors with complex conductivity derived from the permittivity of the substrate. At each lattice site in the network the current is conserved (Kirchhoff's current law). Imposing this condition on each site leads to the following equation:

$$
\sum_{j} \sigma_{i j}\left(\phi_{i}-\phi_{j}+E_{i j}\right)=0
$$

Where $\sigma_{i j}$ represents the conductivity between nodes $i$ and $j, \phi_{i}$ is the electrostatic potential at node $i$ and $E_{i j}$ is the externally applied field across the connection between $i$ and $j$. The equation can be rearranged to a matrix equation by taking the external field to the other side of the 


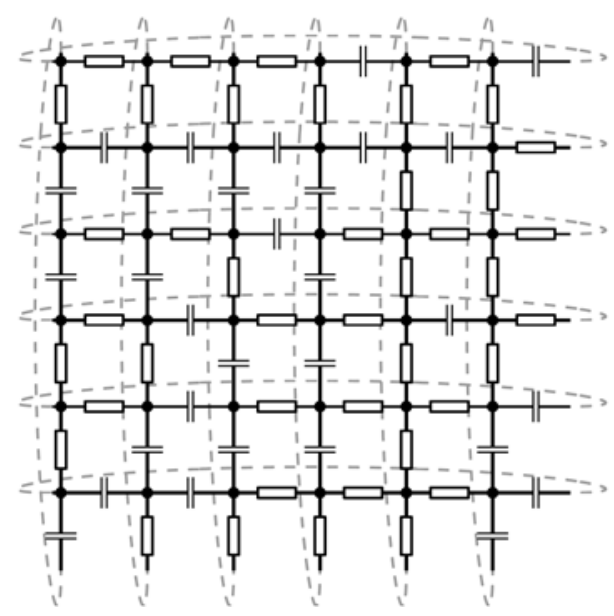

Figure 4.1: Electrical network mimicking a planar metal structure. Periodic boundary conditions where used in the simulations.

equals sign.

$$
H_{i j} V_{j}=F_{i}
$$

Here $H_{i j}$ is an $N^{2} * N^{2}$ matrix, for a structure size of $N^{2}$ nodes, and $V_{j}$ are the electric potentials at each lattice site. The matrix equation can in principle be solved via a Gaussian elimination method. However, we have solved the equation in a slightly more efficient way by taking into account the structure of $H^{36}$. This matrix is very sparse, because sites are only connected to their neighbours and thus conductivity to all other sites is zero. Only some blocks of $H$ are non-zero (dashed squares in Figure 4.2). The elimination process only needs to work on these blocks, effectively reducing the matrix size from $N^{2} * N^{2}$ to $3 N *$ $N^{2}$.

To simulate excitation by a short pulse, we have to consider the range of frequencies that constitute the pulse. We simulated the response to such pulses by adding up the individual responses of the nanostructure to all frequencies contained in the pulse. To calculate the response at a single frequency, the matrix $H$ is constructed with the conductivity of the metal at this specific frequency and the system of equations is subsequently solved. The effect of pulse shaping can be efficiently studied using this approach. The settings of the pulse 


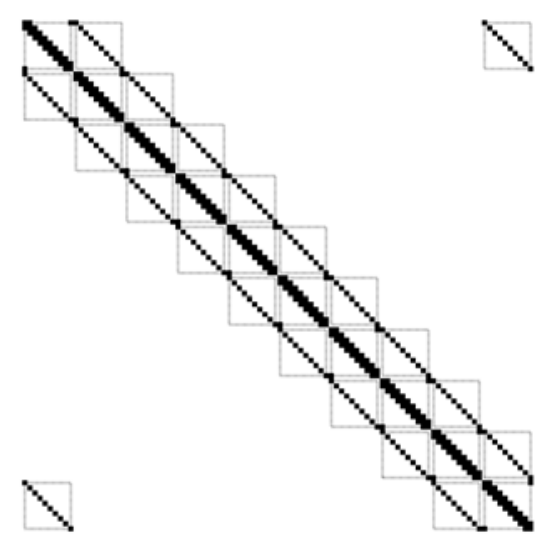

Figure 4.2: Map of the non-zero elements in the Kirchhoff matrix for a 10 by 10 grid. The Block Elimination method works only on the marked squares.

shaper (phase and amplitude) are simply multiplied with the (complex) responses at each frequency before adding them up. We will use this approach to calculate the local response on metal nanostructures when illuminated by phase-shaped pulses.

\subsection{Small metal particles}

Before continuing with complex nanostructures, we first test our simulation method on a case that has an analytic solution: a subwavelength particle. For particles much smaller than the wavelength of light, the quasi-static limit applies, i.e. the field can be considered constant across the particle. In this limit the polarizability of a metal sphere has an analytic solution that can be found via Mie theory ${ }^{37}$.

$$
\alpha=4 \pi \varepsilon_{0} V \frac{\varepsilon_{m}-\varepsilon_{d}}{2 \varepsilon_{d}+\varepsilon_{m}}
$$

In this equation, $\alpha$ is the polarizability, $V$ is the volume of the particle and $\varepsilon_{m}$ and $\varepsilon_{d}$ are the dielectric constants of the metal and the surrounding dielectric.

In the optical regime, the dielectric constant of a metal depends strongly on the frequency of the light. Figure 4.3 shows the real and 


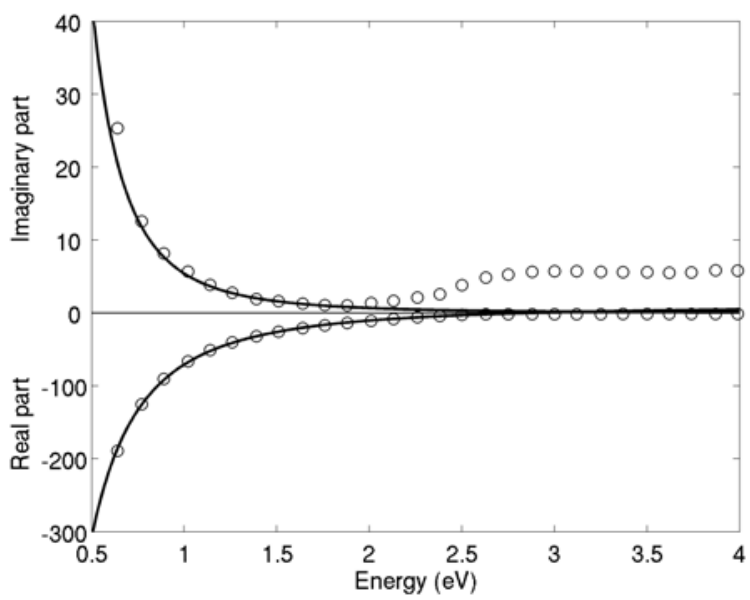

Figure 4.3: Real and imaginary part of the dielectric constant of bulk gold (circles) and a fit to the data points according to the Drude model (solid lines). The deviation for energies above $2 \mathrm{eV}$ is due to the excitation of an inter-band transition, causing an increased damping.

imaginary part of the measured dielectric constant of bulk gold ${ }^{38}$. The solid lines are a fit to the data using the Drude model ${ }^{39}$. This model works well for energies below $2 \mathrm{eV}$, but breaks down at higher energies due to the excitation of the tail of an interband transition. Energy is lost due to this excitation, causing an increase in the damping rate. We have limited our experiments to the range where the Drude model works well and therefore use this model in the simulations.

To model a small spherical particle in a surrounding dielectric, we take the grid-points within a sphere on a square lattice and interconnect these points via resistors. The other lattice points are connected to their neighbours via a capacitor. We calculated the total field intensity on this structure for a range of incident frequencies. The result has a large peak around $520 \mathrm{~nm}$ with some smaller peaks at shorter wavelengths (Figure 4.4). We can compare this result with the analytical expression given by equation 4.3. Using this expression, we obtain a single peak that coincides with the large peak from the simulation (black line in Figure 4.4). The deviation at shorter wavelength is due to the shape of the particle in the simulation. On a square grid the sphere is only approximated and higher order modes exist on this particle. 


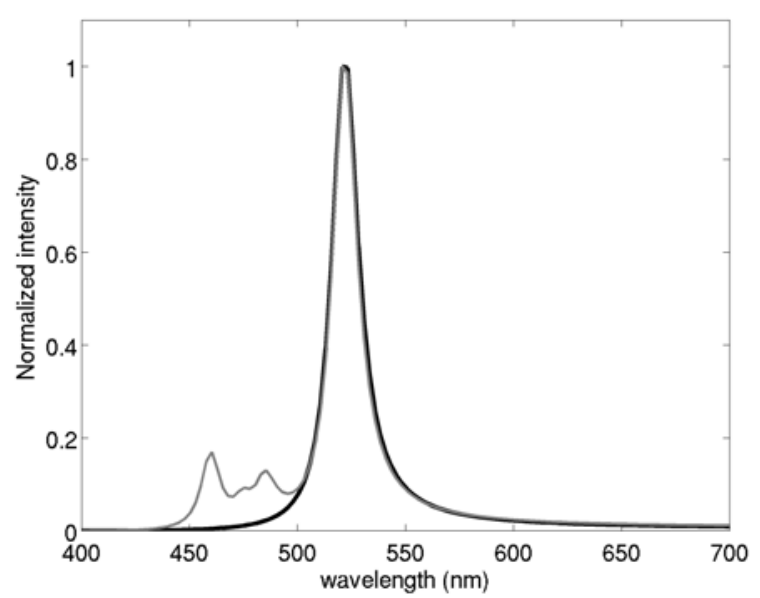

Figure 4.4: The field enhancement for a small spherical gold particle using the analytical expression (black) and the simulation (grey). A good agreement is found for the main resonance. The deviation at shorter wavelength is due to the shape of the particle in the simulation. The spherical shape is approximated on a square grid.

\subsection{Percolation structures}

Random metal-dielectric composites are an area of active study ${ }^{40}$. The rough structures caused by the random positioning of materials cause strong field enhancements. These strong fields make the structures useful for non-linear optics and non-linear spectroscopies. The percolation threshold seems to play an important role in the distribution of the field intensities on a structure ${ }^{41}$.

Starting from a dielectric substrate and adding small amounts of a metal, the metal first forms disconnected islands. As the amount of metal is increased, at some point the individual islands start to connect. The amount of metal required to just reach a state were all the metal islands are connected is called the percolation threshold. In this section we study the properties of a percolation structure when illuminated with phase shaped pulses.

\subsubsection{Spatial phase correlation}

We would like to selectively excite specific positions on the structure using shaped pulses. For this purpose we can apply the principle of 

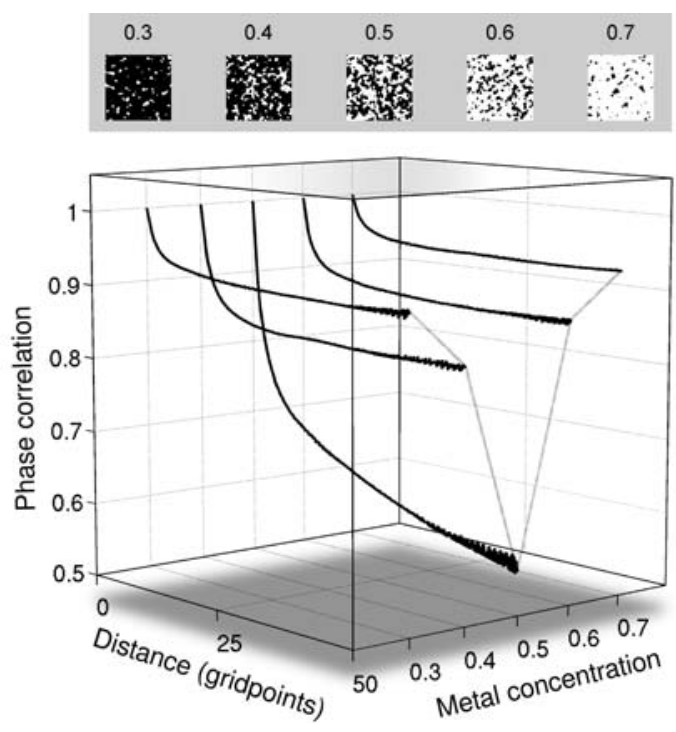

Figure 4.5: The spatial phase correlation function (bottom) for five structures with increasing gold concentration (top). The correlation function drops the most for a structure with a gold concentration of 0.5, i.e. at the percolation threshold.

time-reversal ${ }^{42,43}$. The position we would like to excite has a certain impulse response. If we excite the structure with the time-reversed impulse response, the selected position will experience a short intense field that is transform-limited. A non-linear process that is a small perturbation to the otherwise linear system, such as two-photon absorption, is sensitive to the peak intensity of the field and will be maximal when the local field is transform-limited.

To selectively excite different positions, they need to have a different impulse response. We tested how the local response correlates on a few random structures with different gold concentrations (Figure 4.5). Experimentally, we can not create the true time-reversed response, as we are limited by the input laser spectrum. Under this constraint, a non-linear process is maximized when just the phase of the response is inverted and the amplitude is kept constant.

We will determine how selective the excitation is, when we pick a position $r_{0}$ and illuminate the sample with the spectral phase $-\phi\left(\omega, r=r_{0}\right)$, i.e. the inverted spectral phase of the local response at $r_{0}$. We calculate the efficiency of the excitation of a second-order non-linear process at each position, relative to the maximum possible 


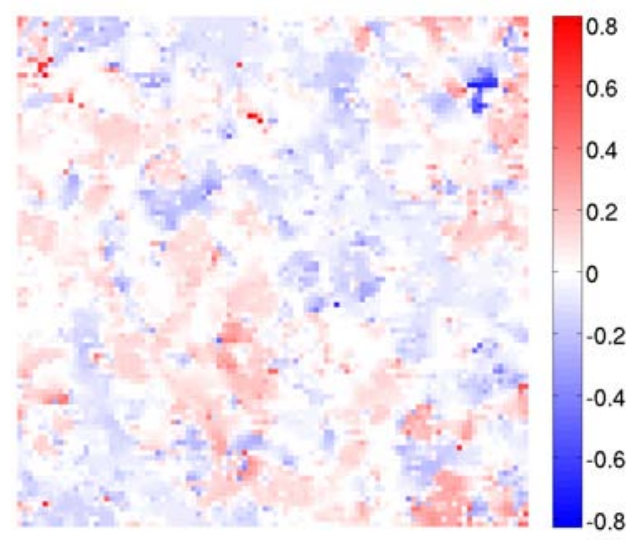

Figure 4.6: The shift of the chirp scan peak in units of the non-resonant width of the peak. Large shifts are visible, both to the left and right.

excitation of this process at the same position.

$$
C\left(\left|r-r_{0}\right|\right)=\left\langle\frac{I^{(2)}\left(r,-\phi\left(\omega, r=r_{0}\right)\right)}{I^{(2)}(r,-\phi(\omega, r=r))}\right\rangle .
$$

In this equation $I^{(2)}(r, \phi(\omega, r=r))$ means the intensity of the secondorder non-linear response at position $r$ when the structure is excited with the spectral phase $\phi(\omega, r=r)$, which is the local phase response at position $r$. The average is over all positions, $r_{0}$. The phase correlation curves for five different structures are shown in Figure 4.5.

In our simulations, we changed the concentration of gold on a dielectric surface from 0.3 to 0.7 . At a concentration below 0.5 , the structure consists of disconnected islands of gold, while at a concentration higher than 0.5 it contains disconnected holes in a gold layer. Exactly at a concentration of 0.5 , the limit is reached that both materials (gold and dielectric) can be connected. We see this behaviour mirrored in the correlation curves in Figure 4.5. The correlation curve for a concentration of 0.5 decreases the most, while the other curves level off at some value. This levelling off is due to the local responses in the disconnected islands (or holes) being similar.

At the percolation threshold, the local response has the most variation. Thus, a percolation structure is ideal for the selective excitation 


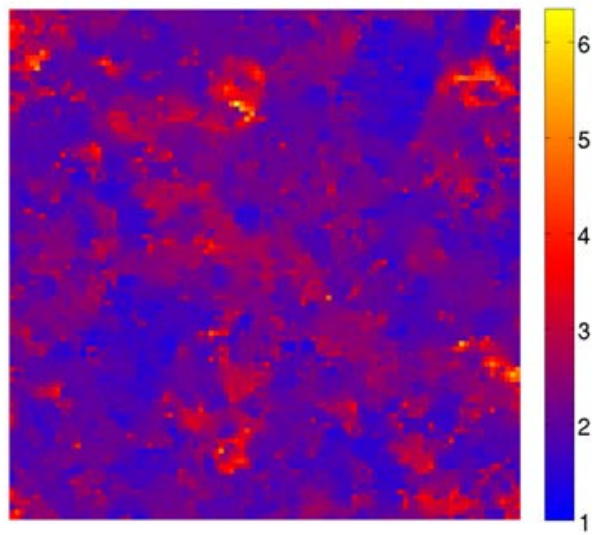

Figure 4.7: The width of the chirp scan peak relative to the non-resonant width. Very narrow resonances are present on the structure, which broaden the chirp scan peak by up to 6 times.

of different positions on a surface.

\subsection{2 chirp scan on a percolation structure}

In the previous section we found that the best structure to be controlled via the spectral phase is a percolation structure. We continue to study the effect of simple shaped pulses on the structure. The percolation structure from the previous section is used (Figure 4.5 top-middle) and subjected it to a chirp scan. We calculate the second-order field intensity for a set of pulses with increasing chirp-rate (both negative and positive). The input spectrum is centered at $675 \mathrm{~nm}$ and has a transform-limited pulse length of 40 fs. The plot of field intensity versus chirp-rate (or group-delay dispersion) yields a curve with a peak near the center (see Chapter 3).

Resonances on the structure distort the shape of the chirp scan curve and move its maximum away from the center. Figure 4.6 shows the shifts of the maximum relative to the width of the peak. Figure 4.7 shows the width of the peak relative to the width that would be obtained on a non-resonant sample. This Figure shows widths up to six times wider than the non-resonant case, indicating the presence of narrow resonances on the surface. Figures 4.6 and 4.7 show that the local response on a percolation structure strongly distorts the input pulse 
and that this distortion fluctuates with the position on the surface. The local response to an external field varies across the surface, which can be exploited for selective local excitation by tailoring the temporal profile of the illuminating field. 


\section{Chapter 5 \\ Phase Control over local FIELD INTENSITIES}

Nanostructured gold layers show localized field enhancement ('hot spots') when illuminated with broadband light. By coating such a film with a fluorescent reporter layer, we are able to image the local field intensity distribution. We illuminated a percolating gold structure with chirped pulses and scanned the chirp. The local response of the structure influences where this chirp scan shows a maximum. Since the maximum depends on the local response, this technique can be used to selectively excite regions on the surface of the nanostructure. 


\subsection{Introduction}

Nanostructured metal films have been widely used in surface enhanced spectroscopies such as surface enhanced Raman scattering (SERS) ${ }^{44}$. The highly enhanced local fields on such films increase the Raman intensity by orders of magnitude, pushing the sensitivity into the single molecule regime ${ }^{45}$. The intense local fields are due to localized resonances ('hot spots') naturally occurring on the nanostructured metal film ${ }^{46}$. To gain more control over the enhancement, nano fabrication techniques have been used to prepare the surface ${ }^{47}$. Controlled fabrication methods have yielded a wide variety of surface structures, including self-organized nano-particle arrays ${ }^{48}$, lithographically produced arrays of shaped particles ${ }^{49}$, and particles on top of gold films ${ }^{50}$. To complement this approach of passive control by carefully engineering the structure, active control of the dynamics of plasmons was proposed ${ }^{51}$. By tailoring the properties of the illuminating light the dynamics of plasmons in a nanostructure can be influenced to localize in time. To control plasmon dynamics at ultrafast timescales, spectral shaping of the incident light has to be used ${ }^{31,52}$. By combining nanostructures and spectral shaping, full control over the electric field at the femto-nanoscale can be achieved ${ }^{53}$. Designing fields on this scale allows a nanoscale object (e.g. a nanocrystal or molecule) to be efficiently coupled to a propagating field, which is useful for a number of devices and the study of the dynamics of the nano-object itself ${ }^{54}$.

Here we investigate experimentally the possibility of control the local field on a surface using spectral phase shaping. We have designed a percolation type structure using elements on the scale of the diffraction limit (Figure 5.1). The sample consists of randomly positioned circular gold islands on a dielectric substrate. The average size of the islands is $200 \mathrm{~nm}$. This scale ensures that different responses of the structure are separated sufficiently to be distinguished by a conventional microscopy approach. While others have used a photo-electron emission microscope (PEEM) to detect the local field intensity on metal nanostructures ${ }^{55-58}$, this technique is only sensitive to the field on the metal and not on to the field on the dielectric gaps. We will use a reporter layer of quantum dots (CdSe/ZnS, Evident Technologies, ED-C11TOL-0540) that is deposited on the surface and measure the fluorescence from this layer. Assuming we obtain an even coverage of the surface by the quantum dots, we can detect the field intensity on the 


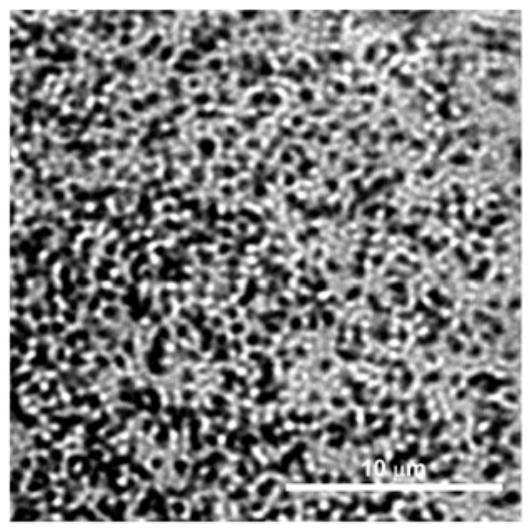

Figure 5.1: Sample of randomly placed gold islands imaged in transmission through the 100x 1.4 NA objective lens. The gold islands are visible in black.

glass in between the gold and on top of the gold. By using this approach, which relies on light microscopy, we suffer a loss in resolution compared to PEEM ( $\sim 40 \mathrm{~nm}$ for PEEM versus $\sim 250 \mathrm{~nm}$ for our optical detection method).

In a percolation structure, hot spots occur easily and over a wide spectral range ${ }^{59}$. On a random structure the local geometry (thus response) is different at each position of the sample. By using an excitation pulse that is tailored to a specific response, energy can be directed at the corresponding position on the sample. The principle of time reversal can be used to address a region on the surface ${ }^{42,43}$ (see also Chapter 4). By exciting the sample with the time-reversed response of this region, that location receives the maximum peak intensity.

We detect the local intensity by coating the structure with a reporter layer of quantum dots ${ }^{16}$ and image fluorescence from this layer. We have performed scans of the linear chirp on the pulse and measure the fluorescence intensity in response to these pulse shapes. We determine where in the scan a maximum is reached for each position on the sample. The results show that different positions on the sample can be addressed with different linearly chirped pulse. In contrast, the hotspots, positions with a high fluorescence intensity, all give a similar response to the chirped pulses and can not be individually addressed in this way. 


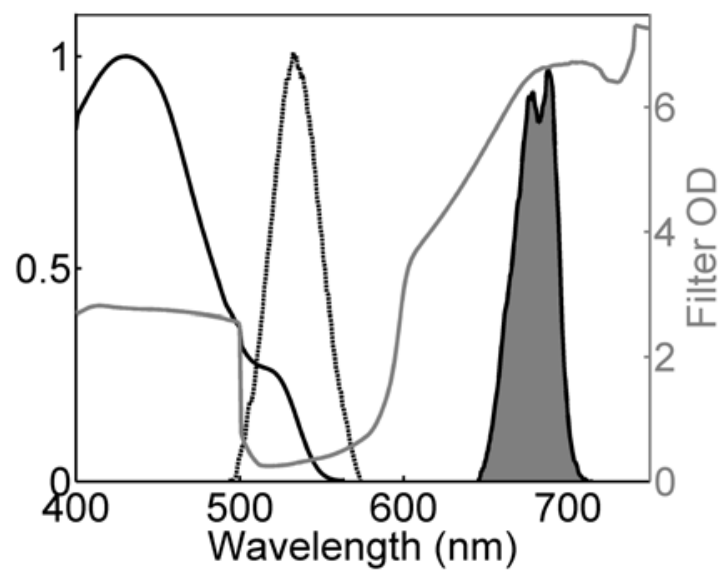

Figure 5.2: Absorption (full black) and emission (dashed) spectra of the quantum dots. The laser spectrum (shaded) and the optical density of the filters used on the microscope (gray). The laser has a longer wavelength than the absorbtion of the quantum dots, such that two photon are required to excite the dots. The optical filter block the laser light and only pass the fluorescence from the quantum dots.

\subsection{Experimental}

We fabricated a nanostructured gold film using electron beam lithography. The design of the structure was made by randomly positioning circular islands (diameter $200 \mathrm{~nm}$ ) within the write area. The density of the islands is such that an approximately $50 \%$ coverage by gold is achieved. After writing this design in a PMMA film, the film was developed and a gold layer of $40 \mathrm{~nm}$ thickness was deposited. A lift-off process dissolved the remaining PMMA producing the final gold structure.

The sample was dip-coated in a dilute solution of quantum dots (emission peak at $540 \mathrm{~nm}$ ) in toluene. The quantum dots form a reporter layer that visualizes local field intensities on the surface. A white light transmission image of the structure is shown in Figure 5.1. The experiments were performed with 35 fs laser pulses from a noncollinear optical parametric amplifier (NOPA) pumped by an amplified Ti:Sa system (Clark-MXR CPA 2001). The excitation pulses were centered at a wavelength of $685 \mathrm{~nm}$, far from the linear absorption of the quantum dots (Figure 5.2). Therefore, excitation of the quantum dots 
takes two photons and has a quadratic intensity dependence, making our technique sensitive to the peak-power of the response. The response of the quantum dots on a bare substrate to several pulse shapes was compared to the amount of second harmonic generated (SHG) in a BBO crystal. No deviations between the SHG light and fluorescence intensities were found, indicating that the two-photon absorption crosssection can be considered constant within the bandwidth of the pulses. The non-linear intensity dependence of the fluorescence is required to see any effect of phase-only shaping on the hot spot intensity. Because two-photon absorption scales with the peak intensity, the fluorescence signal of a hot spot is a measure of how the response is localized in time at that position.

The quantum dot coated structure was placed on a microscope and imaged on an EMCCD camera through a 1.4 NA oil immersion objective lens. A part of the structure was illuminated with the laser light by weakly focusing the laser on the structure. The fluorescence was detected through a set of filters that blocked all of the scattered laser light (Figure 5.2).

The laser pulses were passed through a 4-f pulse shaper with a programmable liquid crystal mask (CRI SLM-640). With this mask, an arbitrary spectral phase can be programmed on the excitation pulses. Before the experiments, the output pulses were compressed by iteratively finding the phase mask that maximized the amount of secondharmonic generated in a BBO crystal ${ }^{60}$. This phase mask was used as a background phase in the experiments. By applying a quadratic phase profile one of the simplest shaped pulses is produced, a chirped pulse. This pulse shape can easily be synthesized by sending a pulse through a dispersive medium. In the experiment, we scanned the chirp-rate from $-2000 \mathrm{fs}^{2}$ to $+2000 \mathrm{fs}^{2}$ in 64 steps. The pulse length in this scan went from 200 fs to the transform-limited 35 fs back to a 200 fs long pulse. For each pulse shape the image was integrated for 100 seconds on the camera. After each two pulses the transform-limited pulse was measured again to check if the quantum dots were bleaching. 


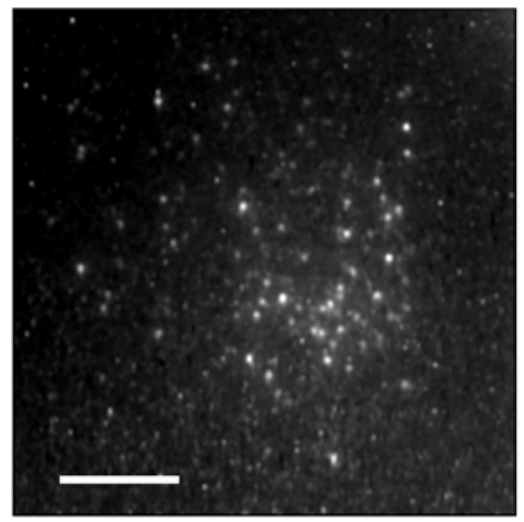

Figure 5.3: Two-photon excited fluorescence intensity from the percolation structure, showing the appearance of hotspots over a large area. The scale bar has a length of $10 \mu \mathrm{m}$.

\subsection{Results}

A typical image of the fluorescence intensity is

shown in Figure 5.3. It is clear from this image that localized hotspots are generated on this random structure. The hotspots are all very similar in intensity, which is surprising compared to what is predicted by theory ${ }^{34}$ and our simulations. The prediction is that the local intensity on a percolation type surface has a log-normal distribution. A spatial hole burning experiment on a SERS substrate showed a power law distribution of intensities ${ }^{61}$. The actual hotspots on the structure can be much smaller than the resolution they are detected at. For this reason their intensity is spread over a larger area, which may make their intensity to appear much more uniform.

It was not possible to reach a sufficient signal to noise ratio in a reasonable amount of time with the focus size used in Figure 5.3. Therefore, we used a tighter focus to do the chirp scan. Figure 5.4 shows the fluorescence when a smaller focus is used. Some hotspots are still visible. The chirp scan produces a similar image for each pulse shape. If we pick a position on the image and take the intensity from each of the stack of images measured with a different chirp, we obtain a chirp scan curve as was found in Chapter 3. The peak of this curve is somewhere around the transform-limited pulse. For which chirp the 
maximum intensity is reached exactly depends on the local response of the structure. Figure 5.5 shows the shift of the maximum away from the transform-limited pulse for the same area of the sample as in Figure 5.4. This Figure looks similar to Figure 4.6 in Chapter 4, however the amount of the shift is less than predicted in the simulation. Furthermore, if Figures 5.4 and 5.5 are carefully compared, it shows that there is no shift for any of the intense peaks. Finally, looking at the width of the peak produced by the scan, it does not deviate much from the width obtained with a constant (non-resonant) response. In the simulation (Figure 4.7) there is strong variation in the width of the peak, with widths up to six times that of a non-resonant peak.

\subsection{Discussion}

By applying a reporter layer we have clearly visualized the hotspots on a random gold nanostructure. We have investigated the influence of chirped pulses on the fluorescence intensity from the sample. We have seen that on some positions on the sample the peak of the chirp scan appears to the left or right of the transform-limited pulse. The shift makes it possible to individually address different positions on the surface by exciting with the pulse shape that gives the maximum at the desired position. The shift is, however, smaller than that predicted by our simulations. The reason for this discrepancy is that the simulations were performed in the quasi-static limit. In this limit the field is constant across the structure and does not couple to radiation modes in the far field. In the experiment, this radiative coupling is present and all resonances are significantly broadened by radiative damping.

The presence of radiative damping can be seen in the width of the chirp-scan peaks. The peaks are all close to the width that is obtained on a non-resonant sample, indicating broad resonances with a short lifetime. In addition, the points with the highest intensity did not show a shift in the peak at all, which may just be because these points have the best overlap with the laser spectrum. Another possibility may be that there is a correlation between the spectral and the spatial response of the sample in which the hotspots have a flat spectral response. However in the simulations such a correlation was not observed.

In Chapter 4 we concluded that the response of a percolation structure was optimal for the selective excitation of specific regions on the surface. The experiment shows, however, that the structure at the scale 


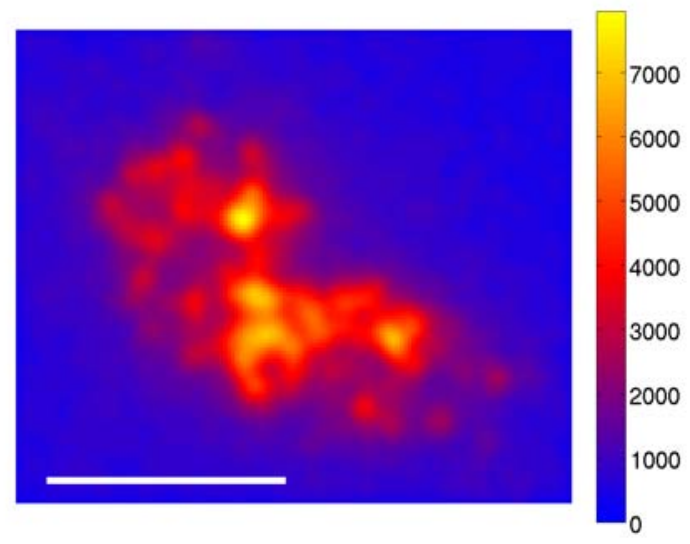

Figure 5.4: Fluorescence intensity of hotspots when a smaller focus is used. The scale bar has a length of $5 \mu \mathrm{m}$.

we produced it is not ideal. We have seen a weak possibility to excite some positions selectively, but the high intensity spots can not be used. To improve the structure, the radiative damping of the modes has to be controlled. The easiest route is to make the structure smaller, approaching the quasi-static limit. We have, however, reached the limit of our detection method using optical detection of the field intensity on the structure. The afore mentioned PEEM technique could be used to overcome this limit. Another route is to engineer the structure such that the modes interfere destructively in the far field by repeating the same structure with a spacing of one to a few wavelengths. In this way the modes can be controlled similar to the modes in a photonic crys$\operatorname{tal}^{62,63}$. 


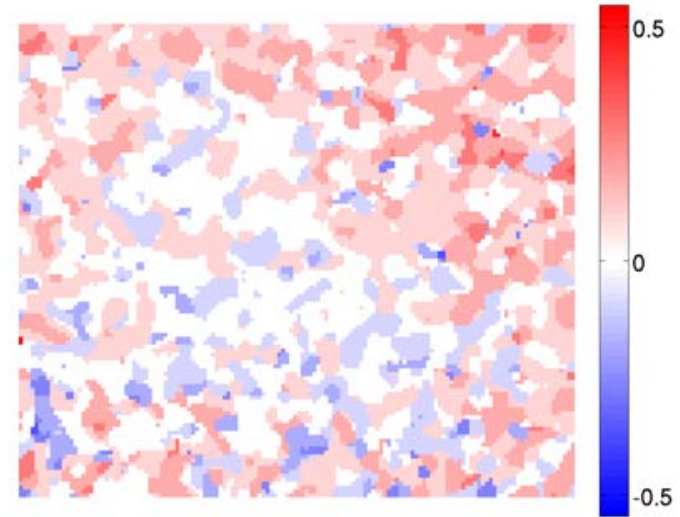

Figure 5.5: Shift from the center of the chirp scan peak. The image shows a similar pattern as the simulations in the previous Chapter (Figure 4.6) 


\section{Chapter 6 \\ SECOND-HARMONIC \\ GENERATION ON DISORDERED HOLE ARRAYS}

Metal films with periodic sub-wavelength perforations, socalled hole arrays, exhibit a much larger transmission than expected from the transmission of a single hole. Plasmonic resonances between the holes and inside the holes are the cause of this 'extraordinary transmission'. It was also found that hole arrays can produce SHG when illuminated with intense laser pulses. We investigate the mechanism behind the SHG by performing chirp scans. The analysis of the chirp scans shows a resonance with a long lifetime that is not visible in the linear transmission spectrum. 


\subsection{Introduction}

Metal films with periodic sub-wavelength perforations, so called 'hole arrays', are one of the most studied plasmonic structures. The interest in these structures was sparked by the discovery of the 'extraordinary transmission' (EOT) that such arrays exhibit ${ }^{64}$. For some incident wavelengths, the transmission through the array is larger than the open area of the holes. The transmission through a single hole scales roughly with $(r / \lambda)^{4}$ with $r$ radius of the hole and $\lambda$ the wavelength of the light ${ }^{65}$. Consequently, very little light will come through a single sub-wavelength hole, which makes the high transmissivity of the hole array an intriguing phenomenon.

The transmission spectrum of a hole array is highly structured; the transmission is enhanced for some wavelengths and suppressed for others. This spectrum also strongly depends on the incident angle of the light ${ }^{66}$. The position of the transmission peaks can be engineered by varying the pitch and hole shape of the arrays ${ }^{67,68}$. For these reasons sub-wavelength hole arrays have application potential as (directional) filters ${ }^{69}$.

The EOT is recognized to be caused by surface-plasmon polaritons (SPPs) that couple between the holes and by localized resonances inside the holes ${ }^{70,71}$. Resonance conditions exist for both the SPPs and for the light travelling through individual holes. These two contributions to the transmission interfere together, resulting in a highly structured transmission spectrum. Some resonances generate high fields on the structure, making non-linear effects possible. It was found that second-harmonic light was generated at hole arrays when illuminated with intense laser pulses ${ }^{72,73}$.

Van Nieuwstadt et al. ${ }^{74}$ investigated the role of the shape of the hole in the SHG process and found a 'magic' hole aspect ratio for which the SHG efficiency was increased by an order of magnitude. Prangsma et al. ${ }^{75}$ measured the group-delay for light propagation through the same hole shapes and found that the SHG intensity correlates with the transmission time of the light. In this Chapter we investigate the resonances underlying the SHG using the chirp scan method developed in Chapter 3. We study an ordered array and arrays with increasing disorder in the placement of the holes to understand the influence of the resonant coupling between the holes by SPPs. 


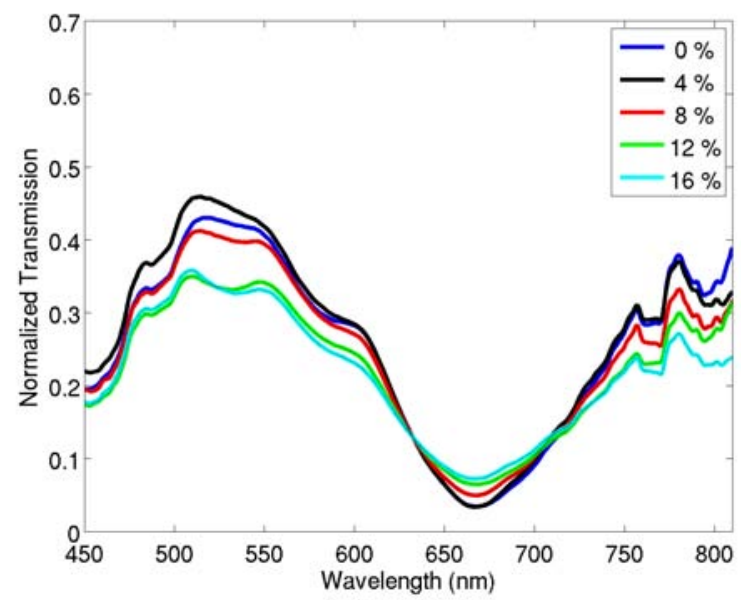

Figure 6.1: Normalized transmission spectra of the five hole arrays with increasing disorder. The transmission is normalized to the area of the open fraction of the array.

\subsection{Samples}

Five different hole array samples were produced by Focussed-IonBeam milling of square holes in a $200 \mathrm{~nm}$ thick gold film on a glass substrate. The hole size is $195 \mathrm{~nm}$ and the average pitch is $400 \mathrm{~nm}$ for all the arrays. The amount of disorder was varied in the arrays by adding a random offset with 0 to $16 \%$ root-mean-square deviation from the average pitch to each hole position.

The transmission spectra of the arrays are shown in Figure 6.1 and SEM images are presented in Figure 6.2. The light transmitted through an array was compared to the light transmitted through an open square of the same size as the array. Finally, the transmission was normalized to the open fraction of the hole array.

A dip associated with resonant plasmonic absorption, called the Woods anomaly ${ }^{76}$, is observed at $670 \mathrm{~nm}$. At this wavelength, the spacing of the holes matches the difference in the wavevector of the incident light and the wavevector of a surface plasmon. The general condition for this phase matching is:

$$
k_{S P P}(\omega)=k_{\|} \pm n \Gamma_{\text {lattice }},
$$




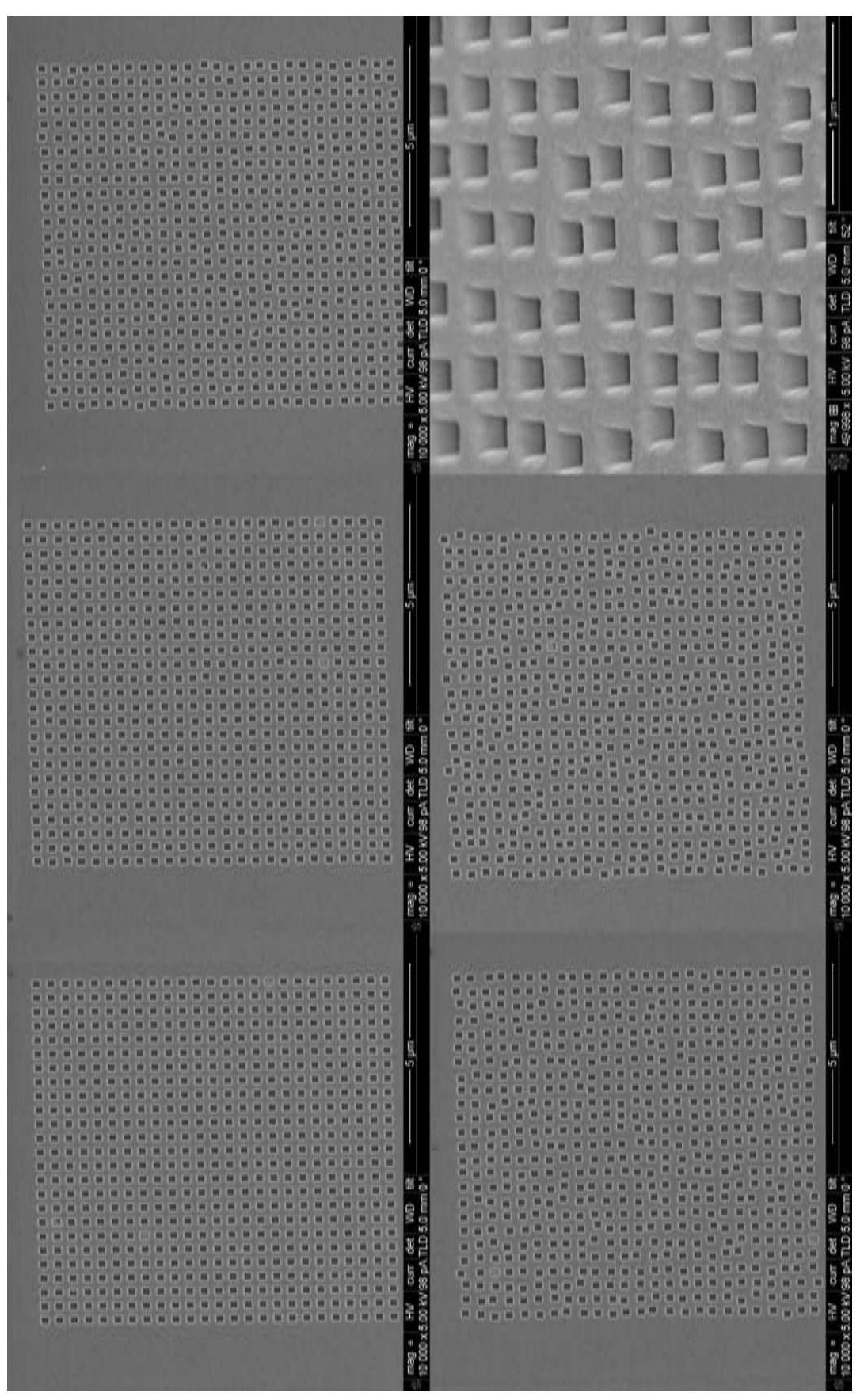

急焉

\&

응 웜

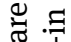

岁范

क⿺

号

들

들. 임

E. 죽

胥

잉

๘

$\cong \frac{\pi}{3}$

宊 
where $k_{S P P}$ is the wavevector of the surface plasmon, $k_{\|}$is the parallel component of the wavevector of the incident light and $\Gamma_{\text {lattice }}$ is a wavevector of the lattice.

The Woods anomaly is part of an asymmetric lineshape; a Fano lineshape ${ }^{77}$. This type of lineshape occurs when a resonance interferes with a continuum. Here, the resonant part is due to plasmonic coupling between the two sides of the film and the background continuum is direct transmission through the holes ${ }^{78,79}$.

The kink in at $600 \mathrm{~nm}$ in the spectra of Figure 6.1 is the Rayleigh anomaly. As in the measurement of these spectra, the light comes from the air side and exits on the glass side (refractive index 1.5), the first diffraction order propagates parallel to the sample surface (perpendicular to the propagation direction) for a wavelength of $600 \mathrm{~nm}$. Thus, if the wavelength is scanned from below $600 \mathrm{~nm}$ to above $600 \mathrm{~nm}$, the first-order diffraction disappears at $600 \mathrm{~nm}$. This change in the diffraction changes the slope of the transmission curve. The slower modulations around 500-550 $\mathrm{nm}$ and the sharp feature at $760 \mathrm{~nm}$ are caused by the highly structured spectrum of the light source (Xenon lamp).

As the disorder is increased, the total transmission decreases, while also the depth of the dip at the Woods anomaly decreases.

\subsection{Experimental}

The pulses from a Ti:Sapphire oscillator (Coherent Micra) were used in the SHG experiments. First the pulses were compressed by a combination of a prism-compressor and a pulse shaper. An evolutionary algorithm optimized the SHG from a BBO crystal to find the background phase profile to achieve a transform-limited pulse (see Chapter 3).

The pulses were focussed on the individual samples with a $0.03 \mathrm{nu}-$ merical aperture (NA) lens. At this NA the trade-off of a uniform coverage of the sample and a high intensity is satisfied. In addition, at this NA the light impinging on the sample has only a small spread in $k_{\|}$, such that spectral features that are dependent on the incident angle are not washed out.

The diameter of the focal spot was about $15 \mu \mathrm{m}$ and the laser intensity was $20 \mathrm{~mW}$, which results in an intensity of $10 \mathrm{~kW} / \mathrm{cm}^{2}$ on the sample. A 0.3 NA objective lens was used to collect the second-harmonic light at the backside of the sample.

Behind the objective lens, a combination of filters and a prism was used to ensure no fundamental light would reach the detector. The de- 


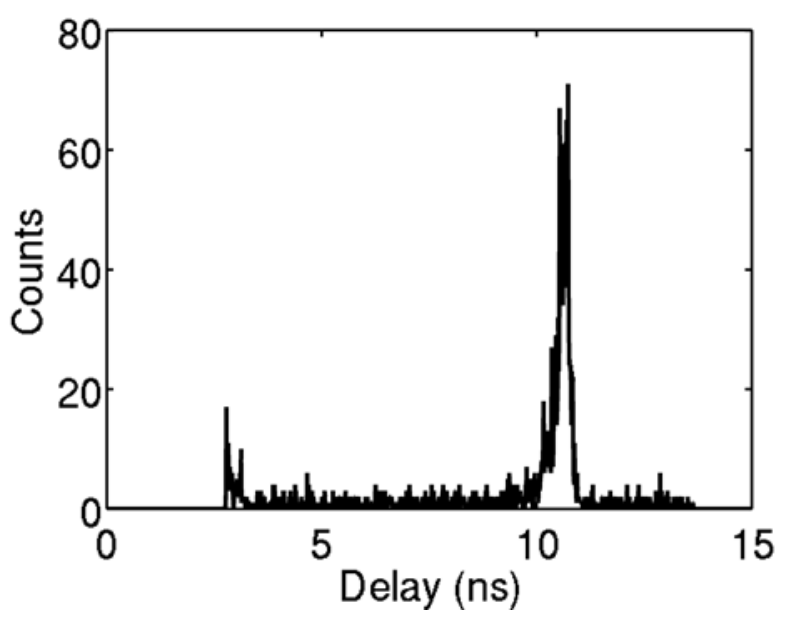

Figure 6.3: Histogram of photon-counts versus delay with reference. The signal always arrives at the same time relative to the reference while the darkcounts are uncorrelated with the laser. Therefore, the amount of measured dark-counts can be reduced by repetition a factor of time/instrument response by only integrating the peak corresponding to the signal and discarding all other counts.

tector was a UV-sensitive PMT (H7421-40, 20\% quantum efficiency at $400 \mathrm{~nm}$ ). Because of the low signal level, the dark-counts of the PMT cause a considerable contribution to the detected signal. To reduce this source of noise, the PMT was coupled to a time-correlated single photon counting system (TCSPC). This system recorded the arrival time of each count, with a resolution of up to $200 \mathrm{ps,} \mathrm{relative} \mathrm{to} \mathrm{a} \mathrm{reference}$ derived from the laser system. Second-harmonic generation is an instantaneous process, therefore the signal counts should coincide with the arrival of the laser pulse at the sample. The TCSPC system recorded a histogram of arrival times for each pulse shape (Figure 6.3). The peak in this Figure corresponds to the signal counts and is shifted in time from zero as a result of different delays of the electronic signals due to the used lengths of cable. The width of the peak is the instrument response function (IRF) of the system. We processed the histogram by integrating the peak and discarding all other counts. In this procedure most dark-counts of the detector are discarded and the number of darkcounts in the signal is reduced by the ratio IRF/(repetition rate).

A chirp scan of the SHG signal was recorded for each sample by applying consecutive quadratic phase profiles on the pulse shaper. For 


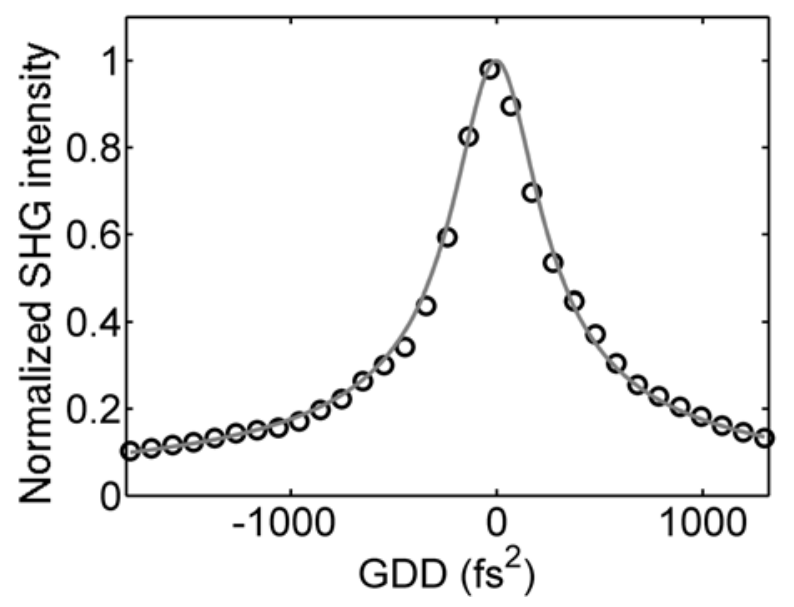

Figure 6.4: Data from a chirp scan of SHG on a BBO-crystal. For the fit, the input pulse is assumed to be the measured laser spectrum without any phase distortion. This assumption lead to a good fit, showing that the input pulse was indeed transform-limited.

each phase profile, the signal on the PMT was accumulated for 30 seconds. The full scan of the chirp was repeated a total of ten times on each structure. The scans on different samples were performed between scans on a BBO crystal. The scans on the BBO crystal were used as a reference in the analysis and to check if the spectral phase of the input pulse remained constant during the experiments.

\subsection{Results}

The average of the scans on the BBO crystal are shown in Figure 6.4. All produced curves were identical within the shot-noise limit, showing that the experimental conditions did not change over the course of the experiment.

The curve was simulated by the method described in Chapter 3, taking into account the measured laser spectrum. The maximum of the scan is not at the point where the zero group delay dispersion was applied on the shaper. Therefore we used the origin as a free parameter in the fit to the data. With the adjusted origin, a good agreement between the simulation and data is found. The good agreement shows that the input pulse had no distortions in the spectral phase beyond 

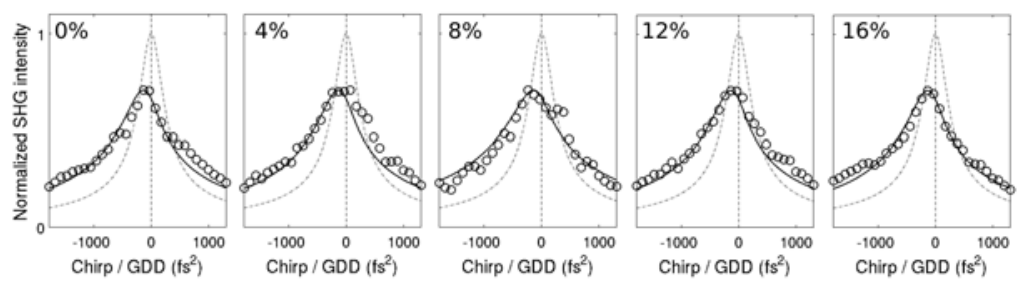

Figure 6.5: Chirp scan data for the five hole arrays with different amounts of disorder. The data from the BBO-crystal is plotted in the background (dashed lines). All the curves show the same features, a broadening and a shift to the left relative to the BBO peak

a slight second order phase, causing the shift of the origin. We will use the shifted maximum of the curve to define the zero group delay dispersion (GDD) point in the analysis of the scans on the hole arrays.

The chirp scans on the hole arrays resulted curve that is distinctly different from the curve on the BBO crystal (Figure 6.5). The curves are much wider and the maximum is shifted to a negative GDD. Such behaviour can indicate the presence of a resonance on the sample (Chapter 3). As the disorder in the placement of the holes was increased, these features did not change. Thus, the disorder, at the level it was introduced in the array, does not seem to influence the process of SHG.

The chirp scan data can be simulated by assuming the presence of a resonance on the sample, as described in Chapter 3. The position, width and amplitude of the resonance are the only fit parameters. Using these parameters an excellent fit to the data is obtained (Figure 6.6). The resonance that fits the data is blue shifted relative to the laser spectrum and very narrow for a resonance on a metal structure (Figure 6.7). The fit parameters are the center position at $791 \pm 3 \mathrm{~nm}$ and a lifetime of $55 \pm 16 \mathrm{fs}$. 


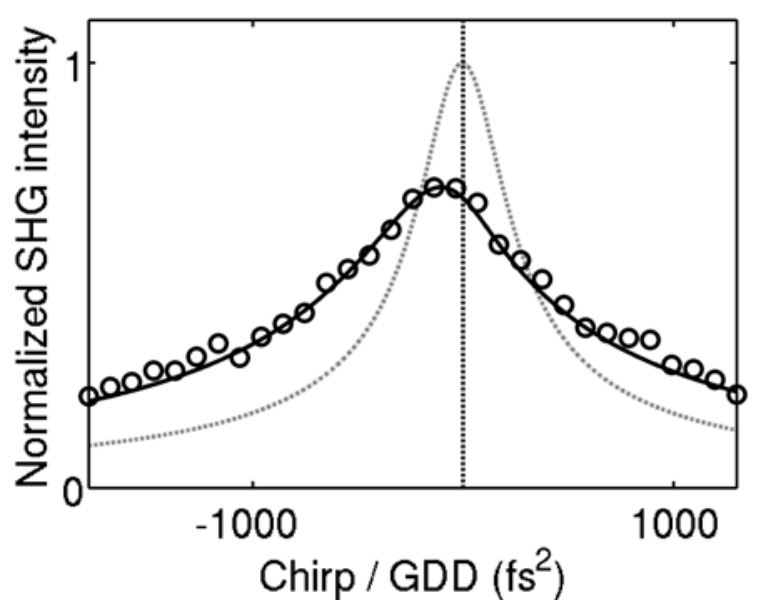

Figure 6.6: Fit to a chirp scan on one hole arrays. The transform-limited input pulse is assumed to be distorted by a resonance on the structure. This assumption results in a good fit to the data. The scan on the hole array was scaled down by $\sqrt{2}$ to better show the difference in center position and width between the scans.

\subsection{Discussion}

The chirp-scan curve on the hole arrays are different from the curve on a BBO crystal. This difference indicates that the SHG is generated in a resonance on the arrays. The disorder that we introduced in the positions of the holes did not influence the shape of the chirp scan and the data from all the arrays can be fit by the same resonance. That spacing of the holes does not change the signature of the SHG process suggests that the resonance is predominantly a property of a single hole.

In a preliminary follow-up experiment, the laser spectrum was shifted to a shorter wavelength and the chirp-scan measurement was performed again. In this measurement the peak of the chirp scan was shifted to a positive GDD relative to a scan on a BBO crystal. This behaviour was expected as now the laser spectrum was blue shifted compared to the position of the resonance at $791 \mathrm{~nm}$. This follow-up experiment provides more evidence for the existence of a long-lived resonance at this position in the spectrum.

The resonance has a long lifetime ( $55 \mathrm{fs}$ ) for a plasmonic structure, as most (particle) plasmon resonances have sub 20 fs lifetimes. The lifetime is also much longer than the transmission time through a hole 


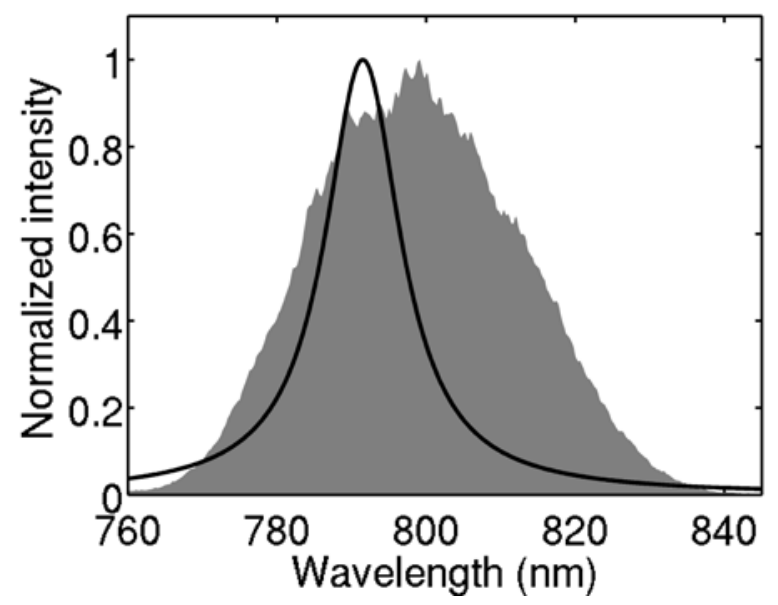

Figure 6.7: The resonance that fits the data in Figure 6.6. The resonance is positioned at the blue side of the laser spectrum (shaded), causing the shift of the maximum to the left, relative to the BBO curve.

array, which is typically $1-4 \mathrm{fs}^{75,80}$. The resonance does not appear as a clear feature in the linear transmission spectrum. Verhagen et al. ${ }^{81}$ investigated the near-field intensity on hole arrays via up-conversion measurements. They found that the same resonances on their structure describe the spectrum of the enhanced near-field and the far-field transmission. This connection does not seem to hold for the resonance behind the SHG on our samples. However, the Q-factor of the resonance is about 10, which means that, due to the enhanced near-field, its contribution to the SHG can be up to 100 times stronger than its contribution to the linear transmission. 


\section{Chapter 7 \\ QUANTUM CONTROL SPECTROSCOPY OF SOLVENT-INDUCED DECOHERENCE}

Coherent control has been recognized as a potential spectroscopic tool for the study of complex molecular systems. With this technique, a molecular system is selectively guided through a potential energy landscape towards a target quantum state by shaped light fields, often employing a closedloop learning algorithm. The central idea is that the pulse shapes corresponding to the highest yield contain spectroscopic information concerning the quantum system, yet often the complexity of these pulses hinders their interpretation. Further, achieving control requires coherence in the quantum system under study. In the condensed phase, coherence is typically lost rapidly due to fluctuating interactions between the solvated molecule and its surrounding environment. In this chapter we show how the amount of attainable control on a molecule in solution changes when the fluctuations of its environment are systematically varied.

A single successful learning curve for optimizing stimulated emission from the molecule is reapplied for a range of solvents with varying viscosity, revealing a striking trend in the yield of an optimization that is correlated directly to the decoherence time. Our results provide clear evidence that the environment limits the leverage of control on a molecular system. 


\subsection{Introduction}

It has been found surprisingly easy to come up with control fields for a wide range of systems ${ }^{82}$, from simple atomic systems to large bio-molecules. These control fields exert their influence even when the system is interacting with a fluctuating environment such as a protein or a solvent in which coherence is in general lost rapidly ${ }^{83}$. However, only in a few isolated cases the goal of deriving fundamental properties of the system and the physical mechanism of control has been achieved ${ }^{84-86}$; most often the pulse shape is too complex to interpret. This obstacle hinders the usefulness of coherent control as a research tool and more progress needs to be made in understanding the basic mechanisms of control and how these mechanisms apply to a variety of molecular systems. An important objective is to find 'rules of thumb' for coherent control, where a given shaped pulse produces a predictable and universal response. A promising route to the realization of this goal is to study a set of systems in which some parameter is systematically varied $^{87}$. Here we take this approach to determine the influence of decoherence caused by the environment. By varying the solvent in which a model quantum system is dissolved, we resolve the impact of the environment on our ability to control the system. Particularly, we study how we can manipulate the yield of stimulated emission from a solvated molecule in a range of solvents, thus elucidating their role in the control.

\subsection{Model system}

The influence of the solvent on the photo-dynamics of a solvated molecule is typically observed via a Stokes shift, in which the energy of the emitting state is lowered relative to the absorption band. The origin of this effect is the modified electron distribution of the excited state, which prompts the solvent molecules to reposition. With the solvent in this new position, the energy of the excited state is lowered, while the energy of the ground state is raised. Thus relaxation from the solvated excited state will emit a red-shifted photon compared to the excitation.

Solvation processes are widely studied using Coumarin dyes ${ }^{88}$, as the electronic structure of these dyes undergoes a strong change when excited. In non-polar solvents the solvation process is more amenable to analysis, because the complicating effects of hydrogen bonding and 


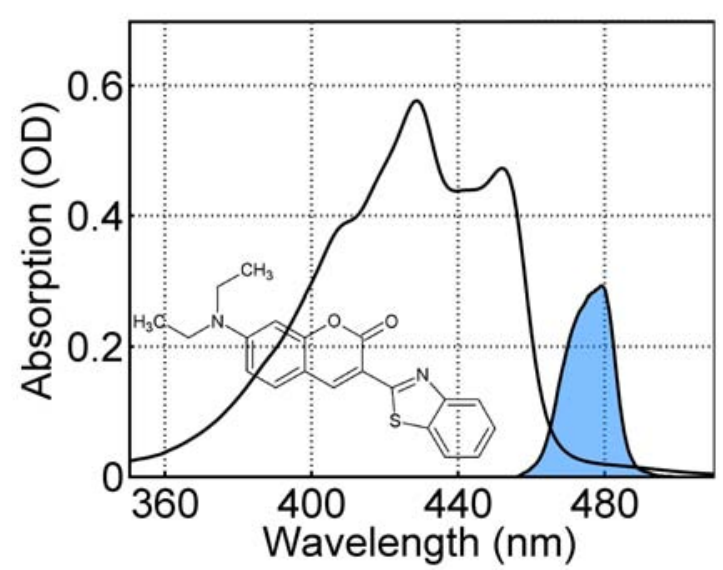

Figure 7.1: Molecular structure of Coumarin 6 and its absorption spectrum in cyclohexane. The laser spectrum (shaded) overlaps with the red wing.

solvent rotation are entirely absent. This simple solvation makes the dynamics of the solvent independent in the 1st $100 \mathrm{fs}$ time window for $\operatorname{control}^{89}$. For these two reasons we have used the laser dye Coumarin 6 dissolved in a range of non-polar solvents to study the effect of solvation on coherent control.

A detailed knowledge of the involved photo-physics is necessary to elucidate the control mechanism. In polar solvents, the amine group of the molecule is in the plane of the rest of the molecule and is resonant with the $\pi$-system. The two groups are joined by a double bond, making the structure rigid. In non-polar solvents, the molecule has lower energy with the bonds around the Nitrogen atom in a pyramidal structure coupled bond to the main molecule via a single bond. This structure results in a somewhat floppy tail ${ }^{90}$. This tail causes the photo-physics to be more complicated. The motion of this floppy tail was found to be involved in a radiationless decay channel that competes with fluorescence ${ }^{91}$. In all non-polar solvents the fluorescence quantum yield is $\sim 0.57$. 


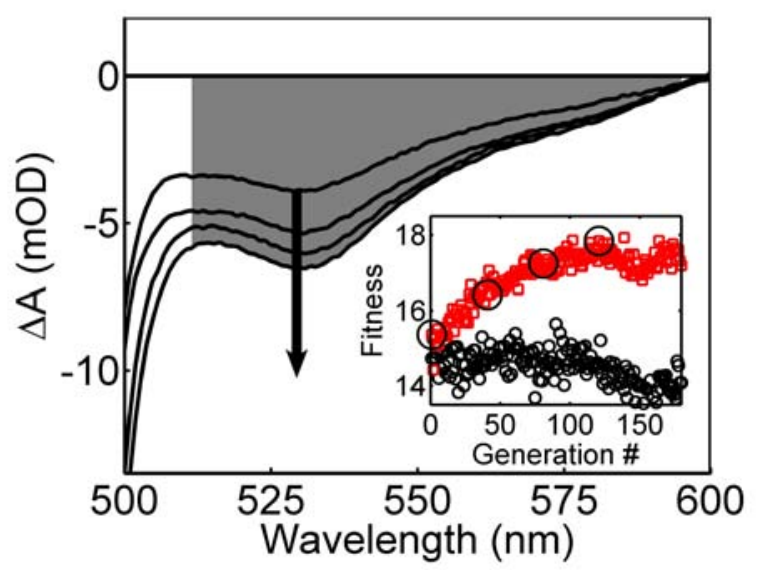

Figure 7.2: Pump-probe spectrum at 1 ns delay in cyclohexane obtained for 4 selected generations (circled) of the learning curve (inset). The inset shows the fitness of the optimal pulse shape (red) and the fitness of the transformlimited pulse (black) for each generation of the optimization. The shaded area indicates the integrated stimulated emission used as a feedback-signal for the optimizations. The arrow shows the increase of this area as a result of control.

\subsection{Optimization}

In an earlier study of coherent control of a solvated laser dye in the weak field limit ${ }^{92}$ it was found that the overlap of the laser spectrum with the red side of the absorption band of the dye is a significant parameter in the control. The importance of this overlap already suggests that the solvent plays a significant role in the control mechanism, as it allows the excitation field to track the change in transition energy in real time as the solvent rearranges. In this study, in contrast to the previous work, we keep the excitation spectrum constant and only modulate the spectral phase. By keeping the amplitude of the excitation spectrum fixed, we make sure that any control achieved will be due to coherences. To be able to address the dye in all states during the solvent relaxation, we pump at the red side of the absorption band and overlap the laser spectrum with the emission band of the dye (Figure 7.1). The optimizations were performed at a moderate laser fluence (1.5 . $10^{15}$ photons $/ \mathrm{cm}^{2}$ ), but because of the low absorption cross-section at the laser wavelength, this fluence is still in the linear absorption regime of the dye (approximately $2 \%$ of the molecules are excited). 


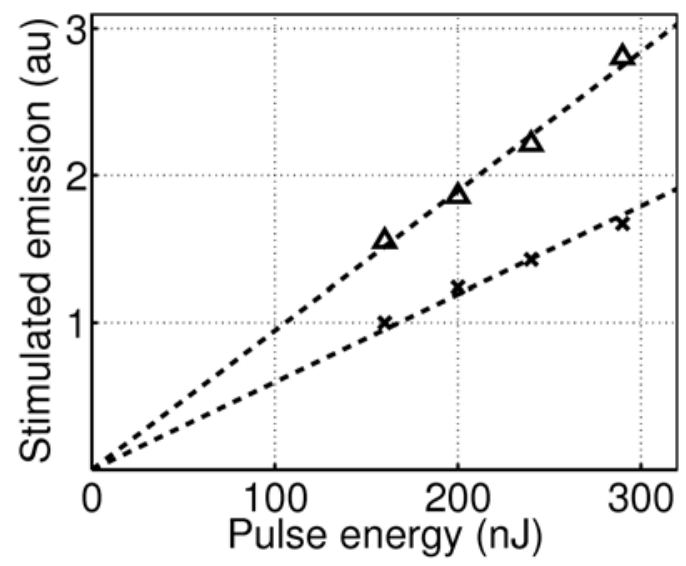

Figure 7.3: Fluence dependence of the fitness value for the transform-limited (crosses) and optimal pulse shape (triangles).

By exciting in the linear absorption regime, trivial control mechanisms based on high-order spectral interference are excluded. The control, if any, will be due to the specific quantum mechanical response of the solvated molecule.

Excitation pulses were passed through a pulse shaper that modulates the spectral phase using a liquid crystal mask. This mask was programmed with phases generated by an evolutionary search algorithm (CMA). Each iteration, the algorithm generated 40 pulse shapes to test in the experiment and used the intensity of stimulated emission as feedback in a learning loop. This feedback signal was derived from a transient absorption spectrum probed $1 \mathrm{~ns}$ after excitation by integration of the gray shaded band in Figure 7.2. Probing at such a long delay ensures complete vibrational relaxation and loss of coherence in the system. In addition, small shifts of the excitation pulse in time will not result in a change of the measured signal, because at this long time delay only the very slow dynamics of the molecule are left over. 


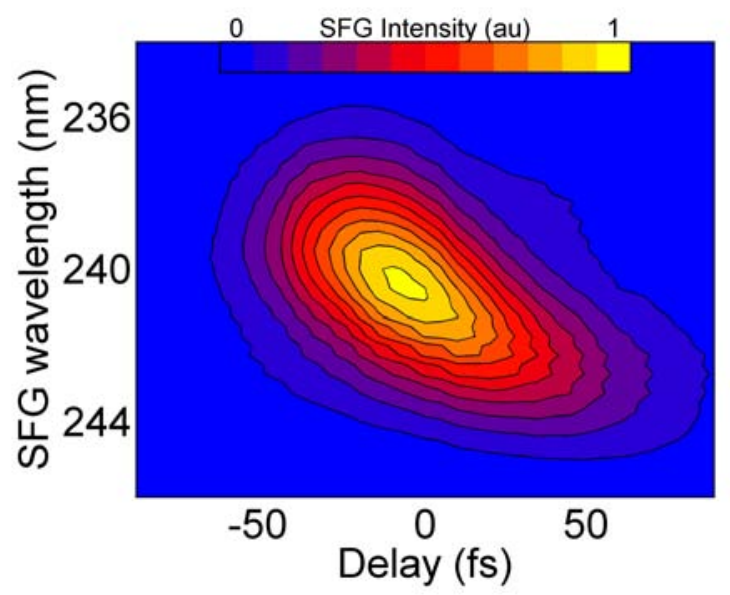

Figure 7.4: A 2-dimensional representation (X-FROG) of the optimal pulse shape found optimization, showing a down-chirp.

\subsection{Results}

The search algorithm was setup to find the pulse shape that maximizes the integrated stimulated emission signal and it typically converged on a solution in $\sim 150$ generations (Figure 7.2 inset). The fluence dependence of the measured stimulated emission is shown in Figure 7.3 both for the transform-limited and the optimal pulse shape. A slight curvature due to saturation effects is seen in the fitness of the transformlimited pulse, but avoidance of this effect alone can not explain the large increase in fitness of the optimal pulse.

\subsubsection{Potential mechanism}

A representation of the optimal pulse shape is shown in Figure 7.4. The pulse is only twice as long as the transform limited pulse and has a down-chirp, i.e. the blue side of the spectrum arrives before the red side. A potential mechanism associated with a down-chirp is wavepacket localization, in which a linear chirp is matched to the anharmonicity of a vibrational mode ${ }^{93}$. However close inspection of the optimal pulse found here reveals a chirp that is significantly nonlinear, i.e. the chirp-rate at the beginning of the pulse (corresponding to a second order spectral phase of $-340 \pm 50 \mathrm{fs}^{2}$ ) is much higher than at the end $\left(-1350 \pm 300 \mathrm{fs}^{2}\right)$. A chirp with such a strong high-order spectral 
dispersion does not result in an optimally focussed wavepacket. An independent optimization, starting from a different random position in the search space, resulted in the same non-linearly chirped pulse, indicating the importance of this feature for the mechanism of control. Futhermore, we could not achieve the same level of control by just using a linearly chirped pulse*. The amount of control attained by the shaped pulse is unprecedented at the excitation fluence we used. In large part the control may be due to the relatively low fluorescence quantum yield of Coumarin 6 in apolar solvents (0.57). This low yield is due to a loss-channel associated with a twisting motion of the amine tail of the molecule ${ }^{94}$. A coherent control pathway that avoids this losschannel, would thereby maximize population in the excited state, inducing a corresponding increase in the stimulated emission signal.

A nonlinear chirp is typical for a frequency-frequency correlation function, suggesting that the mechanism is tracking of the transition frequency. At first sight, such a tracking mechanism may seem counter-intuitive for the optimization of population in the excited state; in the high-power limit a pulse with a down-chirp will in general decrease the population transfer through a pump-dump mechanism by tracking the wave-packet ${ }^{95-97}$. This effect is indeed observed at higher pulse energies when the solvent is replaced by the polar acetone (see Figure 7.8). However, in the weak-field limit, applicable to the apolar solvents, the down-chirped pulse still couples the ground and excited state over the duration of the pulse. By tracking the change in transition energy of the solvated system a coherent interaction can be maintained for the duration of the pulse. Calculations ${ }^{98}$ and experiments ${ }^{99}$ with such chirped pulses show that the result of this coupling is a strong vibrational coherence in the ground state at the expense of coherence in the excited state. Furthermore, when pumping at the red wing of the absorption band, the resulting hot ground state can be excited to an excited state with lower vibrational energy (Figure 7.5). A down-chirp at the red side of the spectrum will in this way slightly increase the population in the excited state while keeping the vibrational excitation of the molecule suppressed. The lower vibrational energy of the excited state may concomitantly limit the amount of internal conversion, because radiationless decay is often coupled to a higher vibrational mode ${ }^{100}$. Hence, suppressing vibration in the excited state will increase the fluorescence quantum

* A scan of the chirp from $-1500 \mathrm{fs}^{2}$ to $+1500 \mathrm{fs}^{2}$ showed a difference of only $4 \%$ in the stimulated emission signal 


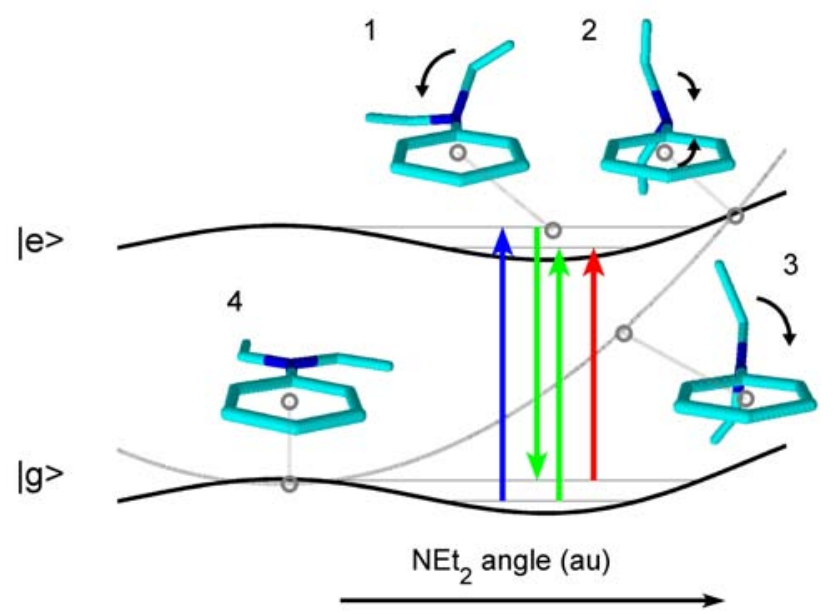

Figure 7.5: Schematic potential energy curves of the planar (dotted) and pyramidal structure (solid). The down-chirped pulse coherently suppresses vibration in the excited state. In Coumarin 6, this vibration can lead to radiationless deactivation by (1) rotation of the amine towards an intersection with the ground state of the twisted planar structure, (2) flipping from the pyramidal to the planar structure (3) and rotation to the energy minimum in this configuration (4).

yield of the system. This type of control may also be applicable to other systems in which vibrational redistribution plays an important role in the photophysics ${ }^{101}$.

The path through the energy landscape corresponding to the radiationless deexcitation mechanism is illustrated schematically in Figure 7.5. In apolar solvents the amine group is in its pyramidal structure instead of the planar structure in polar solvents. In the excited state, the amine group can rotate until a point is reached where the two structures are isoenergetic. At this point, the molecule undergoes a transition to its planar structure and relaxes to the ground state nonradiatively via a rotation of the amine. Coupling of this rotation and a flip-flop motion of the amine tail in Coumarin 120, a closely related Coumarin, which is also aminated at the 7-position, to a fast nonradiative deexcitation process was found by Pal et al. ${ }^{94}$.

The down-chirp on our pulse slightly increases population transfer by excitation from the hot ground state and more importantly suppresses vibration in the excited state, which reduces rotation and/or flipping of the diethylamine tail. By eliminating this loss channel, the 


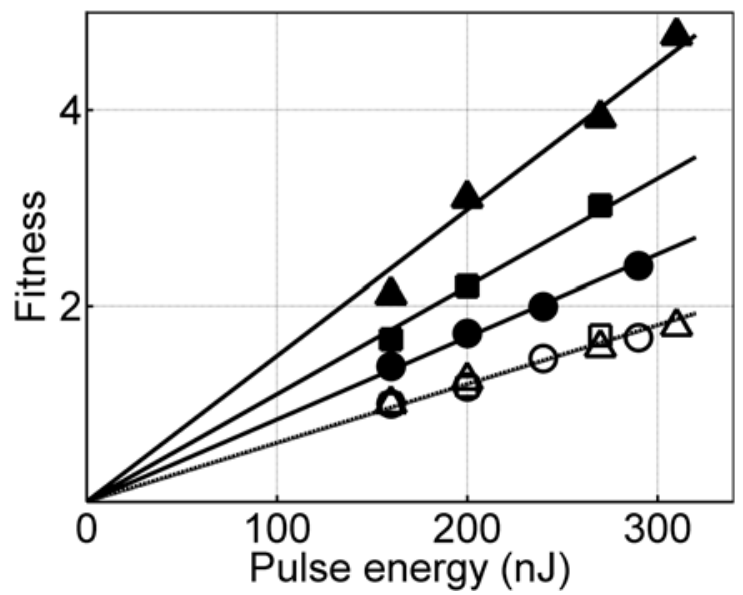

Figure 7.6: Fluence dependence of the fitness for the transform limited (open symbols) and optimal pulse (closed symbols) in the linear alkanes (hexane (triangles), octane (squares) and decane (circles)), showing a linear dependence for both pulse shapes.

excited state population and correspondingly the stimulated emission signal is increased.

In this description of the mechanism we have detailed the branching ratio that was controlled. What acts as a 'probe' and colapses the molecule into the desired state is at present not known. A recent theoretical work by Katz at al. ${ }^{102}$ suggest that the answer may be the solvent itself. In this work the authors have created a system with two excited states that can be controlled with chirped pulses due to coupling to a bath. They find, however, a more complicate dependence on the dephasing time. Initially as the dephasing time is increased, the control yield increases. Then after a maximum, the yield decreases again. The maximum was found at a relatively long dephasing time of $276 \mathrm{fs}$. Our results are expected to fall on the initial rise of the control yield and it is unlikely that a solvent can be found to extend the dephasing time beyond the maximum found. A different model system is required to test the full range of this theory. 


\subsubsection{Solvent dependence}

We determined the effect of the solvent by retracing the search-path to the optimal pulse in one solvent in all other solvents. The best pulse shape in each generation of the original optimization was saved to be used in this retracing experiment. Recording all the pulse shapes in the optimization procedure allows us to check robustness by remeasuring the optimization curve. Additionally we can test the generality of the optimization by changing one or more parameters in the experiment (i.e. laser fluence, solvent). The optimization curve can be viewed as the path followed by the algorithm through the 'fitness landscape', a multi dimensional mountain range of the stimulated emission signal as a function of the separate control parameters. A successful optimization ends at a peak of this mountain range. Variations in the shape of the optimization curve indicate that the fitness landscape is different and thus the underlying physics have changed.

We ran the optimization on Coumarin 6 dissolved in cyclohexane and repeated the measurement using the recorded pulse shapes with the other solvents. The tested solvents were a range of linear alkanes (hexane, octane and decane) and their cyclic counterparts. Repeating the pulse shapes rather than reoptimizing for every solvent avoids the possibility of ending up in a different maximum. Consequently, the optimal results can be compared directly and systematically between the systems. The applicability of repeating was checked by a second successful optimization in octane that converged on the same pulse shape and had the same overall yield as the optimal pulse from the cyclohexane optimization.

The remeasured curves were undersampled by only using the pulse shape from every 8th generation.Undersampling shortens the experimental time, reducing the effect of long-term fluctuations in laser power and additionally allows for a longer integration time, resulting in a clearer picture of the shape of the optimization curve. Multiple runs at varying laser intensities were performed to check the power dependence of the optimization result. The ratio of the stimulated emission signals of the transform-limited and optimal pulse was found to be identical at all laser powers (Figure 7.6), proving that the experiments were carried out in the linear absorption regime.

The curves for the repeated optimizations in the different solvents were normalized to the signal of the transform-limited pulse, allowing for direct comparison (Figure 7.7). The curves were fit with a polynomial function that maps out the ridges and valleys in the traversed 

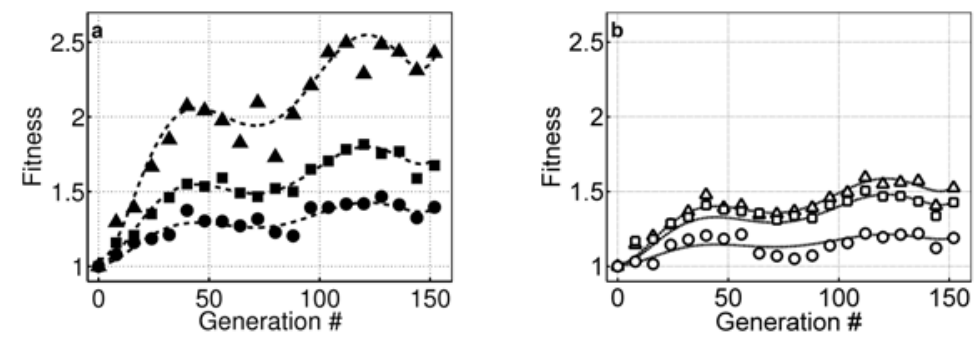

Figure 7.7: Normalized optimization curves for the three linear solvents (a) hexane (triangles), octane (squares) and decane (circles) and the cyclic solvents (b) cyclohexane (triangles), cyclooctane (squares) and cyclodecane (circles). The dashed line in all six curves is the same polynomial function only scaled in amplitude.

fitness landscape. Remarkably, the same polynomial shape fits all curves; only a scaling factor is adjusted. Hence, we conclude that the fitness landscapes of the dye in the different solvents are comparable in topology. The magnitude of the scaling factor shows how well the system can be controlled in the corresponding solvent, with the pulse shape from the cyclohexane optimization. We indeed see that the ability to control the intensity of the stimulated emission strongly depends on the environment.

For comparison the fitness curves where also remeasured in a completely different solvent, namely acetone (Figure 7.8). In this solvent, the absorption is red-shifted causing an increase in the overlap of the laser spectrum with the absorption band. The increased overlap allowed us to reach the non-linear regime in the absorption at pulse energies still linear for the apolar solvents.

The effect of the optimal pulse in the apolar solvents is reversed in acetone. At low pulse energy, the pulse has no significant effect, but as the energy is increased the excitation efficiency is decreased. This effect is expected of a down-chirped pulse at high excitation fluence, because this pulse shape enables a pump-dump process.

In addition to repeating the cyclohexane optimization, we ran an independent optimization in acetone at intermediate pulse energy. The stimulated emission was increased by only a few percent. Furthermore, the optimal pulse was highly fragmented and intangible to further analysis. Therefore we'll analyse only the results in the apolar solvents, in which the observed optimization is linear in pulse energy. 


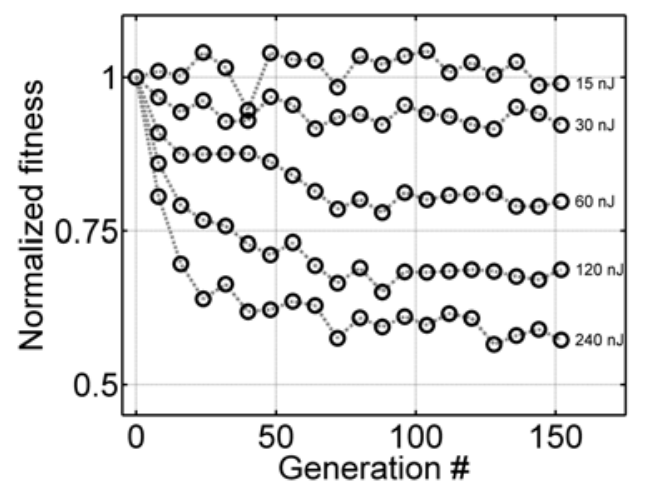

Figure 7.8: Normalized fitness curves of the pulse shapes from the optimization in cyclohexane, remeasured in acetone at varying excitation powers. The stimulated emission is reduced instead of enhanced in this solvent, especially at increasing excitation pulse energy.

The order in which the fitnesses corresponding to the pulse shapes were remeasured does not have a clear physical interpretation, as it depends on the specifics of the search algorithm. Therefore, we plot the normalized fitnesses obtained for each solvent versus the average fitness over all solvents, as measured per pulse shape (Figure 7.9). The points sorted in this way now fall on a straight line, revealing a strong correlation between the fitnesses in the different solvents. From this correlation we conclude that the fitness landscapes of the dye in the different solvents are comparable in topology. The slope of the linear fit is an indication of how well the system can be controlled in the corresponding solvent. The end-point of the linear fits to the data in Figure 7.9 we call the optimization yield. The errors on these values were estimated using a resampling procedure*. The enhancement of the stimulated emission compared to excitation with the transform limited pulse varies from $40 \%$ in decane to $140 \%$ in hexane and from $20 \%$ in cyclodecane to $55 \%$ in cyclohexane.

The fluorescence quantum yield and lifetime of Coumarin 6 is not significantly different in the solvents used. The scaling of the optimization curves is attributed to the different rate at which coherence is lost in the system. Although with coherent control long-lasting coherences

\footnotetext{
* The errors on the slopes of the linear fits were determined via bootstrapping by resampling residuals. The errorbars in Figure 7.10 represent the $2 \sigma$ confidence interval on the end-points of the fit, following from this procedure.
} 


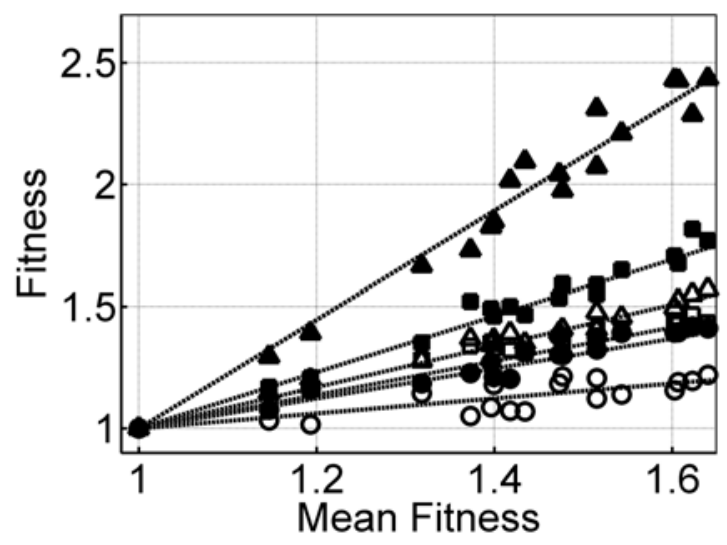

Figure 7.9: The fitness values plotted against the average fitness with the corresponding pulse shapes, showing a strong correlation between the solvents.

can sometimes be found, we assume for simplicity that those coherences will also depend on solvent fluctuations in the same manner as the electronic dephasing time. Taking this limit of fast fluctuations is a reasonable assumption as a large solvent effect is seen whereas the optimal pulse is relatively short, suggesting a short dephasing time. A viscoelastic model ${ }^{103}$, which captures both the inertial and diffusive response of the solvent, predicts that in this limit of fast fluctuations, the dephasing time is inversely proportional to the solvent viscosity ${ }^{104}(\eta)$. When we plot the optimization yield versus the inverse of the viscosity we find a linear dependence for the linear solvent molecules (Figure 7.10). The cyclic solvents have a different vibrational frequency spectrum, which modifies the dephasing time. This difference means that they are not expected to fall on the same line as the linear solvents, but should nonetheless have a similar dependence on viscosity. The optimization yields indeed show this trend. The viscosity dependence of the optimization yield strongly suggests that decoherence is the fundamental limiting factor in the extent of control. 


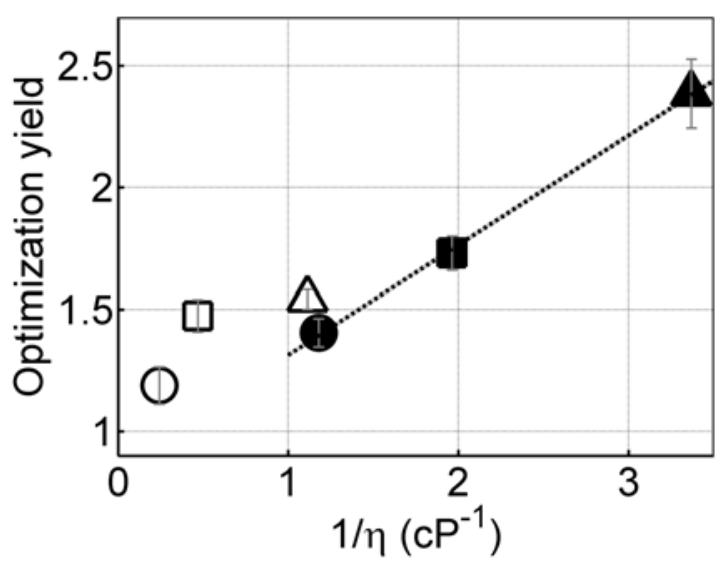

Figure 7.10: The optimization yield relative to the transform limited pulse versus the inverse solvent viscosity $(\eta)$. For the linear solvents the optimization yield is inversely proportional to the viscosity. For the cyclic solvents lower-frequency vibrational modes come into play, but a similar dependence is found.

\subsection{Conclusion}

We have found that, in the weak-field limit, the optimal excitation pulse for Coumarin 6 in non-polar solvents is a down-chirped one. This finding is surprising as down-chirped pulses are generally expected to limit the excitation of the molecule via a pump-dump process. When the solvent was replaced with the polar solvent acetone, the expected minimization of the stimulated emission signal was observed.

We proposed a mechanism for the branching ratio in the control of Coumarin 6 in non-polar solvents, based on coherent suppression of vibration in the excited state, leading to an increased quantum yield. More importantly, by systematically varying the solvent we found that the loss of coherence due to solvent fluctuations, even on the very short time scale of the optimal pulse, is limiting the action of the pulse on the molecule. These results are supported by a recent theoretical study ${ }^{102}$, in which the decoherence by the environment plays an active role in the control mechanism, giving a clue to what act as the 'probe' in weakfield coherent control.

These findings show that a fluctuating environment may be key to coherent control in the weak-field limit. We have found that by engi- 
neering the fluctuations, for example via a judicious choice of solvent, the yield of control may be varied enormously. 
92 Chapter 7. Quantum Control Spectroscopy of Solvent-induced Decoherence 


\section{BibliogRAPHY}

[1] M. Shapiro, J. W. Hepburn, and P. Brumer, Simplified laser control of unimolecular reactions - Simultaneous (omega-1,omega-3) excitation, Chem. Phys. Lett. 149 (1988) 451-454.

[2] R. S. Judson and H. Rabitz, Teaching lasers to control molecules, Phys. Rev. Lett. 68 (1992) 1500-1503.

[3] H. Rabitz, R. de Vivie-Riedle, M. Motzkus, and K. Kompa, Whither the future of controlling quantum phenomena?, Science $\mathbf{2 8 8}$ (2000) 824-828.

[4] N. W. Ashcroft and N. D. Mermin, Solid state physics, Harcourt College Publishers, 1976.

[5] E. Hecht, Optics, Addison-Wesley, 1998.

[6] P. W. Anderson, Absence of diffusion in certain random lattices, Phys. Rev. 109 (1958) 1492-1505.

[7] P. M. Chaikin and T. C. Lubensky, Principles of condensed matter physics, Cambridge University Press, 2000.

[8] S. Mukamel, Principles of nonlinear optical spectroscopy, Oxford University Press, 1995.

[9] P. van der Walle, M. T. W. Milder, L. Kuipers, and J. L. Herek, Quantum control experiment reveals solvation-induced decoherence, Proc. Nat. Acad. Sci. U.S.A 106 (2009) 7714-7717.

[10] P. van der Walle, J. Savolainen, L. Kuipers, and J. L. Herek, Learning from evolutionary optimization by retracing search paths, Chem. Phys. Lett. 483 (2009) 164-167.

[11] N. Hansen and A. Ostermeier, Adapting arbitrary normal mutation distributions in evolution strategies: The covariance matrix adaptation, In Proceedings of the 1996 IEEE International Conference on Evolutionary Computation (1996) 312-317. 
[12] R. Fanciulli, L. Willmes, J. Savolainen, P. Van der Walle, T. Back, and J. L. Herek, Evolution strategies for laser pulse compression, Artificial Evolution (2008) 219-230.

[13] E. Riedle, M. Beutter, S. Lochbrunner, J. Piel, S. Schenkl, S. Sporlein, and W. Zinth, Generation of 10 to $50 \mathrm{fs}$ pulses tunable through all of the visible and the NIR, Appl. Phys. B 71 (2000) 457-465.

[14] J. Savolainen, N. Dijkhuizen, R. Fanciulli, P. A. Liddell, D. Gust, T. A. Moore, A. L. Moore, J. Hauer, T. Buckup, M. Motzkus, and J. L. Herek, Ultrafast energy transfer dynamics of a bioinspired dyad molecule, J. Chem. Phys. B 112 (2008) 2678-2685.

[15] A. M. Weiner, Femtosecond pulse shaping using spatial light modulators, Rev. Sci. Inst. 71 (2000) 1929-1960.

[16] H. Ditlbacher, J. R. Krenn, N. Felidj, B. Lamprecht, G. Schider, M. Salerno, A. Leitner, and F. R. Aussenegg, Fluorescence imaging of surface plasmon fields, Appl. Phys. Lett. 80 (2002) 404406.

[17] M. S. Robbins and B. J. Hadwen, The noise performance of electron multiplying charge-coupled devices, IEEE Trans. on Electron Devices 50 (2003) 1227-1232.

[18] R. Trebino, Frequency-resolved optical gating : The measurement of ultrashort laser pulses, Kluwer Academic, 2002.

[19] P. van der Walle, H. L. Offerhaus, J. L. Herek, and A. Jafarpour, Tailoring a coherent control solution landscape by linear transforms of spectral phase basis, Opt. Express 18 (2010) 973-987.

[20] A. Jafarpour, J. Savolainen, R. de Jong, J. Middag, D. P. Sprunken, P. van der Walle, D. Yang, and J. L. Herek, Robust orthogonal parameterization of evolution strategy for adaptive laser pulse shaping, Opt. Express 17 (2009) 11986-12000.

[21] O. M. Shir, Niching in derandomized evolution strategies and its applications in quantum control, Ph.D. thesis, Leiden University, 2008.

[22] J. C. M. Diels, J. J. Fontaine, I. C. McMichael, and F. Simoni, Control and measurement of ultrashort pulse shapes (in amplitude and phase) with femtosecond accuracy, Appl. Opt. 24 (1985) 1270-1282. 
[23] B. Lamprecht, J. R. Krenn, A. Leitner, and F. R. Aussenegg, Resonant and off-resonant light-driven plasmons in metal nanoparticles studied by femtosecond-resolution third-harmonic generation, Phys. Rev. Lett. 83 (1999) 4421-4424.

[24] B. Lamprecht, J. R. Krenn, A. Leitner, and F. R. Aussenegg, Particle-plasmon decay-time determination by measuring the optical near-field's autocorrelation: Influence of inhomogeneous line broadening, Appl. Phys. B 69 (1999) 223-227.

[25] B. Lamprecht, A. Leitner, and F. R. Aussenegg, SHG studies of plasmon dephasing in nanoparticles, Appl. Phys. B 68 (1999) 419-423.

[26] M. W. Klein, T. Tritschler, M. Wegener, and S. Linden, Lineshape of harmonic generation by metallic nanoparticles and metallic photonic crystal slabs, Phys. Rev. B 72 (2005).

[27] I. I. Rabi, Space quantization in a gyrating magnetic field, Phys. Rev. 51 (1937) 652.

[28] N. Dudovich, B. Dayan, S. M. Gallagher Faeder, and Y. Silberberg, Transform-limited pulses are not optimal for resonant multiphoton transitions, Phys. Rev. Lett. 86 (2001) 47-50.

[29] T. Vartanyan, M. Simon, and F. Trager, Femtosecond optical second harmonic generation by metal clusters: The influence of inhomogeneous line broadening on the dephasing time of surface plasmon excitation, Appl.Phys. B 68 (1999) 425-431.

[30] L. Novotny and B. Hecht, Principles of nano-optics, Cambridge University Press, 1996.

[31] P. Vasa, C. Ropers, R. Pomraenke, and C. Lienau, Ultra-fast nanooptics, Laser \& Photon. Rev. 3 (2009) 483-507.

[32] J. P. Clerc, G. Giraud, and J. M. Luck, The AC electricalconductivity of binary disordered-systems, percolation clusters, fractals and related models, Adv. Phys. 39 (1990) 191-308.

[33] D. J. Bergman and D. Stroud, Physical-properties of macroscopically inhomogeneous-media, Solid State Phys. 46 (1992) 147-269.

[34] L. Zekri, R. Bouamrane, and N. Zekri, New method for the exact determination of the effective conductivity and the local field in RLC networks, J. Phys. A 33 (2000) 649-656. 
[35] A. N. Lagarkov, K. N. Rozanov, A. K. Sarychev, and N. A. Simonov, Experimental and theoretical study of metal-dielectric percolating films at microwaves, Physica A 241 (1997) 199-206.

[36] D. A. Genov, A. K. Sarychev, and V. M. Shalaev, Plasmon localization and local field distribution in metal-dielectric films, Phys. Rev. E 67 (2003) 056611.

[37] U. Kreibig and M. Vollmer, Optical properties of metal clusters, Springer-Verlang, 1995.

[38] P. B. Johnson and R. W. Cristy, Optical constants of the nobel metals, Phys. Rev. B 6 (1972) 4370-4379.

[39] P. Drude, Zur elektronentheorie der metalle, Annalen der Physik 306 (1900) 566.

[40] A. K. Sarychev and V. M. Shalaev, Electromagnetic field fluctuations and optical nonlinearities in metal-dielectric composites, Phys. Rep. 335 (2000) 276-371.

[41] A. Aharony, R. Blumenfeld, and A. Brooks Harris, Distribition of the logarithms of currents in percolating risistor networks. I. Theory, Phys. Rev. B 47 (1993) 5756-5769.

[42] X. T. Li and M. I. Stockman, Highly efficient spatiotemporal coherent control in nanoplasmonics on a nanometer-femtosecond scale by time reversal, Phys. Rev. B 77 (2008).

[43] P. Tuchscherer, C. Rewitz, D. V. Voronine, F. J. Garcia de Abajo, W. Pfeiffer, and T. Brixner, Analytic coherent control of plasmon propagation in nanostructures, Opt. Express 17 (2009) 1423514259.

[44] M. Moskovits, Surface-enhanced spectroscopy, Rev. Mod. Phys. 57 (2001) 783.

[45] K. Kneipp, H. Kneipp, I. Itzkan, R. R. Dasari, and M. S. Feld, Ultrasensitive chemical analysis by Raman spectroscopy, Chem. Rev. 99 (1999) 2957.

[46] C. Y. Chen and E. Burstein, Giant Raman scattering by molecules at metal-island films, Phys. Rev. Lett. 45 (1980) 1287. 
[47] P. F. Liao, J. G. Bergman, D. S. Chemla, A. Wokaun, J. Melngailis, A. M. Howryluk, and N. P. Economou, Surfase-enhanced Raman scattering from microlithographic silver particle surfaces, Chem. Phys. Lett. 82 (1981) 355.

[48] A. Wei, B. Kim, B. Sadtler, and S. L. Tripp, Tunable surfaceenhanced Raman scattering from large gold nanoparticle arrays, ChemPhysChem 2 (2001) 743.

[49] L. Gunnarsson, E. J. Bjerneld, H. Xu, S. Petronis, B. Kasemo, and M. Kall, Interparticle coupling effects in nanofabricated substrates for surface-enhanced Raman scattering, Appl. Phys. Lett. 78 (2001) 802-804.

[50] N. Felidj, J. Aubard, G. Levi, J. R. Krenn, A. Hohenau, G. Schider, A. Leitner, and F. R. Aussenegg, Optimized surface-enhanced Raman scattering on gold nanoparticle arrays, Appl. Phys. Lett. 82 (2003) 3095-3097.

[51] M. I. Stockman, D. J. Bergman, and T. Kobayashi, Coherent control of nanoscale localization of ultrafast optical excitation in nanosystems, Phys. Rev. B 69 (2004).

[52] J. M. Gunn, M. Ewald, and M. Dantus, Polarization and phase control of remote surface-plasmon-mediated two-photon-induced emission and waveguiding, Nano Lett. 6 (2006) 2804-2809.

[53] M. Durach, A. Rusina, and M. I. Stockman, Toward full spatiotemporal control on the nanoscale, Nano Lett. 7 (2007) 31453149.

[54] T. Brixner, F. J. Garcia de Abajo, J. Schneider, and W. Pfeiffer, Nanoscopic ultrafast space-time-resolved spectroscopy, Phys. Rev. Lett. 95 (2005) 093901.

[55] J. Lehmann, M. Merschdorf, W. Pfeiffer, A. Thon, S. Voll, and G. Gerber, Surface plasmon dynamics in silver nanoparticles studied by femtosecond time-resolved photoemission, Phys. Rev. Lett. 85 (2000) 2921-2924.

[56] M. I. Stockman and P. Hewageegana, Nanolocalized nonlinear electron photoemission under coherent control, Nano Lett. 5 (2005) 2325-2329. 
[57] A. Kubo, K. Onda, H. Petek, Z. J. Sun, Y. S. Jung, and H. K. Kim, Femtosecond imaging of surface plasmon dynamics in a nanostructured silver film, Nano Lett. 5 (2005) 1123-1127.

[58] M. Aeschlimann, M. Bauer, D. Bayer, T. Brixner, F. J. de Abajo, W. Pfeiffer, M. Rohmer, C. Spindler, and F. Steeb, Adaptive subwavelength control of nano-optical fields, Nature 446 (2007) 301304.

[59] V. M. Shalaev and A. K. Sarychev, Nonlinear optics of random metal-dielectric films, Phys. Rev. B 57 (1998) 13265-13288.

[60] T. Baumert, T. Brixner, V. Seyfried, M. Strehle, and G. Gerber, Femtosecond pulse shaping by an evolutionary algorithm with feedback, Appl. Phys. B 65 (1997) 779-782.

[61] Y. Fang, N. H. Seong, and D. D. Dlott, Measurement of the distribution of site enhancements in surface-enhanced Raman scattering, Science 321 (2008) 388-392.

[62] M. Kretschmann and A. A. Maradudin, Band structures of twodimensional surface-plasmon polaritonic crystals, Phys. Rev. B 66 (2002).

[63] C. Ropers, D. J. Park, G. Stibenz, G. Steinmeyer, J. Kim, D. S. Kim, and C. Lienau, Femtosecond light transmission and subradiant damping in plasmonic crystals, Phys. Rev. Lett. 94 (2005).

[64] T. W. Ebbesen, H. J. Lezec, H. F. Ghaemi, T. Thio, and P. A. Wolff, Extraordinary optical transmission through sub-wavelength hole arrays, Nature 391 (1998) 667-669.

[65] H. A. Bethe, Theory of diffraction by small holes, Phys. Rev. 66 (1944) 163-182.

[66] H. F. Ghaemi, T. Thio, D. E. Grupp, T. W. Ebbesen, and H. J. Lezec, Surface plasmons enhance optical transmission through subwavelength holes, Phys. Rev. B 8 (1998) 67796782.

[67] K. L. van der Molen, F. B. Segerink, N. F. van Hulst, and L. Kuipers, Influence of hole size on the extraordinary transmission through subwavelength hole arrays, Appl. Phys. Lett. 85 (2004) 4316-4318. 
[68] K. J. Klein Koerkamp, S. Enoch, F. B. Segerink, N. F. van Hulst, and L. Kuipers, Strong influence of hole shape on extraordinary transmission through periodic arrays of subwavelength holes, Phys. Rev. Lett. 92 (2004).

[69] S. T. Chase and R. D. Joseph, Resonant array bandpass filters for the far infrared, Appl. Opt. 22 (1983) 1775-1779.

[70] C. Genet and T. W. Ebbesen, Light in tiny holes, Nature 445 (2007) 39-46.

[71] F. J. Garcia de Abajo, Colloquium: Light scattering by particle and hole arrays, Rev. Mod. Phys. 79 (2007) 1267-1290.

[72] M. Airola, Y. Liu, and S. Blair, Second-harmonic generation from an array of sub-wavelength metal apertures, J. Opt. A 7 (2005) S118-S123.

[73] W. Fan, S. Zhang, N. C. Panoiu, A. Abdenour, S. Krishna, R. M. Osgood, K. J. Malloy, and S. R. J. Brueck, Second harmonic generation from a nanopatterned isotropic nonlinear material, Nano Lett. 6 (2006) 1027-1030.

[74] J. A. H. van Nieuwstadt, M. Sandtke, R. H. Harmsen, F. B. Segerink, J. C. Prangsma, S. Enoch, and L. Kuipers, Strong modification of the nonlinear optical response of metallic subwavelength hole arrays, Phys. Rev. Lett. 97 (2006) 146102.

[75] J. C. Prangsma, D. van Oosten, R. J. Moerland, and L. Kuipers, Increase of group delay and nonlinear effects with hole shape in subwavelength hole arrays, New J. Phys. 12 (2010) 013005.

[76] R. W. Woods, Anomalous diffraction gratings, Phys. Rev. 48 (1935) 928936.

[77] U. Fano, Effects of configuration interaction on intensities and phase shifts, Phys. Rev. 124 (1961) 1866-1878.

[78] M. Sarrazin, J. P. Vigneron, and J. M. Vigoureux, Role of wood anomalies in optical properties of thin metallic films with a bidimensional array of subwavelength holes, Phys. Rev. B 67 (2003).

[79] C. Genet, M. P. van Exter, and J. P. Woerdman, Fano-type interpretation of red shifts and red tails in hole array transmission spectra, Opt. Comm. 225 (2003) 331-336. 
[80] R. Muller, C. Ropers, and C. Lienau, Femtosecond light pulse propagation through metallic nanohole arrays: The role of the dielectric substrate, Opt. Express 12 (2004) 5067-5081.

[81] E. Verhagen, L. Kuipers, and A. Polman, Field enhancement in metallic subwavelength aperture arrays probed by erbium upconversion luminescence, Opt. Express 17 (2009) 14586-14598.

[82] H. A. Rabitz, M. M. Hsieh, and C. M. Rosenthal, Quantum optimally controlled transition landscapes, Science 303 (2004) 19982001.

[83] T. Brixner, N. H. Damrauer, P. Niklaus, and G. Gerber, Photoselective adaptive femtosecond quantum control in the liquid phase, Nature 414 (2001) 57-60.

[84] C. Daniel, J. Full, L. Gonzalez, C. Lupulescu, J. Manz, A. Merli, S. Vajda, and L. Woste, Deciphering the reaction dynamics underlying optimal control laser fields, Science 299 (2003) 536-539.

[85] J. L. Herek, W. Wohlleben, R. J. Cogdell, D. Zeidler, and M. Motzkus, Quantum control of energy flow in light harvesting, Nature 417 (2002) 533-535.

[86] T. C. Weinacht, J. Ahn, and P. H. Bucksbaum, Controlling the shape of a quantum wavefunction, Nature 397 (1999) 233-235.

[87] D. Cardoza, M. Baertschy, and T. Weinacht, Understanding learning control of molecular fragmentation, Chem. Phys. Lett. 411 (2005) 311-315.

[88] C. Reichardt, Solvatochromic dyes as solvent polarity indicators, Chem. Rev. 94 (1994) 2319-2358.

[89] J. C. Gumy, O. Nicolet, and E. Vauthey, Investigation of the solvation dynamics of an organic dye in polar solvents using the femtosecond transient grating technique, J. Phys. Chem. A 103 (1999) 10737-10743.

[90] A. K. Satpati, M. Kumbhakar, D. K. Maity, and H. Pal, Photophysical investigations of the solvent polarity effect on the properties of Coumarin-6 dye, Chem. Phys. Lett. 407 (2005) 114-118. 
[91] G. Jones II, W.R. Jackson, C. Choi, and W.R. Bergmark, Solvent effects on emission yield and lifetime for coumarin laser dyes: Requirements for a rotatory decay mechanism, J. Phys. Chem. 89 (1985) 294-300.

[92] V. I. Prokhorenko, A. M. Nagy, and R. J. D. Miller, Coherent control of the population transfer in complex solvated molecules at weak excitation. An experimental study, J. Chem. Phys. 122 (2005) 184502.

[93] S. H. Lee, K. H. Jung, J. H. Sung, K. H. Hong, and C. H. Nam, Adaptive quantum control of DCM fluorescence in the liquid phase, J. Chem. Phys. 117 (2002) 9858-9861.

[94] H. Pal, S. Nad, and M. Kumbhakar, Photophysical properties of Coumarin-120: Unusual behavior in nonpolar solvents, J. Chem. Phys. 119 (2003) 443-452.

[95] C. J. Bardeen, V. V. Yakovlev, J. A. Squier, and K. R. Wilson, Quantum control of population transfer in green fluorescent protein by using chirped femtosecond pulses, J. Am. Chem. Soc. 120 (1998) 13023-13027.

[96] J. S. Cao, C. J. Bardeen, and K. R. Wilson, Molecular pi pulses: Population inversion with positively chirped short pulses, J. Chem. Phys. 113 (2000) 1898-1909.

[97] O. Nahmias, O. Bismuth, O. Shoshana, and S. Ruhman, Tracking excited state dynamics with coherent control: Automated limiting of population transfer in LDS750, J. Phys. Chem. A 109 (2005) 8246-8253.

[98] S. Ruhman and R. Kosloff, Application of chirped ultrashort pulses for generating large-amplitude ground-state vibrational coherence - A computer-simulation, J. Opt. Soc. Am. B 7 (1990) 17481752.

[99] C. J. Bardeen, Q. Wang, and C. V. Shank, Selective excitation of vibrational wave-packet motion using chirped pulses, Phys. Rev. Lett. 75 (1995) 3410-3413.

[100] S. H. Lin, Rate of interconversion of electronic and vibrational energy, J. Chem. Phys. 44 (1966) 3759-3767. 
[101] K. Ishii, S. Takeuchi, and T. Tahara, A 40-fs time-resolved absorption study on cis-stilbene in solution: Observation of wavepacket motion on the reactive excited state, Chem. Phys. Lett. 398 (2004) 400-406.

[102] G. Katz, M. A. Ratner, and R. Kosloff, Control by decoherence: weak field control of an excited state objective, New J. Phys. 12 (2010) 015003.

[103] M. Berg, Viscoelastic continuum model of nonpolar solvation. 1. Implications for multiple time scales in liquid dynamics, J. Phys. Chem. A 102 (1998) 17-30.

[104] M. A. Berg, K. D. Rector, and M. D. Fayer, Two-pulse echo experiments in the spectral diffusion regime, J. Chem. Phys. 113 (2000) 3233-3242. 


\section{SUMMARY}

This thesis is about coherent control on disordered samples. Coherent control is the steering of processes with light of different frequencies. The relative phases between the frequencies determines in which direction the process is steered. When many frequencies are used, the light composes a short pulse. Changing the relative phases of the frequencies changes the shape of this pulse. We used such shaped pulses to guide processes on our samples. Pulse shaping and the different disordered samples are introduced in chapter 1, after which chapter 2 proceeds with the experimental details behind our studies.

To perform a measurement with a shaped pulse, a nonlinear process is required. Second-harmonic generation (SHG) is one of the most straightforward nonlinear processes and plays an important role in this thesis. In chapter 3 theory of SHG is developed. Specifically, we determine how to calculate the SHG intensity from shaped pulses on samples with a resonance near the frequency of the illuminating light. We show that two mechanisms that broaden the line width of a resonance can not be distinguished by measuring the SHG signal. In the absence of resonances the maximum SHG intensity is produced by a transformlimited pulse. This pulse shape is required for many nonlinear optical spectroscopies and maximizing the SHG intensity is a useful method to obtain it. For such optimizations, algorithms are used that tune the coefficients for basis functions of the spectral phase. We looked at the fitness landscape of the SHG when a common basis set is used and by inspecting projections of the landscape we constructed a more efficient basis set, that will make optimizations of SHG more efficient.

In chapter 4 we describe simulations of the electric field in a random electrical network. The electrical network serves as a model for a film of randomly positioned gold islands, which we studied as reported in chapter 5 . The simulations show that a metal density close to the percolation threshold results in a structure that has favourable properties for selective local excitation of the surface.

In chapter 5 we proceed with this experiment on a random gold structure. The structure was coated with a layer of quantum dots. We 
imaged the fluorescence from this layer with a microscope and in this way were able to detect the local field intensities on the surface. We found that some control on the field intensity is possible, but the effect is minimal compared to the results of the simulations. The reason for this discrepancy is in the radiative damping of the oscillations, which was not included in the simulation. The radiative damping of plasmons causes dephasing within $\sim 20$ fs, making control with shaped pulses difficult.

We further studied SHG from arrays of sub-wavelength holes in a gold film. The results of this study are presented in chapter 6 . The amount of disorder in the positions of the holes in the arrays was varied. We found that the the SHG is produced in a resonance with a long lifetime of $55 \mathrm{fs}$. This lifetime is longer than most plasmonic resonances and shows that the effect of radiative damping is decreased on this structure. Furthermore, the SHG from all the structures has the same features. The disorder does not influence the SHG and the same resonance was found on all structures. Thus, it is likely that the resonance is a property of a single hole.

Finally, we studied the coherent control of a dye in solution. Here the disorder is not in the structure, but in the transition frequency of the dye. Due to the interaction of the dye with the solvent, this frequency is constantly changing. These changes cause coherence to be lost in the system in a short time. We ran a closed-loop optimization to maximize the stimulated emission from the dye. All the pulse shapes tried in the optimization were saved and subsequently tried with the dye dissolved in other solvents. We found that the yield of the optimization varied greatly with the used solvent. These results formed a trend with the viscosity of the particular solvents. The viscosity can be related directly to the dephasing time, the time in which coherence is lost in the system. The trend of the optimization yield versus the viscosity shows directly that the fluctuations of the solvent are limiting the coherent control of the dye. 


\section{SAMENVATting}

Dit proefschrift gaat over coherente sturing op wanordelijke samples. Coherente sturing is het besturen van processen met licht van verschillende frequenties. De relatieve fase tussen de frequenties bepaald in welke richting het proces gestuurd wordt. Als er veel frequenties gebruikt worden, dan vormt het licht een korte puls. Door de relatieve fase tussen de frequenties in de puls te veranderen wordt de vorm van de puls beinvloed. Wij hebben zulke vervormde pulsen gebruikt om processen op onze samples te besturen. Het vervormen van pulsen en de verschillende wanordelijke samples worden geintroduceerd in hoofdstuk 1, waarna in hoofdstuk 2 de details van de experimenten volgen.

Om een meting met een gevormde puls te kunnen doen, is een nietlineair proces nodig. Tweede-harmonische generatie (SHG) is een eenvoudig niet-lineair proces and speelt een grote rol in dit proefschrift. In hoofdstuk 3 wordt theorie van SHG ontwikkeld. Specifiek, bepalen we hoe we de SHG intensiteit van gevormde pulsen kunnen berekenen als ze gebruikt worden op samples met een resonantie dichtbij de frequentie van het gebruikte licht. We laten zien dat twee processen die een resonantie verbreden, niet te onderscheiden zijn door middel van metingen van SHG. Als er geen resonanties zijn, dan geeft een transformatie-gelimiteerde puls de maximale SHG intensiteit. Deze puls-vorm is nodig voor vele niet-lineair optische spectroscopien en de maximalisatie van de SHG intensiteit is een goede manier om deze puls te vinden. Bij zulke optimalisaties wordt een algoritme gebruikt dat de coefficienten voor basis functies van de spectrale fase beinvloed. Wij hebben het fitness landschap van SHG bekeken, wanneer een veel gebruikte basis set wordt toegepast. Door projecties van het landschap te bestuderen hebben we een nieuwe basis set kunnen construeren dat de optimalisatie van SHG efficienter maakt.

In hoofdstuk 4 beschrijven we simulaties van het elektrisch veld in wanordelijk elektrisch netwerk. Dit netwerk is een model voor een laag met daarin goud deeltjes op random posities geplaatst. De simulaties laten zien dat wanneer de dichtheid van het metaal de percolatie grens 
bereikt, de structuur de beste eigenschappen heeft om selectief geexciteerd te kunnen worden.

In hoofdstuk 5 gaan we verder met het experiment op de random goud-laag. Een laag van quantum dots werd op het sample gelegd. De fluorescentie van de quantum dots werd door middel van een microscoop afgebeeld waardoor we de locale elektrische velden op het sample konden zien. De experimenten wezen uit dat er enige mate van controle over deze velden mogelijk is, maar dat vergeleken met de simulaties dit effect minimaal is. Dit verschil komt door de radiatieve demping van de oscillaties op het sample, die niet in de simulaties waren meegenomen. De radiatieve demping zorgt ervoor dat oscillaties binnen 20 fs uitdempen, wat sturing met gevormde pulsen moeilijk maakt.

Verder bestudeerden we SHG van roosters met kleine gaatjes in een gouden film. De resultaten hiervan staan in hoofdstuk 6. De hoeveelheid wanorde in de posities van de gaten werd gevarieerd. We ontdekten dat de SHG geproduceerd wordt in een resonantie met een lange levensduur, $55 \mathrm{fs}$. Deze levensduur is langer dan de meeste plasmonische resonanties en geeft aan dat radiatieve demping op deze structuur verminderd is. Verder zag het SHG signaal van alle structuren er hetzelfde uit. De wanorde had geen invloed en dezelfde resonantie werd op elke structuur gevonden. Hieruit blijkt dat de resonantie waarschijnlijk voortkomt uit de eigenschappen van individuele gaten.

Als laatste bestudeerden we de coherente sturing van een kleurstofmolecuul in een oplosmiddel. In dit sample zit de wanorde niet in de structuur, maar in de transitie frequentie van het molecuul. Door de interactie van het molecuul met het oplosmiddel, is deze frequentie voortdurend anders. Deze veranderingen zorgen ervoor dat de coherentie in het systeem snel verloren gaat. We deden een optimalisatie om de gestimuleerde emissie van het molecuul zo groot mogelijk te maken. Alle pulsvormen die in de optimalisatie gebruikt werden, werden opgeslagen. Vervolgens werden de pulsen opnieuw geprobeerd, maar nu met het molecuul in een ander oplosmiddel. Het bleek dat de verbetering die de optimalisatie opleverde sterk afhing van het gebruikte oplosmiddel. Onze resultaten vormen een trend met de viscositeit van de oplosmiddelen. De viscositeit kunnen we direct relateren aan de defaserings tijd, de tijd waarin de coherentie verloren gaat. Dus, de trend van de optimalisaties met de viscositeit laat direct zien dat de fluctuaties van het oplosmiddel de coherente sturing limiteren. 


\section{ACKNOWLEDGEMENTS}

For many people this will be the first line they read in this thesis. If those people find the time to also browse the rest of this book, they will discover a great variety of ideas and experiments.

The cause of this variety is the combination of my two supervisors, Kobus and Jennifer. The goal of my research was to apply the techniques of Jennifer (coherent control) on the samples of Kobus (nanostructures). In the end, I think, we reached this goal, but on the way there were many interesting questions that tempted me away from the direct route. Jennifer and Kobus, thank you for trusting in me and letting me find my on way.

One of the distractions was the 'Works Council', of which I was a member for two years. During these years I learned a great deal about how science or just any organisation is managed. Miranda, Niels, Roland, Niels and Kim, you were a great team.

My experiments began at Amolf in the lab of the BiMoCo group. The people in this group, Janne, Riccardo, Niels and later Maaike and Puck, made that the work enjoyable, and were always available for help or just a chat. For the technically challenging experiments a great deal of support was needed. It is amazing how many sudden technical difficulties one can run into that make an experiment not work. Hinco, Rob, Ilja and the people at E\&I always had the time to fix these problems or to quickly make something that I needed already yesterday.

Also outside of the BiMoCo group many people contributed to the pleasant atmosphere at Amolf. Especially the BaBo groups, who kind of adopted me after the demise of the BiMoCo group, played a big part in my life at Amolf. I saw too many of you come and go to name all of you, but you know who you are. Thanks guys, for the interesting discussions at lunch, the parties and outings, and for just being there for me to have someone to talk to.

When Jennifer left to Twente and took the lab with her, some measurement still had to be done. So, I had to come to Enschede and do the experiments in the new Optical Sciences group of Jennifer. The circumstances and atmosphere in this group reminded me of Amolf, the 
excellent technical support by Frans and Jeroen, and a friendly interaction between the people. It pleases me that the work that was started during my $\mathrm{PhD}$ will continue in this group. This project will no doubt be successful and I wish Dan and his future co-workers on the project the best of luck.

Finally, a big thanks to Maria for staying with me during the writing of this book. The writing did not come easy for me, but you, having experienced it not long ago yourself, managed to motivate me to move on. Now that we have both completed this phase in our lives, I look forward to our new adventures together in the real world. 

